University of Tennessee Health Science Center

UTHSC Digital Commons

\title{
$5-2011$
}

\section{Emergency Physicians' Perspectives on the Usability of Health Information Exchange}

Shirley A. Thorn

University of Tennessee Health Science Center

Follow this and additional works at: https://dc.uthsc.edu/dissertations

Part of the Emergency Medicine Commons, and the Health and Medical Administration Commons

\section{Recommended Citation}

Thorn, Shirley A. , "Emergency Physicians' Perspectives on the Usability of Health Information Exchange" (2011). Theses and Dissertations (ETD). Paper 275. http://dx.doi.org/10.21007/etd.cghs.2011.0318. 


\title{
Emergency Physicians' Perspectives on the Usability of Health Information Exchange
}

\begin{abstract}
Emergency physicians are key users of health information exchanges (HIE). Understanding their perspectives on the usability of HIE is important if the full potential of the HIE is to be achieved. The literature identified that emergency physician experiences with HIEs are unexplored areas requiring further studies. The purpose of this study using grounded theory methods was to understand the perspectives of emergency physicians concerning the usability of HIEs. The fundamental question was how do emergency physicians use the HIE in making clinical decisions?

Rich and thick data were collected from 15 emergency physicians in four urban hospitals in the mid-south using theoretical sampling and unstructured face-to face interviews. Concepts from the coded segments were developed into categories and an overarching theoretical scheme visualized in a conceptual framework. A substantive theory emerged that using the HIE among emergency physicians is the process of rationalizing non-use and reconciling challenges and benefits. The antecedent of usability was a typical day in the emergency department and why participants accessed the HIE and under what conditions. Six major themes emerged: using the HIE, influencing clinical decisions, struggling with challenges and barriers, recognizing benefits, current views, and rationalizing not using or reduced use of the HIE.

Emergency physicians gave good reasons why the HIE is not being used for the majority of patients while reconciling the challenges and benefits of using the HIE to explain the role of HIEs in making clinical decisions. There was a disconnect in the necessity of using the HIE to make clinical decisions and any negative outcomes that may occur in patients from not using the HIE. Generally, emergency physicians viewed the HIE as not being user-friendly and that they probably do not use the HIE as much as they could for making clinical decisions. The perspective of the emergency physicians was the emergency environment is too busy and because the HIE is less than user-friendly as needed by physicians to practice emergency medicine, the HIE disrupts workflow and is a deterrent to consistent usage in making clinical decisions.

A better understanding of how emergency physicians decided to use the HIE in making clinical decisions gives insights about how to achieve HIE usability. Satisfied end-users who view the HIE as effective and efficient should use the HIE more. However, this requires removing challenges and barriers while recognizing more benefits to using the $\mathrm{HIE}$, and addressing the underlying reasons for not using the HIE. Understanding the complexities of using the HIE and providing solutions to increase usability of the HIE is necessary to influence greater use of the HIE in clinical decisions with demonstrated positive outcomes for patients.
\end{abstract}

\section{Document Type}

Dissertation

Degree Name

Doctor of Philosophy (PhD)

Program

Nursing

Research Advisor

Michael A. Carter, DNSc, DNP 


\section{Keywords}

Health Information Exchange, Emergency Medicine, Healthcare Informatics, Electronic Health Record, Health Information Organization, Emergency Departments

\section{Subject Categories}

Emergency Medicine | Health and Medical Administration | Medicine and Health Sciences

\section{Comments}

One year embargo expired May 2012 


\title{
EMERGENCY PHYSICIANS' PERSPECTIVES ON THE USABILITY OF HEALTH INFORMATION EXCHANGE
}

\author{
A Dissertation \\ Presented for \\ The Graduate Studies Council \\ The University of Tennessee \\ Health Science Center
}

\begin{abstract}
In Partial Fulfillment
Of the Requirements for the Degree

Doctor of Philosophy

From The University of Tennessee
\end{abstract}

By

Shirley A. Thorn

May 2011 
Copyright (C) 2011 by Shirley A. Thorn. All rights reserved. 


\section{ACKNOWLEDGEMENTS}

I would like to acknowledge and extend my sincere gratitude to my dissertation chair, Dr. Michael Carter, for his support, guidance, and expertise. I would also like to thank my dissertation committee members, Dr. James E. Bailey, Dr. Patricia A. Cowan, Dr. Diane Todd Pace, and Dr. Teeradache Viangteeravat for their academic guidance, contributions, support, and time. 


\begin{abstract}
Emergency physicians are key users of health information exchanges (HIE). Understanding their perspectives on the usability of HIE is important if the full potential of the HIE is to be achieved. The literature identified that emergency physician experiences with HIEs are unexplored areas requiring further studies. The purpose of this study using grounded theory methods was to understand the perspectives of emergency physicians concerning the usability of HIEs. The fundamental question was how do emergency physicians use the HIE in making clinical decisions?
\end{abstract}

Rich and thick data were collected from 15 emergency physicians in four urban hospitals in the mid-south using theoretical sampling and unstructured face-to face interviews. Concepts from the coded segments were developed into categories and an overarching theoretical scheme visualized in a conceptual framework. A substantive theory emerged that using the HIE among emergency physicians is the process of rationalizing non-use and reconciling challenges and benefits. The antecedent of usability was a typical day in the emergency department and why participants accessed the HIE and under what conditions. Six major themes emerged: using the HIE, influencing clinical decisions, struggling with challenges and barriers, recognizing benefits, current views, and rationalizing not using or reduced-use of the HIE.

Emergency physicians gave good reasons why the HIE is not being used for the majority of patients while reconciling the challenges and benefits of using the HIE to explain the role of HIEs in making clinical decisions. There was a disconnect in the necessity of using the HIE to make clinical decisions and any negative outcomes that may occur in patients from not using the HIE. Generally, emergency physicians viewed the HIE as not being user-friendly and that they probably do not use the HIE as much as they could for making clinical decisions. The perspective of the emergency physicians was the emergency environment is too busy and because the HIE is less than user-friendly as needed by physicians to practice emergency medicine, the HIE disrupts workflow and is a deterrent to consistent usage in making clinical decisions.

A better understanding of how emergency physicians decided to use the HIE in making clinical decisions gives insights about how to achieve HIE usability. Satisfied end-users who view the HIE as effective and efficient should use the HIE more.

However, this requires removing challenges and barriers while recognizing more benefits to using the HIE, and addressing the underlying reasons for not using the HIE. Understanding the complexities of using the HIE and providing solutions to increase usability of the HIE is necessary to influence greater use of the HIE in clinical decisions with demonstrated positive outcomes for patients. 


\section{TABLE OF CONTENTS}

CHAPTER 1. INTRODUCTION ............................................................................................1

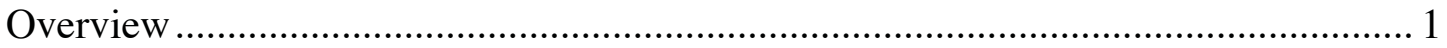

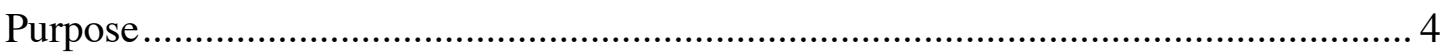

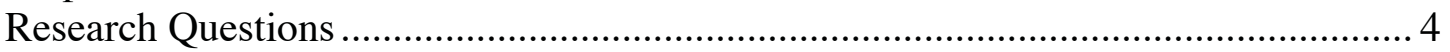

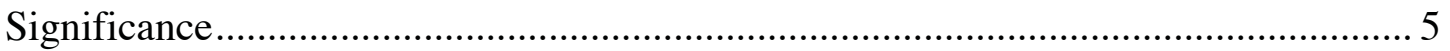

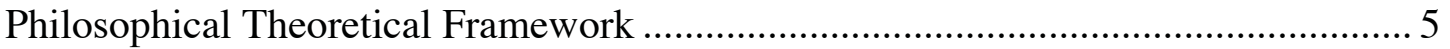

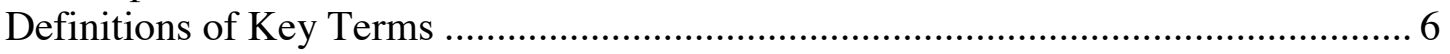

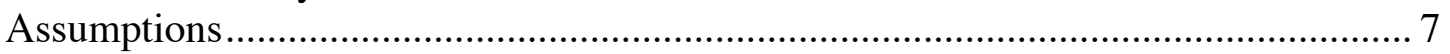

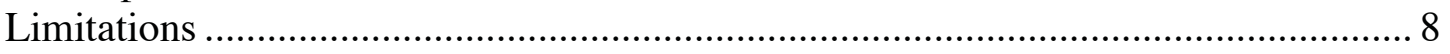

CHAPTER 2. LITERATURE REVIEW ...........................................................10

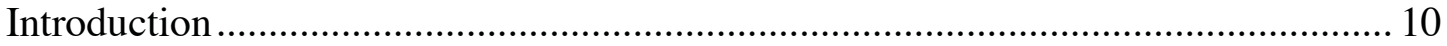

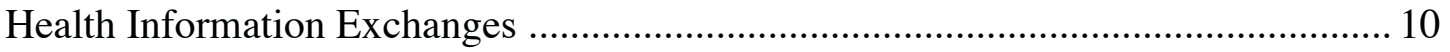

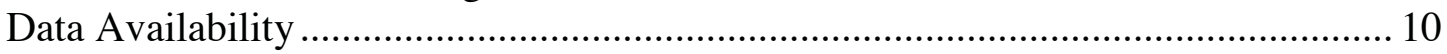

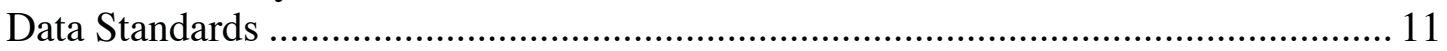

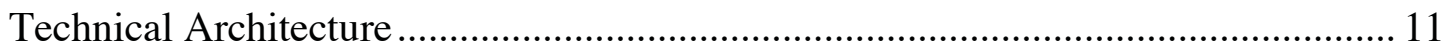

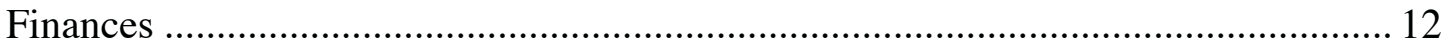

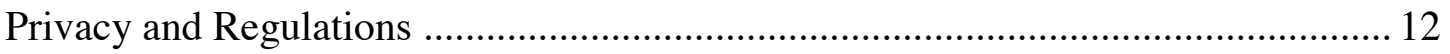

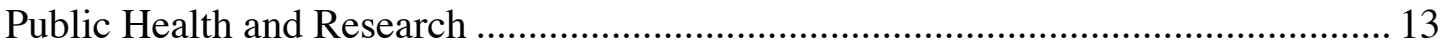

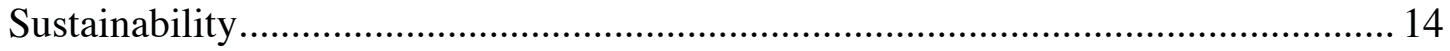

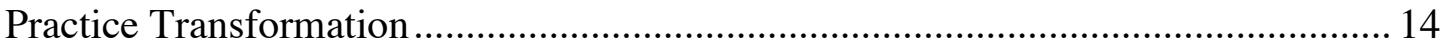

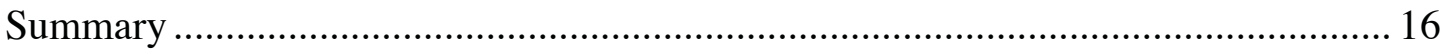

CHAPTER 3. METHODOLOGY .............................................................................17

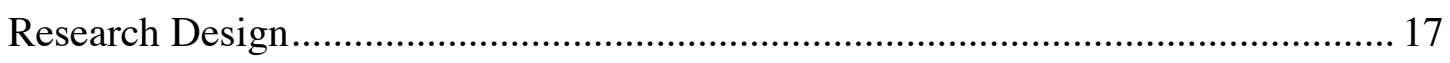

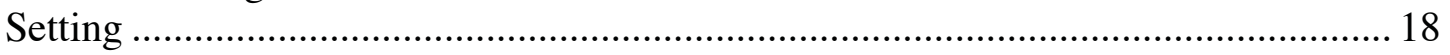

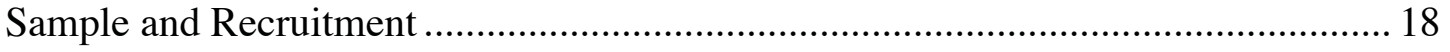

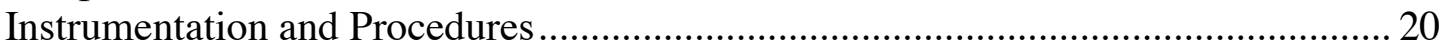

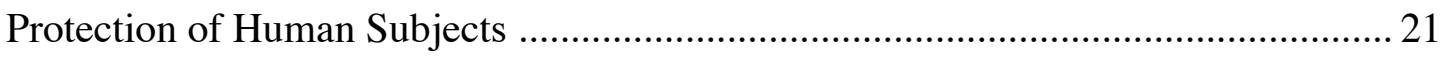

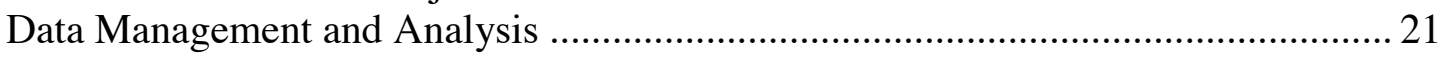

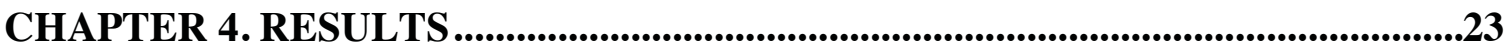

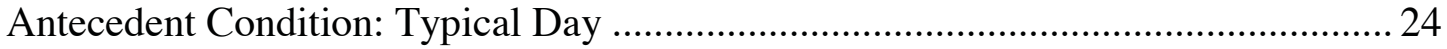

Accessing the HIE during a Typical Day .............................................................. 25

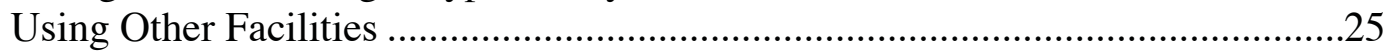

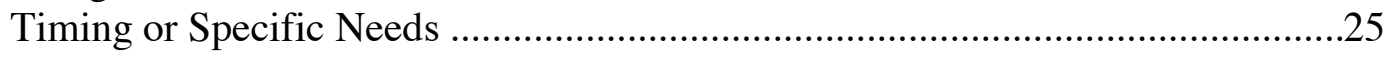

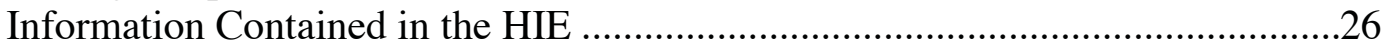

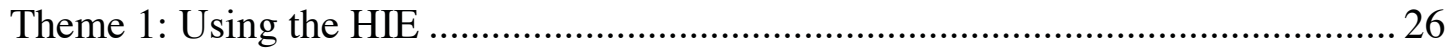

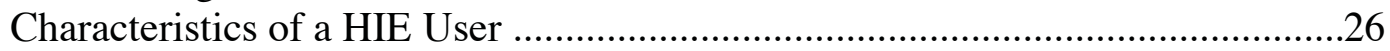

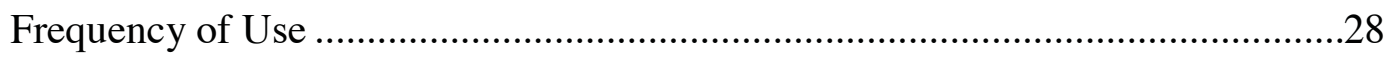

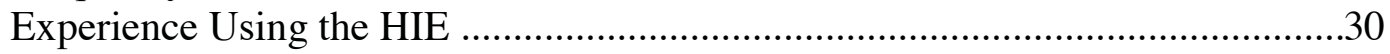

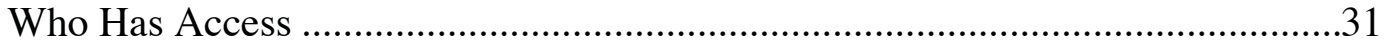

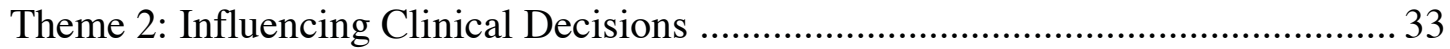

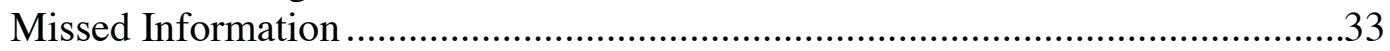




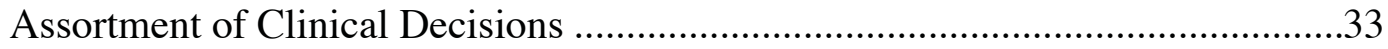

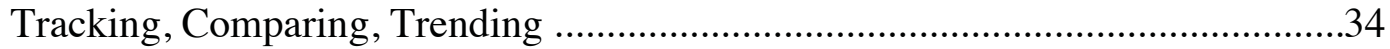

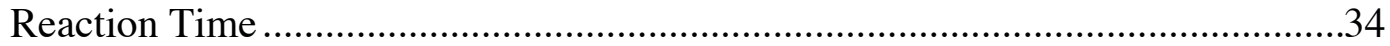

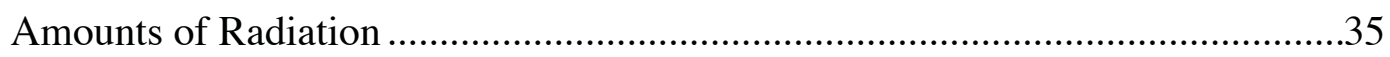

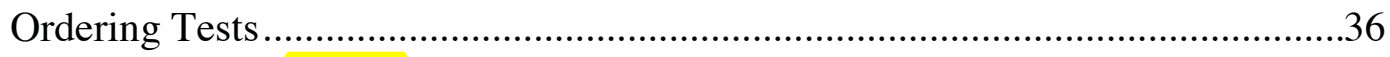

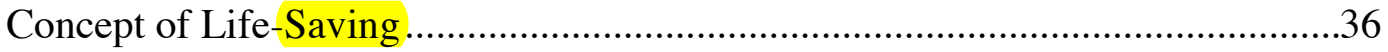

Theme 3: Struggling with Challenges and Barriers ...................................................... 37

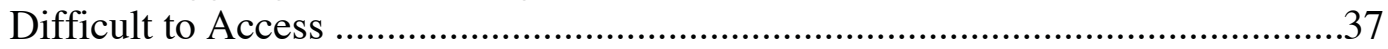

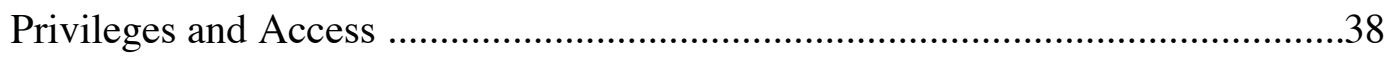

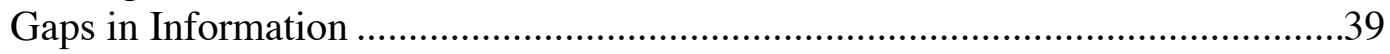

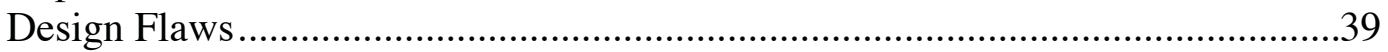

Physician Communication .........................................................................40

Physician input and feedback.................................................................... 40

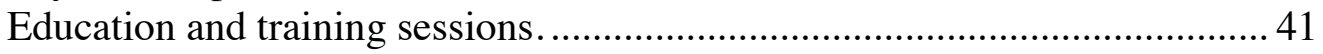

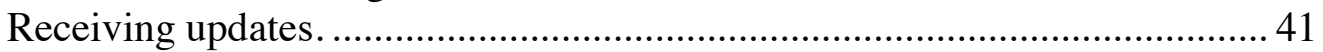

Contact with HIE personnel........................................................................ 41

Communication between emergency physicians............................................ 42

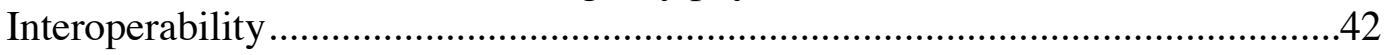

System and software communication. ........................................................... 43

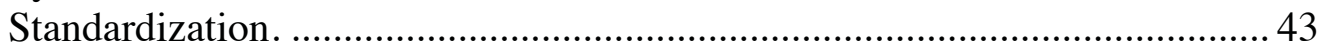

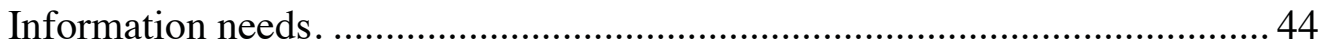

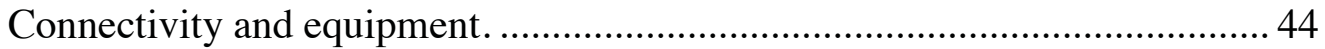

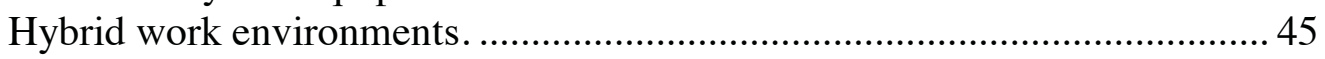

Theme 4: Recognizing the Benefits and Success Factors ........................................... 46

Avoiding Duplicative Testing and Unnecessary Ordering ...................................46

Efficiency in Patient Flow ..............................................................................47

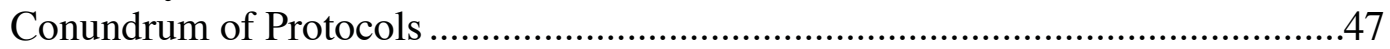

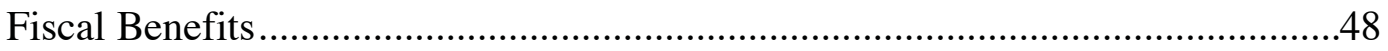

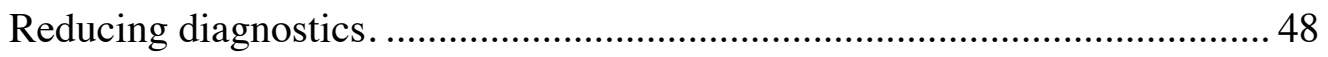

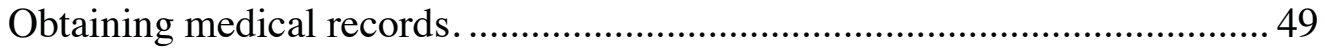

Reviewing records. .................................................................................. 49

Lost productivity with EHRs and HIEs...................................................... 49

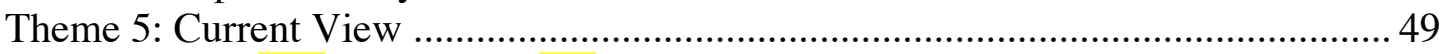

Theme 6: Non-Use or Reduced-use of the HIE ....................................................... 51

Failing to Provide a User-Friendly HIE Coupled with Time Constraints .............53

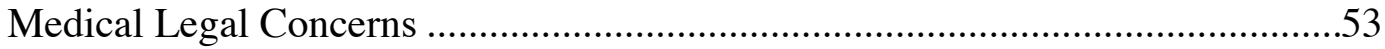

Biasing Clinical Decisions ...............................................................................54

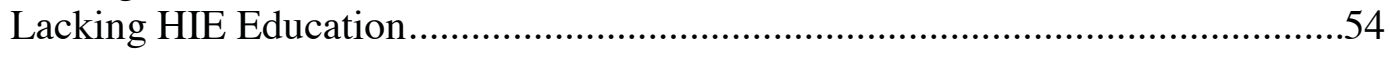

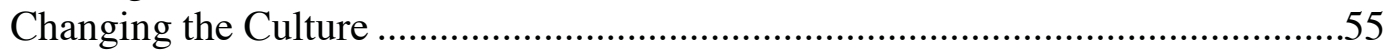

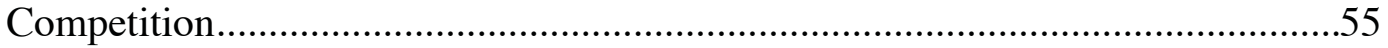

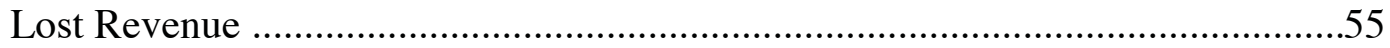

Lacking HIE Champions ...............................................................................56

Failure to Grant Access Privileges........................................................................56

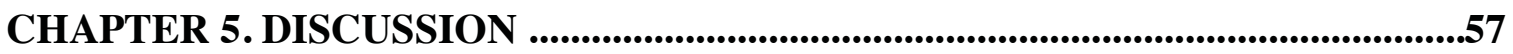

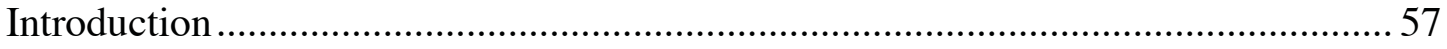

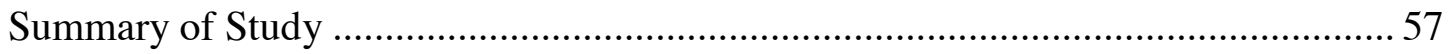




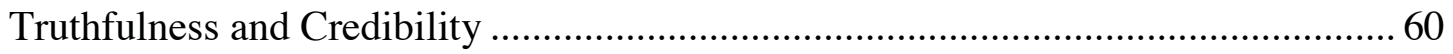

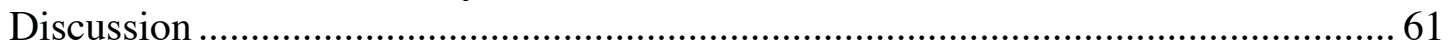

An Analytic Explanation: HIE Usability as Experienced by Emergency

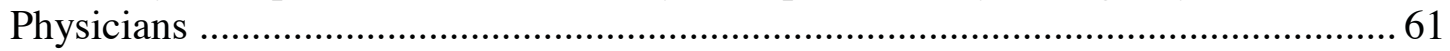

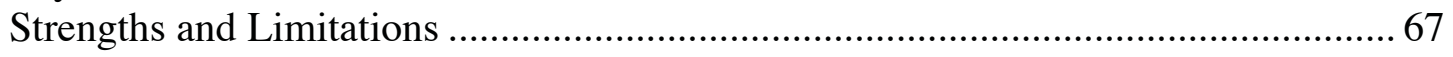

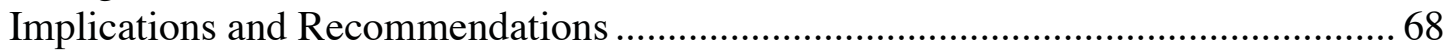

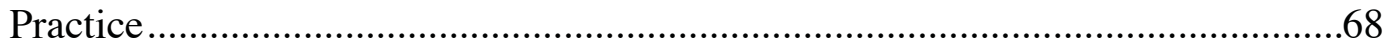

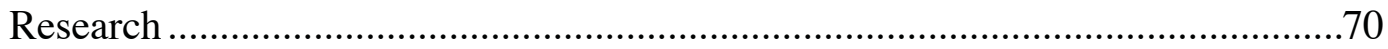

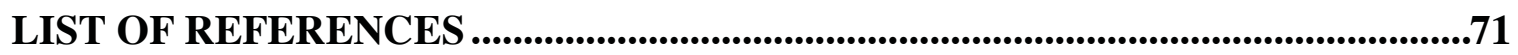

APPENDIX A. LETTER OF INTRODUCTION ........................................................76

APPENDIX B. INSTITUTIONAL REVIEW BOARD APPROVAL ............................79

APPENDIX C. CODING SYSTEM [1132] ...........................................................................80

APPENDIX D. ADDITIONAL EXEMPLARS .................................................................83

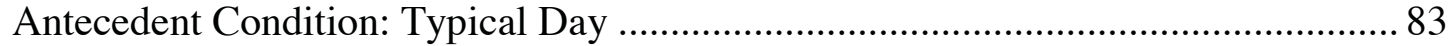

Accessing the HIE during a Typical Day ............................................................... 84

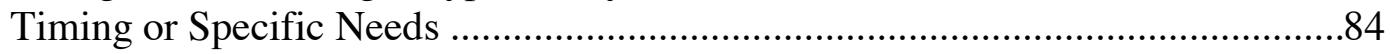

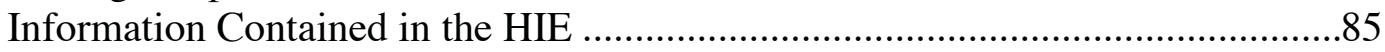

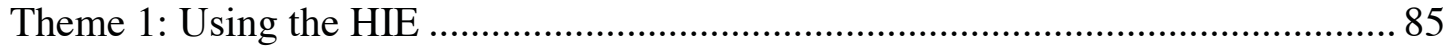

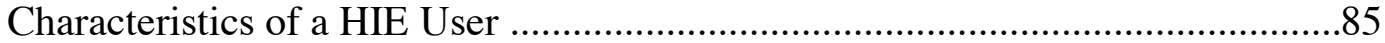

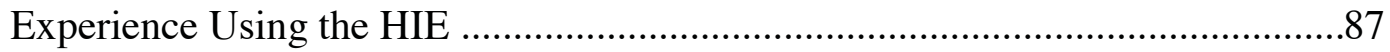

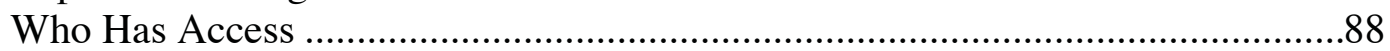

Theme 2: Influencing Care Decisions......................................................................... 88

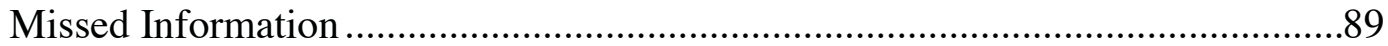

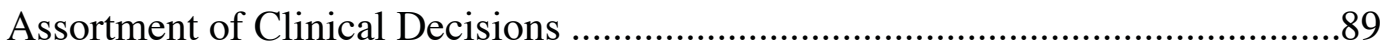

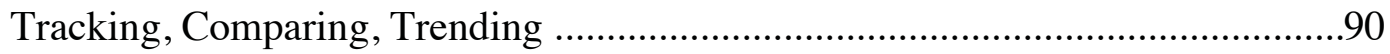

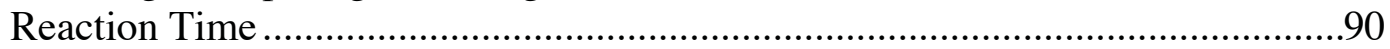

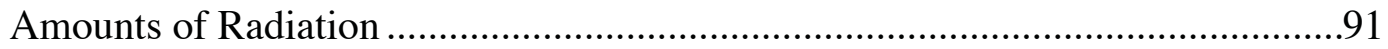

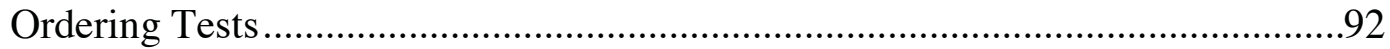

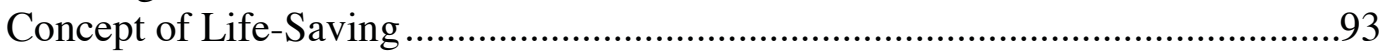

Theme 3: Struggling with Challenges and Barriers ................................................... 93

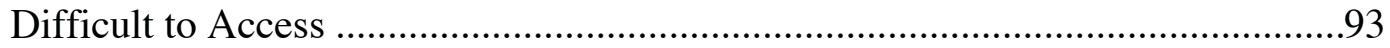

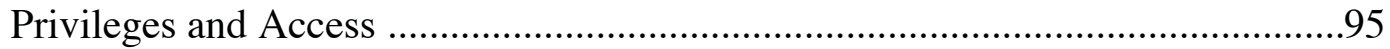

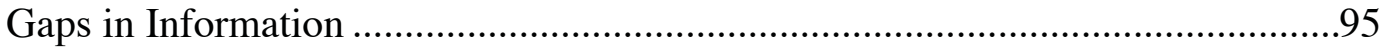

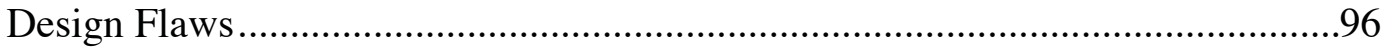

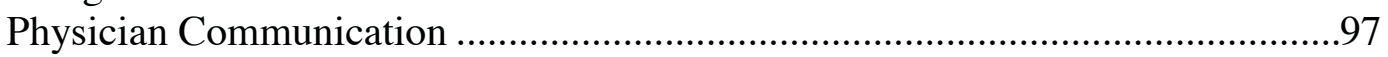

Physician input and feedback ...................................................................... 97

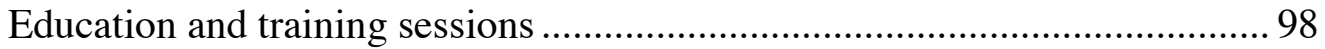

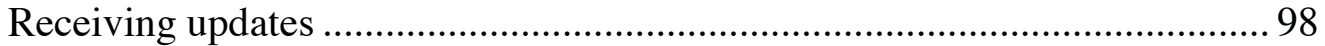

Contact with HIE personnel....................................................................... 99

Communication between emergency physicians …………………….......... 100

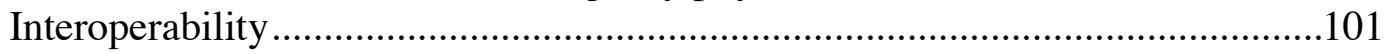

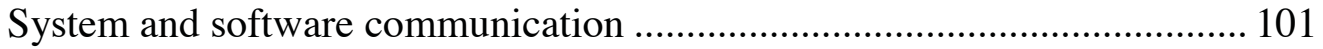




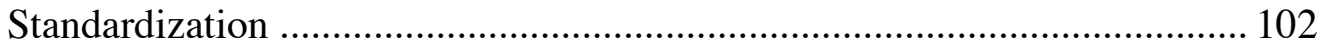

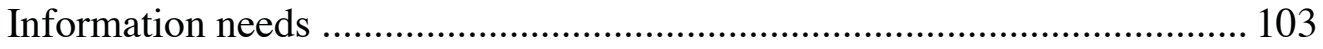

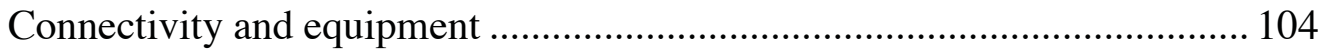

Hybrid work environments .................................................................. 105

Theme 4: Recognizing the Benefits and Success Factors ...................................... 106

Avoiding Duplicative Testing and Unnecessary Ordering ...............................106

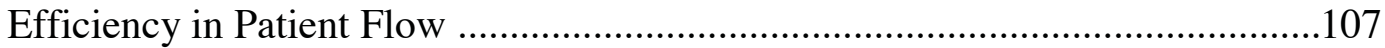

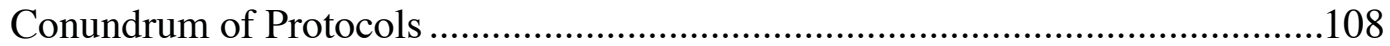

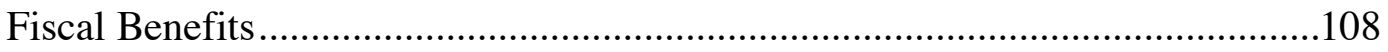

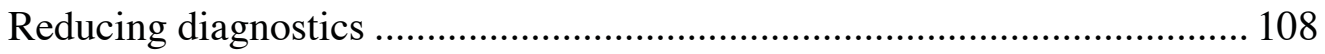

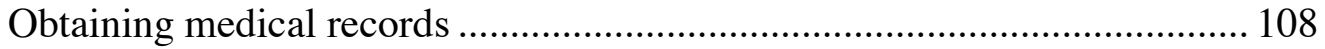

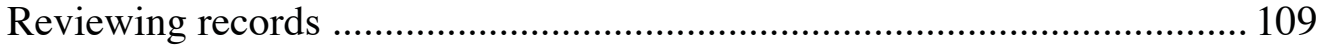

Lost productivity with EHRs and HIEs ............................................... 109

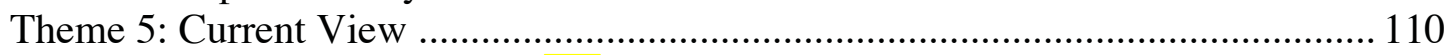

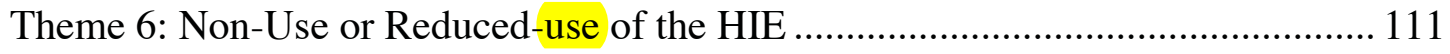

Failing to Provide a User-Friendly HIE Coupled with Time Constraints ...........111

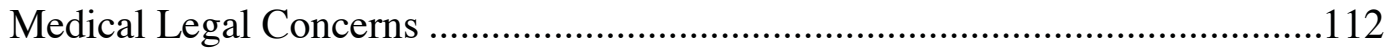

Biasing Clinical Decisions ......................................................................... 113

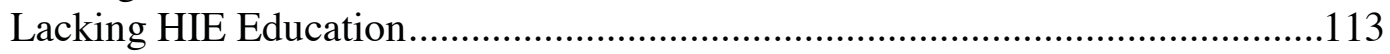

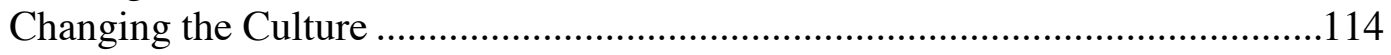

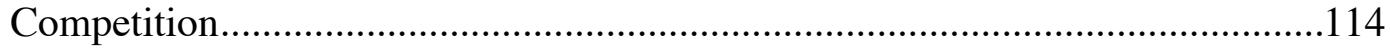

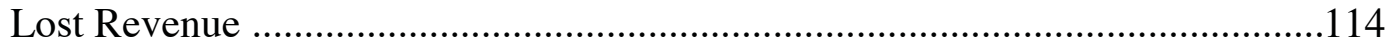

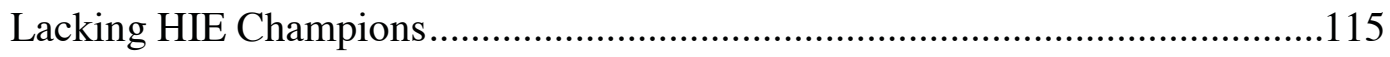

Failure to Grant Access Privileges .............................................................115

VITA 


\section{LIST OF ABBREVIATIONS}

$\begin{array}{ll}\text { AHRQ } & \text { Agency for Healthcare Research and Quality } \\ \text { ARRA } & \text { American Recovery and Reinvestment Act } \\ \text { CT scans } & \text { X-ray computed tomography (Medical Imaging) } \\ \text { ED } & \text { Emergency Department } \\ \text { EGD } & \text { Esophagogastroduodenoscopy } \\ \text { EHR } & \text { Electronic Health Record } \\ \text { EKG } & \text { Electrocardiogram } \\ \text { EMR } & \text { Electronic Medical Record } \\ \text { EMS } & \text { Emergency Medical Service } \\ \text { ER } & \text { Emergency Room } \\ \text { GI } & \text { Gastrointestinal Specialist } \\ \text { HIE } & \text { Health Information Exchange } \\ \text { HIMSS } & \text { Healthcare Information and Management Systems Society } \\ \text { HIO } & \text { Health Information Organization } \\ \text { H\&Ps } & \text { History and Physicals } \\ \text { IOM } & \text { Institute of Medicine } \\ \text { IT } & \text { Information Technology } \\ \text { NHIN } & \text { Nationwide Health Information Network } \\ \text { PHR } & \text { Personal Health Record } \\ \text { RHIO } & \text { Regional Health Information Organization }\end{array}$




\section{CHAPTER 1. INTRODUCTION}

\section{Overview}

"Emergency departments (EDs) in the United States are in crisis" (Shapiro, Kannry, Kushniruk, \& Kuperman, 2007, p. 700). The number of ED visits increased by $26 \%$ to 113.9 million annual visits over a 10 year span of 1993 to 2003 while the number of hospital EDs declined by 12.3\% (425) and hospital beds decreased by 198,000 during the same time period resulting in serious overcrowding (Institute of Medicine, 2006). A nationwide health information network (NHIN) providing health information exchange (HIE) brings patient health data to the point of care, which according to an Institute of Medicine (IOM) Committee report can potentially decrease ED overcrowding and improve efficiency, safety, and quality of health care (Shapiro et al., 2007). In primary care, $15.6 \%$ of all reported errors involve missing clinical information with clinicians viewing the majority of errors as harmful; in addition, a study of emergency physicians found that $15.3 \%$ of patient emergency visits had information missing that likely resulted in patient harm (Smith et al., 2005). An estimated 770,000 adverse drug events (ADE) occur annually with $18 \%$ of the errors attributed to a lack of information at the time of medication decisions (Kaelber \& Bates, 2007).

Kaelber and Bates (2007) reported that HIEs have the potential to influence patient safety by increasing information at the point of care or near the patient, which improves:

1. Drug-allergy information (10\% of ADEs involve significant allergic reactions).

2. Drug-dose information (60\% of prescribing errors are dosing errors).

3. Drug-drug information (medication histories enhance synergistic prescribing, and prevent drug interactions and therapeutic duplications).

4. Drug-diagnosis information (a comprehensive patient diagnosis available at the point of care prevents contraindications between drugs and various clinical states).

5. Laboratory information (ensures indicated lab tests are ordered and results tracked).

6. Radiology information (up to $2 \%$ of abnormal mammograms are not followedup).

7. Communication between providers (the Joint Commission on Accreditation of Healthcare Organizations, JCAHO, identified a major cause of breaks in patient safety is the failure to transfer patient information).

8. Communication between patients and providers (personal health records can improve communication, but integration of personal records requires improved HIEs).

9. Public health information, which promotes surveillance of infectious diseases, biohazards, environmental exposures, and drugs post marketing (Kaelber \& Bates, 2007). 
The 1999 Institute of Medicine report, To Err is Human, identified fragmented health systems as a major cause of errors because providers cannot access patient data (Shapiro et al., 2006). Health care providers need the ability to access timely patient data when the data are needed (Shapiro et al., 2006) since patient safety is dependent on the right information available to the right person at the right time (Kaelber \& Bates, 2007). Because patients use the ED for urgent and unplanned visits, the right information at the time of the patient visit is not always available and emergency physicians order treatment without understanding the patient's complete medical history (Hripcsak, Sengupta, Wilcox, \& Green, 2007). Many of the problems occurring at the point of care result from missing information because patients move between different providers and organizations (Shapiro et al., 2007). Insufficient baseline information forces emergency physicians to make decisions without adequate access to necessary patient data (Shapiro et al., 2007).

A fragmented system stores patient records in multiple locations with some portions of the record electronic and other parts on paper; in addition, information is not shared across organizations or between providers (Grossman, Bodenheimer, \& McKenzie, 2006). Added to this is a fragmented emergency care system caused by a lack of coordination between emergency medical service (EMS) agencies, mismanaged regional patient flow, and divided accountability for the system, which results in a system where it is difficult to determine where failures occur or why (Institute of Medicine, 2006). The existing fragmented health care system could be integrated by sharing electronic health information across settings through HIEs, thereby decreasing costs, utilization, and medication errors while increasing clinical guideline adherence and enhancing surveillance and monitoring (Kern \& Kaushal, 2007; Kern, Barron, Abramson, Patel, \& Kaushal, 2009).

Because of the volume of patients visiting EDs, there are opportunities to link providers and communities through HIEs and influence medical care; however, effectively communicating information to the point of care requires intensive effort, such as:

1. Identifying patients accurately.

2. Accessing correct patient data across healthcare systems.

3. Authenticating provider identification.

4. Standardizing patient records.

5. Ensuring confidentiality and privacy of patient data (Finnell, Overhage, \& McDonald, 2005).

More studies are needed to identify the data needs of emergency physicians combined with the best methods to implement systems so that HIEs are integrated with clinical workflow (Shapiro et al., 2007). Qualitative studies would be beneficial in the evaluation of HIE systems given the unanticipated effects on clinical workflow (Johnson $\&$ Gadd, 2007). HIE adoption by emergency physicians is affected by how the data are presented and how accessible the data are at the point of care since physicians need easy and rapid access to information (Shapiro et al., 2007). One study reported that emergency physicians thought patient disposition times would increase with HIEs because the access 
to additional information would require more time to obtain and synthesize (Shapiro et al., 2007). However, different results were reported when expanding beyond the specialty of emergency physicians and studying a population of licensed physicians. A mail survey $(n=1043)$ of practicing physicians in Massachusetts reported that the HIE would have a positive effect by reducing costs (70\%), improving quality (86\%), and saving time (76\%); however, they also indicated concerns about privacy (71\%) and were reluctant to pay for participating (Wright et al., 2010).

Reducing ED costs requires physicians to alter workflows by accessing and considering HIE information prior to initiating treatment plans (Frisse \& Holmes, 2007). The Mount Sinai Medical Center ED in New York City improved patient flow by redesigning workflows and implementing a fully integrated ED information system with direct access to historical patient data in a hospital repository (Baumlin et al., 2010). The Indiana Network for Patient Care compiled information over a four year period from 19 facilities and 12 EDs that demonstrated a substantial portion of healthcare is delivered through emergency visits (50\% of the Indianapolis population) and therefore, the hospital information infrastructure is an important component of the HIE (Finnell et al., 2005).

HIEs have the potential to impact ED workflow efficiency by decreasing redundant testing and throughput times, reducing unnecessary admissions and the number of admitted patients waiting for beds, and decreasing the number of patients waiting to be seen or leaving the ED without being seen (Shapiro et al., 2006). However, a database study of 4,830 U.S. hospitals reported that adoption and implementation of HIEs are different phenomena with many non-technological factors a barrier to an effective HIE, such as increased ED volume or competition between healthcare organizations (Vest, 2010). A 2007 survey of 145 regional health information organizations (RHIOs), which organizes all the components of a HIE into an underlying local infrastructure, found that nearly one in four is non-functioning, which questions the viability and sustainability of widespread HIE implementation (Adler-Milstein, McAfee, Bates, \& Jha, 2008). The IOM (Institute of Medicine, 2006) stated that in order to determine how to deliver emergency services more research is needed. Few studies have explored how understanding emergency physician views and experiences with HIE capabilities might change how HIEs are used in making clinical decisions or how often HIEs are accessed when making decisions. In addition, understanding the needs of emergency physicians from their perspective may encourage more effective HIE usage that will contribute to long-term HIE sustainability.

The NHIN is receiving public policy attention because of the importance in improving efficiency and safety of health care in the United States, but adoption has been limited (Kaushal et al., 2005). The American Recovery and Reinvestment Act (ARRA) of 2009 committed more than 19 billion dollars to expand health information technology along with electronic documentation and fulfilling the President's goal of every American having access to an electronic health record (EHR) by 2014 (Blumenthal, 2009). The Beacon Community Cooperative Agreement Program, which is within the ARRA, supports communities with high information technology (IT) adoption rates by funding demonstration projects that improve quality, cost efficiency, and population 
health (Maxson et al., 2010). The federal money, which is focused on health information technology expansion, is expected to expand EHRs as organizations and physicians implement EHRs to qualify for incentive payments (Blumenthal, 2009; Wright et al., 2010). However, only $17 \%$ of physicians and $10 \%$ of hospitals currently have basic EHRs, which is only one of the challenges facing HIEs (Blumenthal, 2009).

In addition to the Beacon Communities Program, the federal government is supporting state-level HIEs (Conn, 2011), but HIEs are immature and there is more to understand with little research conducted in this area (Ash \& Guappone, 2007). Qualitative methods are useful for formative evaluations so that information can be applied to HIE development (Ash \& Guappone, 2007). Qualitative studies are needed to understand the environment, HIE capabilities, needs assessments, and usage (Johnson \& Gadd, 2007).

\section{Purpose}

The purpose of this qualitative study was to understand the perspectives of emergency physicians concerning the usability of HIEs. The relevance of this study is the potential to increase the understanding of what emergency physicians go through and have to do if they want to use the HIE system. This study also has implications for how healthcare is delivered to patients visiting hospital emergency departments. The aim of this study was to elicit emergency physicians' perspectives that would explore core concepts of how emergency physicians use the HIE system and to provide a theoretical explanation that will provide helpful information in the development of appropriate interventions that facilitate emergency physician use of the HIE.

\section{Research Questions}

A fundamental research question was how do emergency physicians use the HIE in making clinical decisions? During the course of the research, the following specific questions emerged from the collected data and analysis:

1. What did participants view as essential information for emergency physicians in the HIE?

2. What did participants view as characteristics of being a user or non-user of the HIE?

3. How did participants view they were using the HIE in making clinical decisions?

4. What factors do participants view as being challenges or barriers to accessing and using the HIE?

5. What elements did participants view as necessary for the HIE to be successful?

6. How did participants currently view the HIE? 


\section{Significance}

Data are necessary to answer the questions of how emergency physicians are using health information exchange systems, or how data repositories can lead to improved outcomes and long term health care savings. Qualitative studies are needed to better understand what physicians view as important factors in HIE adoption (Rudin, Simon, Volk, Tripathi, \& Bates, 2009). Understanding the needs and views of emergency physicians is important to increase HIE usage and demonstrate improved care decisions, decreased duplicate services, reduced costs, and decreased patient time spent in the ED. The literature suggests that emergency physician views and experiences with HIEs are largely unexplored areas and further studies are needed.

This study contributes to the literature by learning what emergency physicians want in a HIE system, from the point of view of the emergency physicians and thus, understanding why emergency physicians are adopting or not adopting the capabilities of HIEs. The primary significance is to gain insight of how emergency physicians use the HIE to make clinical decisions. Understanding the needs of emergency physicians and what it takes for them to access the HIE is necessary in order for emergency physicians to use the HIE. Knowing what works and what does not work in relation to HIEs is challenging because knowledge of needs, expectations, and motivations of physicians is lacking (Ash as cited in Rudin et al., 2009).

\section{Philosophical Theoretical Framework}

A conceptual framework was not located during the literature review likely because there are few studies published that examine the usability of HIE capabilities from the perspective of emergency physicians. A framework results from the outcome of qualitative studies (Burns \& Grove, 2009) and there are few qualitative studies exploring emergency physician perspectives. Corbin and Strauss (2008) do not begin studies with a set of concepts, although they indicated that a theoretical orientation is necessary in order for the researcher to develop a research approach and justify the study methodology.

This study was guided by Corbin and Strauss (2008) who use an interactionist and pragmatist epistemology approach to research. The interpretivist perspective focuses on understanding how individual emergency physicians report their experience and view of HIEs, and what access to the health information exchange system means for them. There are multiple versions of reality, or the meaning of the phenomena of HIEs as viewed by emergency physicians, because they are making sense of their own experiences. This researcher engaged in data generation and analysis while co-producing knowledge through reflexivity by using a postmodern epistemology approach of socially constructed reality for identified concepts during data analysis. This was an iterative and ongoing process with the interpretations of the text obtained from interviews of the phenomena while incorporating the researcher's knowledge (Corbin \& Strauss, 2008). Using the guidance of grounded theory techniques, the fit between the initial research topic and the emerging data was evaluated by exploring the views and experiences of emergency 
physicians using HIEs and discarding concepts that proved to be irrelevant. Grounded theory techniques increased the flexibility to add pieces and follow leads, even late in the analysis, which increased the focus and provided clearer detail of what was happening in the data (Corbin \& Strauss, 2008).

Constructivist grounded theory, which is part of the interpretive tradition, prioritized the phenomena of the study while recognizing that both the data and analysis are the result of both researcher and participants interpreting the meanings and actions (Charmaz, 2006). Using a symbolic interactionism theoretical perspective assumed that interaction is interpretive and recognized that there is a relationship between meaning and actions (Charmaz, 2006). Using a grounded theory approach for data generation, unstructured interviews allowed emergency physicians to share their experiences, perspectives, and understandings surrounding HIEs. The aim of the interviews was to give individual emergency physicians the opportunity to discuss and present their experiences with HIEs. There was a need to understand how emergency physicians were using HIEs: (a) How did emergency physicians view HIEs? (b) How did emergency physicians engage with HIEs? and, (c) What did emergency physicians report as the effect of using or not using HIEs on their clinical decisions? Considering the study as a whole, a grounded theory approach of not forcing the data guided the researcher through the data collection and analysis to learn about the views and experiences of emergency physicians, and to understand the phenomena from their perspective.

\section{Definitions of Key Terms}

The following definitions guided various components of this research and were used in this study:

Health Information Exchange (HIE): "Electronic movement of health-related information among organizations according to nationally recognized standards" (The National Alliance for Health Information Report, 2008, p. 22).

Regional Health Information Organization (RHIO): “A health information organization that brings together health care stakeholders within a defined geographic area and governs health information exchange among them for the purpose of improving health and care in that community" (The National Alliance for Health Information Report, 2008, p. 22).

Health Information Organization (HIO): "An organization that oversees and governs the exchange of health-related information among organizations according to nationally recognized standards" (The National Alliance for Health Information Report, 2008; The National Alliance for Health Information Report, 2008, p. 22).

Electronic Medical Record (EMR): "An electronic record of health-related information on an individual that can be created, gathered, managed, and consulted by authorized 
clinicians and staff within one health care organization" (The National Alliance for Health Information Report, 2008, p. 15).

Electronic Health Record (EHR): "An electronic record of health-related information on an individual that conforms to nationally recognized interoperability standards and that can be created, managed, and consulted by authorized clinicians and staff across more than one health care organization" (The National Alliance for Health Information Report, 2008, p. 15).

Personal Health Record (PHR): "An electronic record of health-related information on an individual that conforms to nationally recognized interoperability standards and that can be drawn from multiple sources while being managed, shared, and controlled by the individual" (The National Alliance for Health Information Report, 2008, p. 15).

Usability: "...the effectiveness, efficiency, and satisfaction with which specific users can achieve a specific set of tasks in a particular environment" (HIMSS EHR Usability Task Force, 2009, p. 3).

Nationwide Health Information Network (NHIN): “A national effort to establish a network to improve the quality and safety of care, reduce errors, increase the speed and accuracy of treatment, improve efficiency, and reduce health care costs" (TN Office of eHealth Initiatives, 2009, p. 46).

\section{Assumptions}

This study involved observing and interacting with emergency physicians in a busy environment and a major assumption was that the physicians would be amendable to face-to-face interviews regarding their use and views of the HIE system. Discussing how physicians make care decisions and the role of HIE systems in the process might be conceived by some participants to be sensitive information. This researcher assumed that being a registered nurse with extensive clinical and management experience in emergency departments would be beneficial when interacting with health care professionals during the study and eliciting sensitive information from participants. Understanding the jargon of electronic documentation and emergency workflow was essential. Qualitative research uses a technique known as bracketing to suspend what the researcher knows about the phenomena being studied (Burns \& Grove, 2009). However, Charmaz (2006) maintains that intimate familiarity with the phenomena allows the researcher not to take for granted what the participants assume but to question their own perspectives. Because the researcher shared a common culture with the research participants, the researcher was able to draw on experiences to gain insights into the participants' descriptions and explore other possible meanings (Corbin \& Strauss, 2008). Relating to your own experiences allows the researcher to use those properties and dimensions and to look more closely at the data (Corbin \& Strauss, 2008). 
The researcher examined the hidden assumptions in the participants' language during coding and interpreted their implied meanings, such as the computer cannot do a particular function, when in fact the participant was not familiar with the functions. The process was interactive as the researcher refined the codes with each successive interview and as the researcher tried to understand each participant's views and actions from their perspective. The researcher questioned everything and sought out cases that did not fit the pattern, such as participants who did not have privileges to access the HIE.

Assumptions for this study included the following:

1. There is no difference in usage rates among emergency physicians based on gender, age, or race. The study sample was heterogeneous for gender, age, and race.

2. Data gathering in the workplace is more convenient for emergency physicians. The interviews were scheduled in the emergency departments according to times and dates selected by the participants.

3. The usage rates for the HIE system in the study sites has been reported to be less than $20 \%$. The usage rates of the HIE system in the clinical setting as reported by the participants might differ from the rates the researcher had read in reports.

\section{Limitations}

The following were considered limitations in this study:

1. The study sites are in one geographical area, a mid-south city and therefore, the results may not reflect the views of emergency physicians in other geographical areas of the country.

2. The study sites are located in urban hospitals and the results may not be representative of emergency physicians practicing in community hospital settings.

3. HIEs are in various stages of development. This study will reflect the current state of HIE development and implementation in this geographical area, which may not be consistent with other areas of the country.

4. This study is one point in time (cross-sectional design) and emergency physicians may need to be followed over time in subsequent studies to understand the problems and complexity of the issues completely.

5. The key decision makers in the emergency departments of the study sites are emergency physicians; however, this study recognizes that nurse practitioners and physician assistants also use the HIE system, but they do not constitute the majority of the provider population in emergency departments in the study sites. Therefore, this study limits the participants to emergency physicians, but recognizes that further studies are needed to focus on providers other than physicians.

6. This study is limited to adult patient populations. The RHIO for this geographical area has not incorporated pediatric data information into the HIE; therefore, the results may not be applicable to a study population of pediatric hospitals. 
7. Theoretical saturation or sufficiency occurred when the researcher determined that the properties, dimensions, and variations of each category were sufficiently developed for the purposes of this research and may be considered a limitation of this study. 


\section{CHAPTER 2. LITERATURE REVIEW}

\section{Introduction}

The following is a review of literature relevant to health information exchange usability and emergency physicians that helps formulate the research question for this study, enhances the sensitivity of this qualitative study, and stimulated questions during data analysis. In qualitative research, literature reviews provide initial questions, concepts, and ideas for theoretical sampling, but must not hinder the creativity of the study (Charmaz, 2006). Most published literature has not focused on emergency physicians and little is known about emergency physicians' perspectives of health information exchange (Shapiro et al., 2007). This review of literature will address the issues related to health information exchange use and factors identified as important in successful adoption and usability.

\section{Health Information Exchanges}

For the successful adoption of health information exchanges (HIE), the following issues need to be addressed: data availability, data standards, technical architecture, finances, privacy, and public health and research (Shapiro et al., 2006). In addition, Kern et al. (2009) reported that financial sustainability, technical architecture, and practice transformation were necessary for interoperable health information technology. AdlerMilstein, Bates, and Jha (2009) reported that barriers to HIE development included privacy and security concerns, legal/regulatory challenges, and unexpected high costs of operating HIEs. Overall, the successful adoption of HIEs encompasses all these factors.

\section{Data Availability}

There needs to be increased electronic health record (EHR) implementation to capture larger amounts of clinical data and make HIEs successful on a large scale (Shapiro et al., 2006). With more information and more people exchanging health information, patient safety will improve because more data provides a better clinical picture (Kaelber \& Bates, 2007). Missing clinical information has been implicated in $15.6 \%$ of reported errors in primary care; one study reported that important clinical information is missing in one out of every seven visits (Smith et al., 2005). Providers need access to clinical data, but also need to control how and where the results are delivered (McIlwain \& Lassetter, 2009). Providers must be able to access data that are timely, and when and where the data are needed (Shapiro et al., 2006). Many problems are due to information gaps because baseline information is missing, which results in emergency providers making decisions without relevant patient data (Shapiro et al., 2007). Integrating HIEs into clinical workflows is crucial to prevent delayed access to information that further impairs usability (Rudin et al., 2009). However, integrating HIEs 
into workflow is not a one-time process and needs to occur with hardware, software, policies, and content (Johnson \& Gadd, 2007).

Broader interconnecting of providers and information technology (IT) systems with remote access is needed in a fragmented system where patient records are stored in multiple locations (Grossman et al., 2006). The systems have limited ability to communicate with each other even with HIE capabilities, especially with unaffiliated providers (Grossman et al., 2006). However, HIEs do have the potential to integrate an existing fragmented health care system while decreasing costs (Kern et al., 2009).

\section{Data Standards}

In 1999, the Institute of Medicine (IOM) report, To Err is Human, recommended data standards to reduce errors caused by providers unable to access patient data in fragmented systems (Shapiro et al., 2006). The absence of data standards are a major barrier to widespread HIE implementation, largely due to the customization required to match data elements (Shapiro et al., 2006). Healthcare data standards as applied to HIEs include: methods, terminologies, and specifications for information collection, exchange, storage, and retrieval associated with electronic health records (Saba \& McCormick, 2006). Current technology, which transfers large amounts of data, is not proficient in managing the ambiguity in structure and meaningful content of the data (Saba \& McCormick, 2006). Standards reduce ambiguity and actions are based on consistent data with the actual meaning intact (Saba \& McCormick, 2006). Standards are lacking coupled with informatics applications that fail to exchange information effortlessly because of design (Englebardt \& Nelson, 2002). There are a number of technical standards and committees but they lack a single standardizing body; thus, informatics applications do not share information easily (Englebardt \& Nelson, 2002) and the number of standards impairs integration of electronic health record (EHR) capabilities and HIE interoperability. The results are a barrier to HIE implementation.

\section{Technical Architecture}

Technical issues and workflow integration are concerns for providers (Kern et al., 2009). Providers will abandon systems that are not designed with usability in mind for the end-users (Kern \& Kaushal, 2007). For example, regional health information organizations (RHIO) are using various file sharing models, which creates a challenge for matching patient records (Shapiro et al., 2006). Consequences of poor design include increased workloads and disrupted work routines for providers, and an increased potential for errors in care (Kern \& Kaushal, 2007). Usability engineering guides design, evaluation, and implementation of information systems and is studied for predicting interface design problems and improving safety of information systems (Kushniruk, Triola, Borycki, Stein, \& Kannry, 2005). Kushniruck (2005) explored the relationship between system usability and medical error (technology-induced error) and reported a variety of usability problems with the majority of the problems related to interface design 
issues. The study, which is part of a larger research program studying system usability to predict interface design problems, suggested that display visibility and default values have a high probability of errors (Kushniruk et al., 2005). Other solutions included single 'sign-on' to view aggregated patient data, which is more user-friendly for some of the fragmented system issues (Grossman et al., 2006).

\section{Finances}

Preliminary findings by Frisse and Holmes (2007) suggested that urban regions can realize annual savings if HIE data is used at the point of care in emergency departments (EDs) and non-acute visits are shifted to ambulatory care settings. Shapiro et al. (2006) cited studies estimating that access to patient data reduced an ED visit by 26 dollars, while another study estimated a decrease of five dollars, thereby estimating annual savings of between 570 million dollars to 2.9 billion dollars in emergency departments across the United States. Although various studies are projecting cost saving with a nationwide HIE network to be in the billions of dollars per year, the authors also point out that the savings are presumed and further research is necessary to demonstrate the actual realization of benefits in a variety of settings (Shapiro et al., 2006). An expert panel estimated that a model national health information network (NHIN) would require 156 billion dollars in capital investment and 48 billion dollars in annual operating costs to achieve the model in five years (Kaushal et al., 2005).

HIEs have the potential to positively impact ED workflow efficiency by decreasing: redundant testing, throughput times, unnecessary admissions, number of admitted patients waiting for beds, number of patients waiting to be seen, and number of patients who leave the ED without being seen (Shapiro et al., 2006). Frisse and Holmes (2007) concluded that the behavior of emergency physicians is influenced by liability and workflow concerns, availability of information, and the reluctance to use results from other institutions, which causes repeated testing. Further studies are needed to show that available information will lead to behavior changes because actual cost savings are dependent on behavior changes by both patients and providers (Frisse \& Holmes, 2007). It is recognized that changes in behavior are difficult. Involving emergency physicians in designing the HIE systems will ensure their needs as end-users are met (Shapiro et al., 2006). Increasing the adoption of HIEs will help improve efficiency while addressing ED overcrowding and improving safety and quality (Shapiro et al., 2007).

\section{Privacy and Regulations}

Adler-Milstein et al. (2009) identified that HIE adoption is impaired by concerns over privacy and security, and competitiveness in the marketplace over the ability to retain patients with the free flow of clinical data. Rudin et al. (2009) reported that security concerns were a priority for stakeholders and HIE success depends on how well the issue of trust is addressed. Ideally, automated patient information would be available

nation-wide and securely accessed by authorized health care providers (Shapiro et al., 
2006). The Health Insurance Portability and Accountability Act, known as HIPPA regulations, allow for the communication of patient information without explicit patient consent, but there is concern that HIEs could potentially increase inappropriate access to patient information (Shapiro et al., 2006). Unfortunately, privacy policies related to HIEs are slow to come forward (Shapiro et al., 2006). Qualitative studies are needed to understand the environment, HIE capabilities, needs assessments, and usage (Johnson \& Gadd, 2007).

\section{Public Health and Research}

Public health databases of reportable diseases, vaccination registries, and biosurveillance are being created by HIEs with opportunities for clinical research (Shapiro et al., 2006). The secondary use of health data can augment population health outcomes by supporting public health and facilitating earlier detection of epidemics or bioterrorist threats (Hripcsak et al., 2009; Mandl et al., 2004). EHRs provide detailed clinical data that will increase the depth of information available for non-clinical applications, such as increased knowledge of diseases and treatments, quality and safety in health care delivery, pay for performance and reimbursement, and efficiency and effectiveness of health systems (Safran et al., 2007). Aggregated data available with HIEs provide a wider range of public health applications, including evolving public concerns, emergency preparedness, global epidemiology, and homeland security (Safran et al., 2007).

To increase bioterrorism preparedness following 2001, biomedical, public health, defense, and intelligence communities developed approaches to real-time disease surveillance to augment existing systems (Mandl et al., 2004). Syndromic surveillance, which is the detection of individual and population health indicators before a confirmed diagnosis, including before laboratory confirmation, quickly evolved (Mandl et al., 2004). Syndromic surveillance technology is particularly useful for public health organizations in the identification of clusters of undiagnosed disease, recurrent cyclic disease, dynamics of hospital visits, trending, and the presence or absence of a particular disease epidemic (Mandl et al., 2004). Public health authorities have an opportunity to perform many applications pertinent to population health outcomes with the increased adoption of EHRs, such as comprehensive surveillance of comorbidities, duration of symptoms, and travel history (Hripcsak et al., 2009). In addition, linking syndromic surveillance programs to public health can monitor problems and regional patterns, such as asthma and chronic health trending (Mandl et al., 2004). The recent influenza epidemics in 2010 raised awareness of a potential pandemic and the importance of syndromic surveillance technology, or lack of, received renewed attention.

The nation is experimenting with using EHRs for syndromic surveillance based on ED chief complaints; however, the use of ED data requires engineers to customize surveillance systems to the data source because of the lack of health care standards (Hripcsak et al., 2009). Additional technology problems, such as institutions developing 
their own terminologies and the lack of standards for data entry, have profound effects on syndromic surveillance performance (Hripcsak et al., 2009).

\section{Sustainability}

Rudin et al. (2009) found that sustainability of HIEs is a concern when organizations can no longer rely on grants. There are few examples of sustainable HIEs to serve as models. A 2007 survey found that out of more than 100 HIE organizations, one quarter were defunct (Rudin et al., 2009), while another survey in the same year found a $26 \%$ failure rate (Adler-Milstein, Bates, \& Jha, 2009). A barrier to the development of HIEs is the lack of funding, while the country's market-based approach is a concern considering stakeholders are needed to fund RHIOs (Adler-Milstein et al., 2009). Competition is seen as a barrier to the adoption of HIEs when providers must collaborate and share patient data, which is their most valued asset (Grossman et al., 2006). It is essential to understand if RHIOs are successfully exchanging data and if the current approach to a nationwide HIE will be successful (Adler-Milstein et al., 2009). RHIO viability is worrisome considering the high failure rates with limited HIE saturation (Adler-Milstein et al., 2009); thus, increasing HIE usage and demonstrating cost savings is imperative for future sustainability. Since we have a free market system, which results in competition and inconsistencies in HIE systems, a nationwide system would provide consistency and make the HIE more usable.

\section{Practice Transformation}

Evolving HIE systems provide added features that meet the needs of the providers and promotes information technology adoption, especially customized features that are commonplace in the business world to expedite and secure transactions (Shapiro et al., 2006). Understanding the needs of providers is important to ensure their needs are being met (Shapiro et al., 2007), if HIE adoption is to be successful. A questionnaire survey of emergency physicians concluded HIEs are needed, but most emergency physicians were not aware of the HIE prior to the survey; in addition, the emergency physicians preferred certain types of data and data presentation (Shapiro et al., 2007). Quality of care and safety motivates providers to use HIEs (Rudin et al., 2009), while integrating fragmented health systems will improve quality and decrease costs (Kern et al., 2009). Shapiro et al. (2006) suggest HIEs have the potential to change the standard of care if emergency physicians are responsible for both accessing data in a timely manner and using the information appropriately. In a survey of 216 emergency physicians, the majority reported that access to HIEs would benefit patient care and provide increased efficiency in the delivery of health care (Shapiro et al., 2007).

More studies are needed to assess the data needs of emergency physicians combined with the best methods to implement systems to integrate with workflow (Shapiro et al., 2007). Latency of information, which is the time between the feeder system and appearance in the HIE system, rates of information exchange, and data 
parsing could minimize workflow impediments (Johnson \& Gadd, 2007). Shapiro et al. (2007) reported that HIE adoption is affected by how the data is presented and how accessible the data are to emergency physicians, who indicate a "need for easy and rapid access to information" (p. 703). In the survey of 216 emergency physicians, 54\% reported that patient disposition times (hospital admission or ED discharge) would increase with HIEs because the additional information would require more time to obtain and synthesize (Shapiro et al., 2007). User interfaces must be well designed with emergency physicians involved in the planning and implementation (Shapiro et al., 2007). Altering the workflow of emergency physicians to transition patients from the ED to ambulatory settings or a medical home will be a major societal transition that will require emergency departments to become a reactive settings and ambulatory care settings to become proactive (Frisse \& Holmes, 2007).

The Healthcare Information and Management Systems Society (HIMSS) taskforce reported that usability is the main reason for the slow rate of widespread EHR adoption while the report also identified that the National Research Council had stated that clinical IT systems do not support cognitive tasks and workflows (HIMSS EHR Usability Task Force, 2009). Usability is a system that is easy to use and effective with nine usability principles including: simplicity, end-user comfort, consistency, minimal cognitive load, efficient interactions, use of undo and feedback functions, effective use of language and presentation of information, and preservation of context (HIMSS EHR Usability Task Force, 2009).

An Agency for Healthcare Research and Quality (AHRQ) study reported that EHR usability is subjective and elusive, whereas software features, functions, and interoperability goals are more straightforward (Armijo, McDonnell, \& Werner, 2009). Observation of direct use in clinical settings is required to measure effective usability with attention to workarounds and design that influences errors (Armijo et al., 2009). The AHRQ report outlined EHR usability and information design standards, which included such items as software user interaction, learnability, user control and software flexibility, graphic design, and navigation and exiting features (Armijo et al., 2009). The report also listed specific design features such as adherence to privacy and confidentiality standards and design interfaces that supported rules-based decision-making in a saturated workflow environment (Armijo et al., 2009).

The TIGER initiative, which includes the usability and clinical application design collaborative team, indicated that usability is a human factor consisting of tools, tasks, and environments interacting (Technology Informatics Guiding Education Reform [TIGER], n.d.). The design essentials requiring attention are human factors that include integration with workflow, systems thinking principles, and techniques for data mining with recommendations for ergonomics, standardization, free text, and infrastructure reliability (Technology Informatics Guiding Education Reform [TIGER], n.d.). 


\section{Summary}

This study represents an addition to the growing body of work in the relatively new area of HIE usability surrounding emergency physicians. The literature review identified gaps, such as more qualitative studies are needed to assess the needs of emergency physicians, and to understand HIE capabilities, the environment surrounding HIEs, and usage. HIEs need to be integrated in the emergency physicians' workflow if HIEs are to be successful, but this involves having standardized data available when needed and in the right place, customized features that promote easy access and HIE usability, technical architecture that provides HIE system usability and safety, policies that build a high level of trust in HIEs, unified data standards, demonstrated cost efficiencies, and substantiality.

The issue of emergency physicians using HIEs is drawing attention, particularly since the 787 billion dollar federal economic stimulus package, The American Recovery and Reinvestment Act of 2009, contained several provisions for health information technology, including creating a national information network. Although the NHIN costs are staggering, the United States spent 1.65 trillion dollars on health care in 2003 and costs are increasing by more than five percent annually after inflation (Kaushal et al., 2005). With HIEs having the potential to improve quality and safety of health care, and the potential to reduce costs and medical errors, continued policy efforts will be focused on electronic documentation and sharing patient information across organizations. The emergency departments will be significantly affected by HIEs with the potential to change ED standards of care (Shapiro et al., 2006). This study is both timely and necessary since exploring emergency physicians' usage of HIEs from the point of view of emergency physicians using qualitative research is needed if HIEs are to be successfully integrated into emergency environments. 


\section{CHAPTER 3. METHODOLOGY}

This study was undertaken to elicit physician perspectives that would explore core concepts of how emergency physicians use the health information exchange (HIE) and to generate a theoretical explanation that provided helpful information in the development of appropriate interventions to facilitate physician use of the HIE. The purpose of this qualitative study was to understand the perspectives of emergency physicians concerning the usability of HIEs. This chapter presents the research design, setting, sample and recruitment, instrumentation and procedures for data collection, procedures for protection of human subjects, and data management.

\section{Research Design}

This qualitative study used grounded theory guidance and techniques to gain insights and discover meaning surrounding the phenomena of emergency physicians' use of HIEs in emergency departments (EDs) of selected hospitals in the mid-south. The purpose, desired outcome, and lack of published literature exploring the perspectives of emergency physicians and HIE usability supported using grounded theory methodology to generate a substantive theory. This study explored and explained the usability of HIEs from the perspective of emergency physicians, which was grounded in the emergency physicians' experiences.

Grounded theory methodology is well-suited for broad, open-ended questions, such as the question posed for this research study, how do emergency physicians use the HIE in making clinical decisions? The researcher used past clinical and management experiences in EDs along with accumulated knowledge and an interest in the phenomena of HIE usability and health information technology. The study findings are, "A product of data plus what the researcher brings to the analysis ... Professional experience can enhance sensitivity" (Corbin \& Strauss, 2008). The sensitivity required in grounded theory methodology enabled the researcher, who already was familiar with the topic, to understand the significance of the findings because without some background the ability to both recognize and give meaning to the data would be missing (Corbin \& Strauss, 2008).

Grounded theory methodology, a specific method of qualitative research, originated with Strauss and Glaser in 1967 to generate theory from data (Corbin \& Strauss, 2008), and uses inductive, deductive, and abductive analysis to develop descriptions (conveys ideas about people or things and is the basis for theory), conceptual frameworks, or theory (Charmaz, 2006; Corbin \& Strauss, 2008). The concepts and categories are grounded in the data and developed through theoretical sampling, which is the iterative process of analysis and the sequential collection of data (Charmaz, 2006). Grounded theory methodology is distinguished from other qualitative methods in that the researcher analyzes data while collecting data, an iterative process (Charmaz, 2006). Crucial steps in generating grounded theory consist of initial and focused coding (first 
and second cycle), memo writing (reflecting on the research process and documenting interpretations of findings), constant comparative analysis (comparing incident with incident for similarities and differences), theoretical sampling, and theoretical comparisons (understanding phenomenon through properties and dimensions) (Charmaz, 2006; Corbin \& Strauss, 2008; Roulston, 2010; Saldana, 2009). Grounded theory was particularly applicable as a methodology for this research question because of the strategies for collecting and analyzing data. Morse et al. (2009) suggests grounded theory may be the most commonly used qualitative research method because of "a particular way of thinking about data" (p. 14). The researcher can adapt to the research question, setting, and participants, and describe what is going on and what has happened with and around the phenomena (Morse et al., 2009). The data are synthesized while the researcher develops concepts and theory linked to the data and generalizable to other or future instances (Morse et al., 2009). In this study, transferability (Nigel \& Horrocks, 2010) was used rather than generalizability because readers can gauge if the rich details of the findings and the researcher's interpretations from these ED settings can be transferred to other settings and populations.

\section{Setting}

This study was conducted at four emergency departments located in urban hospitals in the mid-south. The selected study sites (purposive selection) provided a cross section of busy urban emergency departments with access to HIE capabilities. Purposive selection of the study sites provided a partially controlled setting of ensuring that HIE access was available in the EDs. The annual patient volumes of each of the four emergency departments ranged from 36,000 to 60,000 .

All of the 15 face-to-face interviews with emergency physicians were conducted in the EDs or adjacent offices at a time that was convenient to the participants. The participants were given the option to meet at their convenience and at a location of their choice within the hospital. All the interviews were audio recorded with the researcher completing field notes (observational notes) describing the study setting and analytic insights during data collection.

\section{Sample and Recruitment}

The population of interest for this study was emergency physicians who use the HIE. This study initially used purposive sampling followed by theoretical sampling to ensure a wide range of emergency physician perspectives and usability experiences that were developed into concepts with properties, dimensions, and variations and further developed into categories. No exclusion criteria were identified outside the inclusion criteria. Participants meeting the following inclusion criteria were recruited:

1. Emergency physicians that were either full time or part time status and employed in an emergency department in one of the four study sites. 
2. The participant volunteered to participate in the face-to-face interview.

The first two initial interviews were a selected sample of emergency physicians varying in age, gender, and years of emergency experience that were articulate, had a history of using the HIE (either positive or negative experiences), and shared their experiences during the face-to-face interviews. This selected sample was the basis to begin theoretical sampling as concepts were identified during the initial cycle of data coding, and properties, dimensions, and variations of concepts were identified.

Theoretical sampling methods provided an adequate number of emergency physicians who were data-rich participants, meaning they provided depth and breadth in data by identifying how they used the HIE in making clinical decisions along with problems and benefits of the HIE that were developed into themes. The inclusion criteria of only emergency physicians provided rich and credible findings specific to EDs. The specific topic of HIE usability in EDs was the primary focus and easier to define; however, the researcher recognized that posing questions about why emergency physicians were not always using the HIE was a sensitive issue. In addition, topics discussing revenue generation, competition between different health care organizations, and malpractice concerns were also sensitive topics. The majority of the participants (13 of the 15 participants) were articulate, well informed, communicative, and willing to discuss their experiences of using the HIE. However, it should be noted that the other two participants, although not as communicative, provided valuable insights. Sufficient sampling (data saturation/theoretical sufficiency) occurred after 15 interviews, which concluded the recruitment of emergency physicians.

The majority of the participants (12 of the 15 participants) were contacted in advance of the face-to-face interviews either by phone or email to alert them of the study, provide them with the Letter of Introduction (Appendix A), and ask them if they would like to be interviewed. The interview dates and times were scheduled at their convenience after they agreed to be interviewed. Only one of the participants was approached in the ED by the researcher (although identified earlier through theoretical sampling) and two of the participants approached the researcher in the ED. All the participants chose to be interviewed in the ED either during their scheduled shift or immediately before the start of their shift. The interviews were conducted during intervals in the work schedule when the emergency physician was not rushed or had an immediate influx of waiting room patients. In order to collect rich data, the interviews were started and stopped as the emergency physicians attended to patients while ensuring the participants did not experience time constraint pressures. The actual interviews, without the stopping and starting for patient care, took approximately 30 minutes of each participant's time. One participant extended the time to 45 minutes at the request of the participant. Overall, theoretical sampling achieved theoretical sufficiency that accommodated work schedules of the emergency physicians and provided quality (data-rich) participants who were willing to be interviewed in busy EDs. The data was collected over an eight week period.

The recruitment of emergency physicians continued until the researcher determined that the properties, dimensions, and variations of each category were 
sufficiently developed for the purposes of this research (theoretical sufficiency). Theoretical sampling also included sample variation or diversity so that the study included a broad range of behaviors, such as emergency physicians who use HIEs and those who only sporadically use HIEs, and in a variety of situations. Key informants within each ED identified emergency physicians who were considered outliers, such as emergency physicians they described as being generally disgruntled with the ED, or emergency physicians who either openly verbalized they disliked the HIE or others who professed to using the HIE on almost every patient. This provided a diverse sample by interviewing participants with both positive and negative perspectives, and a varied range of HIE usage from occasional to almost every patient.

Since the researcher was considered an outsider by the participants, gaining access required the approval of gatekeepers. The researcher selected the Medical Directors as gatekeepers because of their positions of authority and ability to grant the researcher access to the ED's, but also because many of the participants would consider the Medical Directors trustworthy. The Medical Directors of each study site were an important element in the researcher's success to access the participants. All the Medical Directors expressed interest in the research and how the emergency physicians viewed HIE usability. The researcher provided an overview of the study and the Letter of Introduction to each of the Medical Directors along with the importance of anonymity and confidentiality of participants and minimizing bias through diverse sampling. The importance of not exerting pressure on emergency physicians to participate in the research was stressed. During the interviews, many of the participants denied receiving any information about the study from the Medical Directors so the researcher's view prior to collecting data that endorsement of the study by the Medical Directors would encourage participants to discuss sensitive information, such as how HIEs either contributed or did not contributed to making care decisions, proved to be unfounded. During the interviews, the researcher noted only one participant who may have been coached by some of the responses related to HIE usage rates. None of the participants who were approached by the researcher to participate in the study refused and all the participants completed a single interview, thus the attrition rate was zero.

\section{Instrumentation and Procedures}

The researcher was the instrument for this study and conducted the 15 face-toface interviews. Each interview was audio recorded with the permission of the participant, and transcribed and analyzed using first and second cycle coding by the researcher. The researcher transcribed and analyzed the data to use a hands-on approach to interpreting the data, which immersed the researcher in the data and reflected the ethical decision-making of the researcher's interpretation of the data. "Transcribing interviews is a time-consuming and physically demanding task ... transcription is not merely a technical endeavor... how one choose to transcribe talk involves analytic decisions" (Roulston, 2010, p. 105). Using grounded theory methods, purposive sampling was used to initiate the recruitment of participants followed by theoretical sampling until theoretical sufficiency was determined by the researcher. The study topic and research 
question were derived from the dissertation committee members and the researcher's professional experience along with identifying a gap in the literature. Using the technique of bracketing (Burns \& Grove, 2009), the researcher laid-aside preconceptions and assumptions and focused on the experiences and multiple perspectives of HIE use as told to the researcher by the participants.

The interviews were unstructured and explored the participants' views and experiences using a constructivist approach. Questions were generally open-ended, which allowed participants to express their views freely and prevented forcing data.

Paraphrasing was used throughout the interviews to validate responses. As each interview was completed, the data was analyzed and questions that yielded the best data helped formulated the next questions to fill conceptual gaps. "Grounded theory interviewing differs from much in-depth interviewing because we narrow the range of interview topics to gather specific data for developing our theoretical frameworks as we proceed with conducting the interviews" (Charmaz, 2006, p. 29).

\section{Protection of Human Subjects}

This study was approved by the University of Tennessee Health Science Center Institutional Review Board (Appendix B) followed by approval from each of the four study sites. The University of Tennessee Health Science Center Institutional Review Board, study site one, and study site two approved the study as expedited. Study site three approved this study as exempt and the fourth study site did not require Institutional Review Board approval because patients or patient information was not involved in the study. All four study sites required the researcher to obtain approval from the ED Medical Directors to conduct the study in their EDs. The participants were asked by the researcher to volunteer to be interviewed for this study and The Letter of Introduction served as the participants consent to be interviewed. Written signatures of consent were not obtained because the researcher did not want any identifiers that might compromise the anonymity and confidentiality of the participants. Following review of the Letter of Introduction with each participant, the researcher responded to any questions. All the participants agreed to the researcher audio recording the interviews after the researcher explained the purpose. All transcribed data were number coded with no identifiers to avoid identification of participants. After the researcher transcribed the interviews, the recorded interviews were erased from the digital recorders to protect the identity of participants.

\section{Data Management and Analysis}

The researcher transcribed the interviews using audio-editing transcription software (GearPlayer 4) into Microsoft Word and transported the files into MAXQDA, a qualitative software program. MAXQDA was also used for file management, processing, storage, retrieval, sorting, and reconfiguring data. The interviews were initially transcribed verbatim and double-checked for accuracy by the researcher followed by 
grammatical corrections. All identifiers were removed from the transcribed data, such as hospital identifiers and names the participants mentioned in the interviews. For purposes of readability and clarity, the transcribed interviews were edited by the researcher for presentation and for the audiences the study represents (Roulston, 2010). "While there is much to be learned from transcriptions that include feature of talk such as word stress, pauses, silences, speed of talk, how speakers take turns, and so forth, researchers must be clear on what they want to accomplish in the analysis, and the audiences to whom they will present their work ... Not all studies will require this level [how talk is coconstructed] of transcription" (Roulston, 2010, p. 107). Only the researcher had access to the audio recordings and transcribed interviews. The hands-on method of analysis was used initially followed by coding segments in MAXQDA and searching text to aid data analysis. At completion of the coding and analysis, there were 1132 coded segments and 192 analytic memos. Coding and analysis followed the processes of coding concepts, developing categories, visual mapping, overarching core categories, and identifying emerging themes. Analytic memos encouraged increased levels of abstraction and provided an audit trail. Another literature review was conducted when the interviews and data analysis were completed to compare any new findings from the literature with findings in this study. The literature was used to help identify if the concepts were relevant to this study but not to force concepts (Corbin \& Strauss, 2008).

An audit trail (King \& Horrocks, 2010) provides evidence of the researcher's interpretations emerging from the analysis and is consistent with the concepts and themes developed from the data. The thick descriptions of the perspectives of the emergency physicians show how the themes were developed and how the substantive theory emerged. A coding system was developed in MAXQDA by the researcher and functioned as a codebook, in which codes, a contextual category, were an analytical tool for the systematic analysis of the data (MAXQDA10). Simultaneous coding such as applying more than one code to a segment of text, and the insertion of analytic memos and field notes provided an audit trail. The codes were defined in the MAXQDA code window and assigned definitions and code colors (Appendix $\mathbf{C}$ ). As concepts in the transcribed text were coded, sub-categories, and categories were identified in the coding window. Searches in the text assisted with patterns and relationships as the theory was developed. The assertions made in the findings were supported by exemplars from the transcribed text and substantiated the emerging themes. 


\section{CHAPTER 4. RESULTS}

The purpose of this qualitative study was to understand the perspectives of emergency physicians concerning the usability of health information exchanges. This chapter presents the key results obtained from 15 face-to-face interviews with emergency physicians in urban hospital settings. The interviews were conducted from October to December, 2010.

The findings are discussed using the antecedent condition of HIE usability, which was emergency physicians accessing the HIE during a typical day in the ED, and each of the six themes. The six themes generated from this study were:

1. Using the HIE.

2. Influencing clinical decisions.

3. Struggling with challenges and barriers.

4. Recognizing benefits.

5. Current view of the HIE.

6. Rationalizing not using or reduced-use of the HIE.

The researcher used the thick and rich descriptions provided by the participants in the interviews to enhance understanding the reality of the experiences of emergency physicians using the HIE in daily practice. Exemplar quotations taken from the transcribed interviews represent the participants' perspectives and captured the richness and complexity of the phenomena of HIE usage among emergency physicians. Where appropriate, longer quotations were used to augment and solidify the discussion and help the reader understand the researcher's theoretical interpretation and explanation of the phenomena of HIE usage among emergency physicians. Additional exemplars were presented (Appendix D) to support the researcher's analytic explanation.

The exemplar quotations of the participants are presented in italics and have been edited for readability with grammatical corrections and the omission of utterances (such as, $u h-h u h$ ) and fillers (such as, like, so, you know). Single quotation marks indicated invivo coding, verbatim wording of the participants, or concepts extracted from the transcribed interviews. Brackets are used in the exemplar quotations to denote the researcher's insertion for clarity. Ellipses (...) indicate omitted words or sentences. When words at the beginning of a quoted sentence were omitted, an ellipsis was not used. All identifiers were removed from the transcripts to protect the participants' confidentiality and maintain anonymity as established in the Institutional Review Board study approval. Because the majority of participants were male emergency physicians, the researcher referred to all participants using the masculine gender, he, to maintain anonymity of the participants.

The results emerged from the concepts, sub-categories, and categories and represented the themes to generate the theory. The findings from this study reveal the perspectives of a sample of emergency physicians on the usability of the HIE, including 
how and why emergency physicians use the HIE in busy EDs along with the challenges and barriers they encountered when using the HIE. How emergency physicians used the HIE to make clinical decisions is relevant in clinical practice and patient outcomes.

\section{Antecedent Condition: Typical Day}

Participants described a typical day in the emergency as 'busy' with overcrowded waiting rooms, and patients who are comatose or poor historians with incomplete medication lists, vague histories, unable to provide past test results, or the patients revealed information such as they recently had a CT scan at another facility. There are also patients frequently using EDs who become known as 'frequent-flyers' because of multiple ED visits. Participants reported they did not have a set of criteria for when or why they access the health information exchange (HIE).

We do see a lot of, I can't get in to see my doctor, or with my doctor the appointment is three weeks away.

It's depending [if I use the HIE]. I just look to decide if this looks like it's been recurrent or not. It just depends on what their story looks like.

Some patient comes in, mid 60s, complaining of mid-epigastric pain. She knows she takes medicines for high blood pressure but she doesn't know what the name of it is. She knows she's got, someone told her, that maybe she has some kind of heart disease. She doesn't know what it is, but you go, you talk to her, you try to figure out ... I'm not really sure what she's taking but you gather the history and ... have you ever had a cardiac cath or have you ever had a stress test, or anything like that and they say, well yes, and then you go and you try to describe to them the procedure to see if that kind of prompts their memory of it. They still don't know. So what I usually do is I finish my physical exam, I get as much as I can from the patient, [access the HIE] and see if she pops up in any other hospital, has she ever been there.

Participants reported a variety of reasons why patients visit the ED, varying from true emergencies to non-emergencies. The types of patients presenting in the emergency were described as those with emergent symptoms, unresolved issues, frequent flyers, non-insured, Medicaid, patients avoiding co-pays, patients without primary care providers, or patients unable to access their primary care provider, such as during after hours. Most participants routinely accessed the HIE for comatose patients whether family members were present or not, while other participants did not routinely access the HIE for comatose patients. 


\section{Accessing the HIE during a Typical Day}

Participants reported accessing the HIE to obtain a visit history when patients were using other facilities to receive health services, but the timing of when participants accessed the HIE varied between before or after seeing the patient. However, for some participants timing was not important and they cited convenience or a specific need, such as a patient's visit history, as reasons to look for information in the HIE.

\section{Using Other Facilities}

The most frequent reasons the participants gave for accessing the HIE was when a patient identified 'using' multiple facilities, the most recent visit was elsewhere, or the physician suspected the patient was using other facilities. Patients frequently move between emergencies.

If they tell me that they go [to another hospital] predominately as their institution, then yes, I am going to access them even if they have been in our system before ...It is not a well-defined criteria in my mind, I just play it by ear as to what the patient tells me, what I need to know, and how can I get that information.

\section{Timing or Specific Needs}

The timing of accessing the HIE during the patient encounter was variable with some physicians accessing the HIE either before seeing the patient or after examining the patient, while others only accessed the HIE if they identified a need. The needs varied from providing speedy emergency throughput times, preventing duplicating testing, assessing presenting complaints, or suspicions about motives. Accessing the HIE before seeing the patient was often due to convenience and saving time and not an established practice. The physician may already be in the HIE site looking at another patient, a nurse may have alerted the physician to the patient's visit history, or the physician recognized the patient's name. However, some participants also preferred not to 'bias' their decisionmaking during the patient's assessment and reported they accessed the HIE after examining the patient.

If I think they've been somewhere else, I'm just checking to see or they tell me I had a CT scan done [elsewhere] and that kind of confuses me too. They'll go to one facility, get a work up done, and then they'll decide two days later to go to another facility. That happens very frequently. 


\section{Information Contained in the HIE}

The majority of participants reported that they most frequently accessed the HIE for the patient's diagnostics, discharge summaries, histories and physicals (H\&Ps), and visit history from other hospitals or clinics participating in the community wide information system. During the interviews, participants referred to the community information system as either eHealth or HIE. For clarity of terminology, HIE was used throughout the transcribed interviews. Participants did not discuss the information contained in the HIE without also noting gaps in the standardization of the HIE contents and missing information that was deemed essential.

You may get the medical history from the [managed-care Medicaid program] clinics but you don't get medical histories for anywhere else unless it's in the discharge summary, and they're not all there.

\section{Theme 1: Using the HIE}

The term, health information exchange (HIE), is "the electronic movement of health-related information among organizations according to nationally recognized standards ... EHRs [electronic health records], PHRs [personal health records], and HIE require the use of nationally recognized interoperability standards to enable the flow of information reliably, consistently, accurately, and securely." (The National Alliance for Health Information Report, 2008, p. 22,8). All the participants used the term HIE to denote both the process of EHR exchange and the governing organization supporting the HIE. Although participants did not discuss the nationally recognized standards, they did define the HIE as an information system exchanging patient information.

Participants indicated they did not have an explicit understanding of the criteria for submitting information or what information each facility submitted to the HIE. They were never quite sure what would be available.

[The HIE] varies by institution as to what information is in there. [One hospital] has H\&Ps in there but their discharge summaries maybe 48 to 72 hours delayed getting in there. [Another hospital], they have radiology and lab. I don't think they have any discharge summaries. [Another hospital] has discharge summaries, $H \& P s$, and reports, lab reports. You don't have ED records at other facilities, so it is variable from institution to institution what information is available.

\section{Characteristics of a HIE User}

All of participants indicated they used the HIE, although they recognized the usage varied with some using the HIE more than others. The HIE gives the emergency physician the ability to provide 'better care' for a patient by having access to the patient's medical history. The history enabled the physician to 'better understand' the patient's 
medical presentation in the ED and directs the current course of treatment for an improved 'quality of life' for the patient.

I like the fact that I can pull up records from other hospitals, get more information especially on a patient that maybe says they were just at [another hospital] a couple of days ago. That helps me kind of get more of a background on what's been going on with the patient, especially in terms of not repeating tests, like a patient just got a CT scan for the same presentation two days ago. Whereas, if I don't have access to that I probably would do the CT scan just to be on the safe side. It is also much faster than trying to get records from the medical records department, just what we used to do especially on the weekend or nights ending up being essentially impossible to do. So that I think is very useful from an $E D$ standpoint to be able to say $O K$, I know what's going on with the patient, what has been going on with the patient, is this just the same, is this different, what do I need to repeat, what's already been done.

Without the information contained in the HIE, a physician assesses the patient in a 'blind' state by not having access to important information needed to 'better manage' the patient. HIE information has 'changed the way emergency physicians practice.' They are less likely to miss pertinent information caused by a lack of knowledge about a patient's past test results or treatment interventions.

Somebody comes in with just pain. You ask them if they have had any stress tests in the last six months. You would be surprised how many would say, I have not had a stress test in six months. You go [to the HIE] and they have had a cardiac cath a week or two weeks ago. You ask them, who is your cardiologist? I don't know, it is a foreign doctor or a short doctor. I've had patients come up serious, more than I thought, who have had surgery complications. You ask them, what is the name of your doctor? You go to the HIE labs and 90\% of the time, or 99\% of the time, your doctor does due diligence in order to do that dictation on time.You will often find an op note, procedural note, you find a discharge summary. You will get to know who is the doctor, who's taking care of the patient. Most of the time the patient doesn't even know who their doctor is.

Patients fail to reveal their past medical histories for various reasons, such as they forget important information, or the patient is comatose. Patients are 'not medical professions' and cannot be expected to know what a medical professional considers important information. The HIE removes the 'guess work' for the emergency physician concerning if the patient is revealing everything. The HIE is especially helpful for patients with chronic illnesses, no internal records available, frequent visitors to the emergency, or the motives for the visit are suspicious.

The patient is unable to give reliable information, they have been to other places that have information loaded into the system, and so I access it to gain knowledge about information that is otherwise unattainable or would take days or hours or be very difficult to obtain. The other category of patients I use it in are those that 
have frequently visited the emergency room and there is suspicion about their motives of being here. I am able to find out information that the patient is willingly withholding from me in order to obtain something and in most instances either that means to get drugs here or because they have health care problems that they would otherwise not want to talk about for whatever reason, like drug usage, illicit drug use.

The emergency physician is able to communicate better with patients. Patients may fail to follow recommendations from previous clinical encounters and accessing the HIE allows the emergency physician to assist the patient access avenues to obtain the recommended services or appropriate personnel. Patients with unresolved issues, who are regular users of EDs, will keep going to the emergency seeking help, which contributes to overcrowded EDs.

The ability to access the HIE allows the emergency physician to focus on the current patient encounter and to use the patient's medical history to 'pick up things' that were not there in previous tests. The emergency physician can repeat tests, diagnostics, or treatment interventions if necessary or defer further interventions by using the information in the HIE.

If somebody is here and their back hurts and [the emergency physician accesses the HIE] ... you were at [another hospital] yesterday and you got a script for Percocet. Well you know, you are not getting any more. Or ... if somebody's got belly pain and they had a CT scan, ultrasound, all this stuff done two days ago. You're not probably going to repeat it, that kind of thing.

The HIE is a tool, but two participants contended that emergency physicians should not let the information obtained from the HIE bias their clinical judgment. Both participants reported that the outcome of examining the patient determines if they will access the HIE, which will prevent flawing 'the logical process of decision making.' However, 10 of the 15 participants reported that they accessed the HIE for less than 50\% of the ED patients they examined in a shift, which indicated that many clinical decisions are not impacted by using the HIE (Table 4-1). The majority of ED patients, regardless of their reasons for visiting the ED, received care by physicians who made clinical decisions without accessing the HIE.

[The HIE] is only meant as a tool that can provide information. It doesn't make clinical decisions for you. And if someone has had the workup from here to yonder and you go in and see them and your assessment warrants further investigation, further work-up, then so be it, you do it.

\section{Frequency of Use}

Participants were asked how often they used the HIE in a shift. Accessing the HIE ranged from less than $5 \%$ for four participants to almost on every patient for two 
Table 4-1. HIE Usage Rates $(\mathrm{N}=15)$

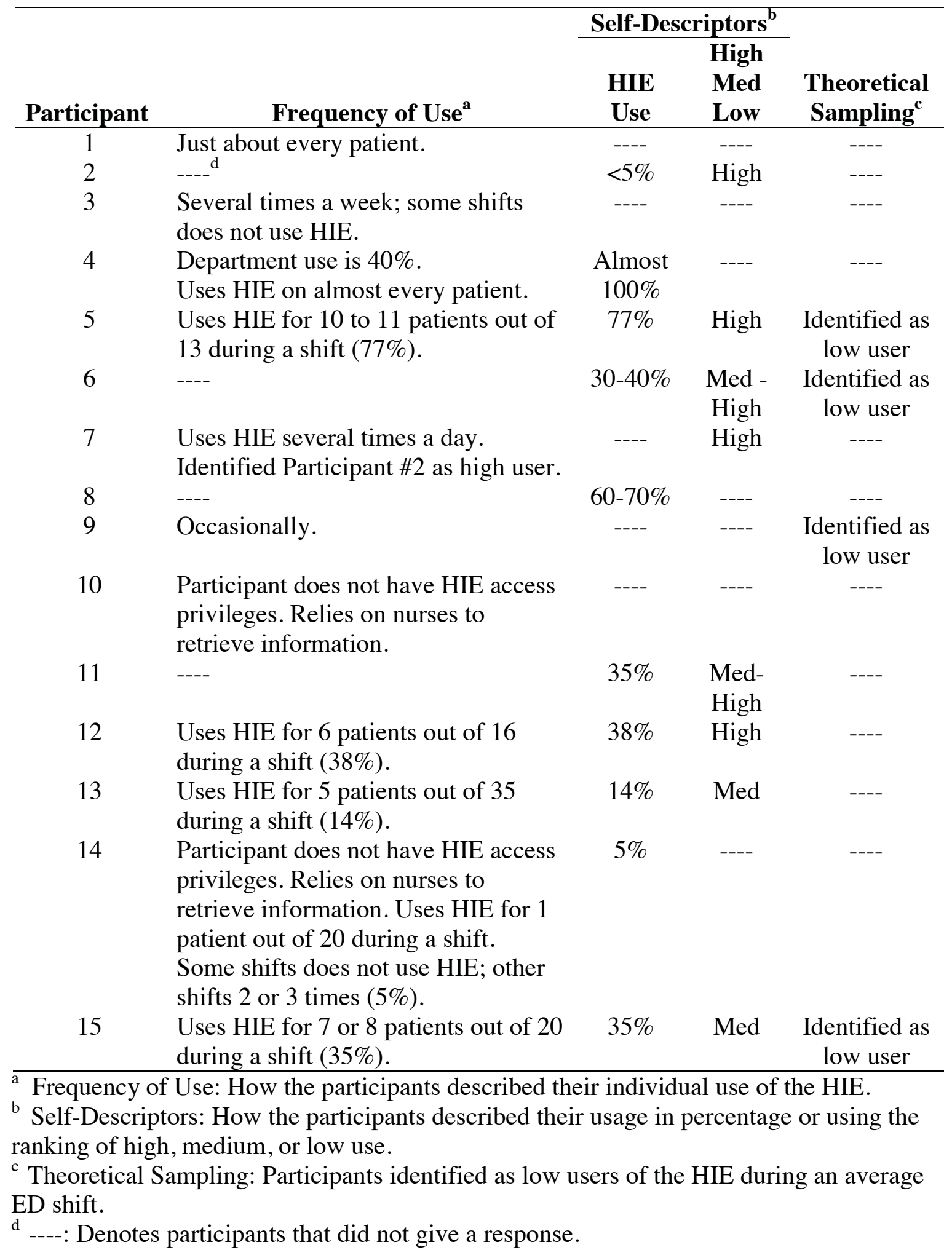


participants (Table 4-1). When participants used the descriptors of high, medium, or low, the percentage of use was inconsistent. One participant identified less than 5\% as highuse compared to other participants who identified medium use as $14 \%$ to $35 \%$. One participant identified his use as high because he accessed the HIE several times a shift; however, this same participant also identified another participant as a high user when in fact that participant stated he accessed the HIE for less than $5 \%$ of the patients during a shift. Using theoretical sampling, four participants were identified as low users $(5,6,9$, 15); however, during the interviews three of the participants $(5,6,15)$ estimated their usage as $30 \%$ to $77 \%$, which was not low usage compared to the overall descriptors provided by the participants. Only one participant identified as a low user described his use of the HIE as 'occasional.' How one physician in the ED describes the HIE usage rate of other physicians in the same department is unreliable and may be in sharp contrast to how individual physicians describe their own self-use. In addition, the physicians had highly variable views as to what percentage constitutes low or high use of the HIE. As a result, when percentages of HIE use is presented in reports or publications, physicians may view single digit results as being high.

\section{Experience Using the HIE}

The HIE was originally implemented in the region in 2006 with resources from federal, state, and local capital investments, and currently links over 450 health care providers in 15 clinic sites and nine major hospitals. Participants indicated that all four study sites implemented the HIE in their emergency departments when the HIE was originally introduced and the HIE system has been in use for over four years. The HIE is managed through a legal entity and governed by a non-profit collaboration of members from health care delivery organization, public health, and state and local governments in a three county area with a combined population of one million. The data are accessed from a centralized database.

Two of the participants have used the HIE for less than one year, while 10 of the participants have used the HIE for four years or more (Table 4-2). Two of the study sites have internal electronic health records with the emergency physicians documenting notes electronically (nine participants). The other two study sites have an EHR system within the hospital but do not have EHRs in the EDs and physician notes are hand written (six participants).

Five of the participants work in hybrid environments, that is, in an ED with electronic documentation, but also in two or more EDs without ED electronic documentation whereby they revert to handwritten physician notes. Of these five participants, three worked in an ED with HIE access, but also worked in two or more EDs without HIE access, and thus, regularly practice emergency medicine without HIE access. However, it should be noted that two of the three participants had not been granted privileges for HIE access at the study site EDs and relied on other physicians, but most often nurses to access the system. When asked if they would miss the HIE, the responses 
were indifferent. The participant, who had HIE access privileges at the study site, 'wished' the other EDs where he worked had HIE access (Table 4-2).

One participant identified that he currently works at two other EDs but accesses the HIE through the internet using his home base login. He reported he 'likes using the HIE,' considers himself a high user, and uses the 'work around' to access the HIE when he is working at other facilities. One of the other facilities where he is employed parttime has HIE access but he considers the access difficult and the other facility does not have HIE access.

Participants reported that the EDs were 'busy.' Two of the facilities are located in the inner city area, while the other two study sites are located outside the city center. The four-study sites reported an annual patient volume in the emergency rooms of between 36,000 and 60,000 with one study site designated a level one-trauma center. Three of the study sites reported that two or three emergency physicians were present in the ED for a 10 or 12-hour shifts (24/7). Only one study site reported that some night shifts have only one physician in attendance.

\section{Who Has Access}

Access to the HIE among emergency staff varied within the study sites. The majority of participants expressed confusion as to who had access both within the ED and outside the department. The study sites were not consistent in which personnel within the EDs or physicians within other areas of the hospital had access to the HIE. Within one site, one participant identified that registered nurses were not authorized for access, while another participant in the same site indicated that charge nurses and maybe the nurses

Table 4-2. Participant Profiles $(\mathrm{N}=15)$

\begin{tabular}{lcc}
\hline \multicolumn{1}{c}{ Environment and Experience } & $\begin{array}{c}\text { Study } \\
\text { Sites }\end{array}$ & Participants \\
\hline Electronic Documentation in ED & 2 & 9 \\
Electronic Documentation NOT in ED & 2 & 6 \\
Hybrid Systems ${ }^{\text {a }}$ & & 5 \\
$\quad$ With and without ED electronic documentation & & 3 \\
$\quad$ With and without HIE access & & 2 \\
HIE Experience- Years of Use: & & 3 \\
$\quad$ Less than 1 year & & 10 \\
1 to 3 years & & \\
4 or more years & & \\
\hline
\end{tabular}

${ }^{a}$ Hybrid systems denoted participants who work shifts at more than one hospital with varying levels of HIE access and/or electronic documentation. Some hospitals have electronic documentation and others do not have electronic documentation, or, some hospitals have HIE access in the ED and other hospitals do not have HIE access. 
assigned to patient registration had access. In this same site, medical students and residents did not have access. The residents would come to the ED and ask the emergency physician to log into the HIE to obtain patient information. Some of the physicians on the units had access but the participant indicated they seldom used the HIE because they relied on residents to obtain the information from the medical record departments.

Two of the sites identified that chest pain nurses had access and would pull the HIE information for the physicians, print the information, and place it on the clipboard. Within one of the study sites, nurse practitioners, physician assistants, and any nurse who wanted access to the HIE could submit the paperwork. One participant stated that nurses did not want access and he did not see a benefit to nurses having HIE access; furthermore, he did not know who had access. However, another participant at the same site, who did not have HIE access privileges indicated that he accessed the HIE through the nurses who would look up information and alert him if pertinent information was available. The information was printed and placed on the clipboard. Again, the process was not clear to the participant because he thought printing of some information was restricted and left the decision to the nurses.

[The nurses] print off what they feel like would be pertinent to the most recent discharge summary, or the visit histories and stuff like that. There's certain parts of it that are not suppose to become part of the medical records so they always tell me you can't keep this part, just look at and chuck it. I don't even know.

Another site with a participant who did not have HIE access privileges also relied on the nurses to access the patient information. Other participants in the same site relied on nurses for printed information although they did not know exactly who had access and the process of printing off information was sporadic.

Now I know the charge nurses do because they sometimes will go print some stuff off for us as well. I believe that they do, but I do not know to what degree or what extent.

Scribes, who act as personal assistants for the emergency physicians, were used at two of the study sites. One participant described the scribe's function as accompanying the emergency physician and 'doing all the computer documentation' or most of it. The scribes at one site had access to the HIE in addition to the nurse practitioners and physician assistants; however, ED nurses, residents, consultants, or physicians on the units did not have access. The participant indicated the ED Director was advocating for policy expansion, which would allow the physicians outside the ED to have HIE access. Because consultants did not have privileges to access the HIE, the participant contended duplicative ordering would remain problematic. 


\section{Theme 2: Influencing Clinical Decisions}

All of the participants verbalized that the HIE influences clinical decisions but the opinions ranged from the information 'changes the way emergency physicians practice' to 'it does not have a huge impact.' The majority of participants implied that the more information available to the physician, the 'better the decision-making.'

I think [the HIE] has a tremendous capability to improve patient care and prevent patient injury, like from repeated radiation or multiple frivolous work-ups that are just repeated over and over.

Other participants communicated that the HIE did not have a notable impact on influencing their clinical decisions. One participant indicated that the information from the HIE could be important in decision making, but time constraints and the busy pace of the ED was more of a concern than taking the time to look up the information.

Well, I think of the times that I go to [the HIE] because I go to it quite abit, it's only helpful probably one out of five times that I use it ... because I am going to it to see if there is something there that will change what I will do today. The times that it actually changes what I do is very slim just by definition because a lot of times a patient has not been seen by hospitals that have data there, or they haven't had the tests I am looking for. It actually changes what I do very seldom.

\section{Missed Information}

Because emergency physicians rely on the information patients can give at the time of the encounter, participants expressed that they miss 'a lot of the patient's problems' without access to the HIE. The ability to 'pick-up' information that is not readily evident during the current visit helps the physician piece together a clinical picture that influences the clinical course.

A patient I had seen prior, I had actually performed her catheterization at [another ED where I work], and she looked familiar, but didn't kinda strike up. But once I saw my cath report, I knew exactly what was going on so I was able to get her out of the ED and get her home. So it definitely changed my opinions at that time.

\section{Assortment of Clinical Decisions}

Participants reported an assortment of clinical decisions influenced by the HIE ranging from identifying allergies, assessing if the presenting problem is new or has changed, previous work-ups, ED visit history, or providing clues about current medications, especially for poor historians, comatose patients, patients with chronic illnesses, or patients who give unreliable information, such as drug seekers. 
You know one other major issue-people that go to ERs frequently, drug seeking, and that sort of thing. That's another huge advantage there. You can look and see if they've had workups, if they got other medications elsewhere, kinda streamlines things, which gives me an idea what their true motives are.

\section{Tracking, Comparing, Trending}

The HIE allowed physicians to track test results, compare abnormal results with previous results, or detect trends that alter clinical decisions, such as changing the patient's disposition, which saved time in the ED for both the physicians and patients. Participants viewed saving time and avoiding duplicative ordering as cost savings for the overall healthcare system.

If I see a lab ED, his liver enzymes are always elevated for the last six months and I am worried about it right now. But if I have no access to that and if I see liver enzymes are elevated, I would have to do something about it. [The HIE] helps me out.

Other participants saw a need to refine the processes, provide trending graphs, and make the HIE easier to access the information. Participants reported both a lack of understanding how the HIE system functioned and differences in the functions available within the HIE system. Some participants reported they used the graphing function, other participants reported there was no capability within the HIE system to provide graphs while other participants reported the graphing function only worked intermittently.

A lot of times I am looking for is a trend. Their blood count was this a week ago, what is it now. And that can be difficult sometimes if the patient, let's say had two weeks of hospitalization at [another site], kind of sorting through, OK, what exactly was done. The other is I wish that more were like [names hospital site], I can pull up the records there but there is more of a discharge summary. There is more of the inpatient course included because a lot of times you'll see three weeks' worth of labs but nothing about what was going on with the patient in terms of what the consultant was thinking. Did they have EGB done by the GI doctor? So importing more of the kind of procedure operative notes in there would also make it more useful though it is still extraordinarily useful as is.

\section{Reaction Time}

Using the HIE changes what the emergency physician is going to do for the patient and 'how fast' the physician needs to act. In addition, participants reported that the information provided in the HIE is valuable for patient transfers and saves the physician time by not ordering repeat diagnostics. 
[The HIE has] made it easier for us to find out studies that have been done on patients. It's easier for us to find that and reference current lab values to previous lab values. It changes what you are going to do for a patient. You can tell if a patient is chronically sick from that or they're now sick, and then before they're not sick. It tells you how fast you need to act on a patient.

Other participants reported they did not use the HIE and reaction time was not a factor in their decisions. The EMR frequently did not contain pertinent information or the medical opinions of the transferring physicians did not influence their management of the patient.

I mean there is like six pages [in the electronic discharge summary]. You have to read through all of it and maybe $20 \%$ is valuable information and the rest of it is just junk.

I think that there are times that I don't necessarily care what the outside hospital's doc was thinking like for transfers. That OK, they are here now and I'm going to get a fresh set of eyes because I may not trust what I have been told over the phone. I don't think though that having access to the information influences you in a negative way. You can choose to be like, well I understand they had a CT scan yesterday but this pain is different, or they look horrible right now and maybe they have something that the CT didn't show before. So I don't think you are ever hamstrung by, well they just did this yesterday. We see plenty of people that come in the day after or two days after they were seen in our ED.

\section{Amounts of Radiation}

Imaging and the amount of radiation patients receive was a reoccurring theme in how the HIE influences clinical decisions. Overall, 10 of the 15 participants articulated that the HIE, as an information tool, has reduced the overall number of CTs; however, the reduction of diagnostics was variable and was influenced by the physician's practice behavior.

But a patient has abdominal pain, has headaches, stuff that I know he's had before, then I will take a look and see what has been done for it. If nothing has been done in awhile, then we'll see if we need to retest. But if I saw that he was in the hospital two days ago for the same thing and they did this, this, this and that, then I don't need to repeat it. I don't need to repeat, I don't need to radiate you anymore and cause more radiation.

Four participants expressed reservations. The range varied from one participant inclined to work the patient from 'scratch' compared to another participant who focused on only the immediate complaint. The subject of each facility preferring their own studies was expressed with the notion that reports cannot replace images, particularly for 
consultants. Three of the participants agreed that the HIE does make a difference in their practice but not to any great degree.

Somebody comes in with abdominal pain that was seen [at another hospital] yesterday, had a negative CT scan, we're still going to scan them because the consultant wants to have that information and wants to look through it. Now, the more interesting question is, if the consultant had access to the images, not the report, would we reduce the radiation and I think the answer is $100 \%$ yes. But right now, the answer is no. [The HIE] has not and probably will not because until that is the case you won't change anything. I will say that is just for CT scans. There have been times where I have said, this patient just had a CT scan at [another facility] and had a CT scan of the head and just had it yesterday. I'm not going to rescan them. I will document on the medical record that I reviewed the results of the CT scan from the medical record from [HIE].

\section{Ordering Tests}

The ability of the HIE to influence other clinical decisions varied. Other tests, such as laboratory, were not viewed 'as critical' and participants were inclined to repeat the tests because 'lab values tend to change more.'

The blood work is pretty even. If the patient was there five days ago, it's a new thing so we still have to do it. I am talking about a CT scan or procedure, which could have been avoided because they looked it up.

The majority of participants described patient encounters whereby accessing the HIE reduced the overall number of diagnostics ordered, particularly preventing duplicate ordering. However, the emergency physician's practice behavior influenced the decision to order repeat diagnostics. Specific examples revealed that the HIE may or may not influence whether patients with cardiac symptoms would have repeat diagnostics.

Specifically with abdominal pain that has a recent CT scan of the belly. That is a very common one. I don't think it has cut down on chest CTs. Probably doesn't cut down on cardiac imaging just because chest pain is such a high risk diagnosis that regardless of what tests they have last week, you are still going to approach them today from scratch almost. It is hard to say last week they were fine, so today they must be fine as well.

\section{Concept of Life-Saving}

One participant expressed that the influence of the HIE has 'evolved over time to be not only an invaluable tool but a life saving tool.' However, this same participant also verbalized that during chart reviews he had never seen a negative patient outcome 
because the HIE had not been accessed during the course of the patient's encounter in the ED.

When somebody presents with a heart attack, the ambulance takes those patients to the nearest hospital. It may not necessarily be that patient's hospital. That patient may be having a heart attack ... But that doctor has to have access to all the information here in order to be able to say, where is the patient's last cath? What's been going on with the patient? Although the history from the family is great, we need more than that as physicians. We need to know the specifics of what is going on ... But to be able to have that information at your fingertips right away that can be lifesaving. And it has been.

\section{Theme 3: Struggling with Challenges and Barriers}

All of the participants cited multiple challenges and barriers when using the HIE that either restricted their use or deterred increased use. They were passionate in verbal expressions that the HIE was not as 'user-friendly' as they would like and the term 'cumbersome' was frequently used to refer to the HIE.

I have to sign in every time that I need access is very cumbersome and it is not conducive to the smooth flow in operations and expediting flow through the emergency room.

The challenges and barriers to using the HIE were collated into six categories:

1. Difficulty accessing the HIE.

2. Granting privileges to access the HIE.

3. Gaps in HIE information.

4. HIE design flaws.

5. Emergency Physician communication: between physicians and between physicians and HIE personnel.

6. Interoperability of HIE.

\section{Difficult to Access}

Participants identified a need for easy access to the HIE with the minimal number of 'clicks' to provide speed in obtaining information and accommodating the fast-paced workflows inherent in busy urban EDs. The majority of participants expressed that there should only be one login with password entry into an integrated system that would provide the physician with access to the HIE, State prescription site, internal hospital EHR system, or other necessary icons. Each additional step of repeated logins with password entry increased the number of 'clicks' and restricted workflow, which is deemed not conducive to the practice of emergency medicine. Frustration was expressed over the multiple times a physician had to login to access the HIE. 
[The HIE is] an extra step and it's hard to do, there's really no place. It's one extra click in a computer, [which] is painful for an emergency doctor and you're talking minimum three to four clicks here to get in.

A number of participants remarked that access improved from the initial implementation of the HIE. Difficulties were encountered when keys (also referred to as tokens, fobs, or badges) were used since the keys were lost or misplaced and the physician either did not use the HIE for the shift, or 'borrowed somebody else's, or you had to have them login.' Participants viewed the introduction of single sign-on as an improvement that increased HIE use, although many participants continued to express displeasure that accessing the HIE requires a separate login and password entry each time the HIE is used. Two study sites that had electronic documentation within the ED maintained that single sign-on for the HIE was not enough to provide efficiency. Participants argued that the HIE and EHR should be integrated in order to have one signin for both systems, and not the current system of a separate sign-in for the EHR followed by another sign-in for the HIE.

I would like to have [the HIE] integrated where I just have to hit one button and boom, I've got it. Because when I have to go through two or three steps to get into it, I'm not going to do. I have just got too many people to see, I've got to keep rolling and I'm not going to look at [the HIE].

One study site, with an EHR system within the hospital, has put considerable effort into streamlining HIE access in preparation for the EHR implementation in the ED. The participant viewed the effort to decrease the number of clicks and provide seamless entry with the EHR as being an essential element to increase HIE use among physicians and prevent failure of the HIE. Overall, participants from this study site stated that the HIE access 'was easy;' however, the department had not implemented electronic documentation at the time of the interviews and the physicians were handwriting the emergency physician notes.

\section{Privileges and Access}

The process of acquiring access to the HIE involved emergency physicians receiving a login and password. Two of the participants had not been granted HIE access, and yet they worked regular shifts in the ED. Both participants stated they did not have access because they found the paperwork process laborious. Another participant reported he did not receive access for two months after starting employment in the ED. In conversing with another physician who was not a participant, but working regular shifts in the emergency, he revealed that he did not have access.

I signed up with the people for a password or whatever, but it was problematic. I tried putting in the codes and all that and then I was supposed to call back and call somebody else, so finally I just got tired of messing with the email and phone calls and decided not to worry about [the HIE]...When I called back I got a voice 
mail, so rather than get aggravated about it I just let the charge nurse look up whenever somebody I have a question about.

The process of granting privileges was variable among the four-study sites, which caused inconsistencies among staff members as to who had HIE access both within one ED and across the study sites. For example, nurses were not granted privileges in two of the study sites. The majority of participants could only guess who had been granted privileges with some hospitals granting privileges to nurses, residents, scribes, and/or some hospital physicians while other hospitals restricted access to emergency physicians, nurse practitioners, physician assistants, and scribes. Some of the participants viewed that their time was being used to provide printed copies of HIE reports for hospitalists, consultants, or residents who were denied privileges to access the HIE.

Emergency physicians who had not been granted HIE privileges had to find another physician to access the HIE or a nurse who had privileges to print information. Printing material from the HIE occurred on a regular basis in all four study sites and the process for handling the printed material was not understood by many participants. Both participants who had been granted privileges to access the HIE and participants who did not have access printed material from the HIE, or had nurses or residents print material. Participants reported they were not fully aware of the process for disposition of the printed material, but conveyed that the material was shredded after use, attached to paper medical records, or given to hospitalists or residents.

\section{Gaps in Information}

All of the participants indicated there were gaps in the HIE information. Various participants viewed accessing the HIE as a waste of time if gaps occurred in the HIE content, commonly due to inconsistent data entry, delays in entry, and lack of content standardization. All the needed information was 'almost never' available. Participants were not aware of what each hospital was committed to entering in the HIE database. For example, the emergency physician notes were present only if the hospital had EHRs implemented in the ED, but not all participants were aware of this process and stated that the report had not been entered into the database. In addition, not all the hospitals in the catchment area had the HIE; thus, gaps occur in the patient's medical history. Participants expressed a desire to have the HIE encompass the entire catchment area including hospitals, clinics, physician offices, and pharmacies. A couple of participants expressed a desire to have a nationwide HIE.

\section{Design Flaws}

The most cited HIE design complaint was the HIE logging out too quickly. Participants could not identify exactly how long the HIE would stay open for access, but some estimated 15 minutes. This contrasted with the EHR remaining open longer. Participants failed to understand how the HIE information security varied from the EHR 
security. All four study sites had physician documentation areas with computers designated for physician use; however, the computers were shared with nurse practitioners and physician assistants and open to the flow of ED staff traffic. The EDs using scribes also accessed the same computers as the emergency physicians. However, two participants, one from an HIE emergency site with electronic documentation and the other participant handwriting emergency physician notes, did not have an issue with the time limits of the HIE. Additional design flaws identified by participants were the HIE screen is too small, there is too much scrolling, the visit history does not identify the reason for the visit, and retrieving laboratory values is problematic. As a result, obtaining HIE information is too slow.

Yes, I wish I could keep [the HIE] up. That's the problem. I hate having to sign in ... Well that is not conducive to emergency medicine ... I'm gone for a matter of minutes and I'm coming back. I have to sign in every time that I need access is very cumbersome and it is not conducive to the smooth flow in operations and expediting flow through the emergency room.

\section{Physician Communication}

Using the HIE is viewed by the participants as more challenging when the design of the HIE 'lacked' emergency physician input and the physicians were not afforded the opportunity to provide regular feedback about barriers they encountered when using the HIE. The use of the HIE is more challenging when education or hands-on training sessions of HIE features are not offered to the emergency physicians on a regular basis. Participants reported learning how to use the HIE or find information on 'their own' and as a result they were not fully aware of all the HIE features or the most expedient steps to access the information they were seeking. This may contribute to participants reporting that the HIE is 'cumbersome to use.' Notification of updates would be beneficial in keeping the end-users informed of changes especially since participants denied discussing the HIE with other emergency physicians within their own hospital or other hospitals in the city. Some participants reported that as new emergency physicians in the department they 'heard almost nothing about the HIE.' The majority of participants stated that they neither had contact with personnel who managed the regional HIE system nor did they know who to contact. As a result, participants stated that the HIE was not as 'userfriendly' as they would like and 'it is a slow process,' which are deterrents to usage. The majority of participants had encountered problems with physician communication.

Physician input and feedback. Participants identified a need to provide input into the both the design and function of the HIE to make the system more user-friendly. In addition, participants contend that being apprised of how to give feedback would provide the outreach needed to identify and correct barriers and challenges to using the HIE. One participant, who indicted he had never been asked to provide feedback, viewed that giving input might have negative consequences, such as slowing down the speed of 
accessing HIE information. As a result, he would likely decline to give feedback even if he was asked by HIE personnel.

Education and training sessions. Participants stated that education was an essential element to the success of the HIE system. The majority of participants identified deficits in receiving hands-on training that would facilitate easier navigation of the HIE and might promote increased HIE usage. The majority of the participants denied receiving any education or hands-on training sessions on the function or operations of the HIE.

One participant identified that the problem was not a lack of education by the HIE system but by the hospitals in the HIE database; however, the issue was the participant had not been made aware of what each hospital in the HIE database could contribute based on the functions of the HIE. The participant stated a particular hospital did not provide ED notes, but the identified hospital did not have electronic documentation in the emergency and therefore had never put ED notes in the HIE because scanned notes are not accepted by the HIE database. Other participants also expressed confusion as to what reports were in the HIE.

Only one participant verbalized being a strong advocate and early-adopter of the HIE and identified that there were new HIE versions about every six months. He stated that education of the HIE was lacking in other hospitals but not in their ED. The participant described that their department had a mechanism in place for the emergency physicians to receive education and updates while providing feedback. This participant identified the process as essential for the HIE to be used.

Receiving updates. Although one participant had identified that there were new HIE versions about every six months, most participants reported they were not aware of any changes in the HIE other than the early change from fobs or keys for accessing the HIE to entering a userid and password, which was described by some participants as single sign-on. One participant reported that discharge summaries had been added to the HIE database about two years ago. Participants who stated they received updates were from the two study sites that did not have electronic documentation in their EDs whereas participants at the two study sites with electronic documentation denied any regular contact or recent updates in the system.

Contact with HIE personnel. Most participants reported minimal or no contact with personnel who implemented or manage the HIE. Usage rates had been provided in the early phases of implementation but participants stated they no longer receive any reports or information and are not aware of current use. Participants did not seem interested in usage rates. Only the one participant who stated he received updated HIE versions was aware of the department usage rate for his emergency. 
No feedback to us at all. Probably does occur. I would not be surprised if it did ... I don't get a letter saying yes, you used the HIE 46 times last month. No.

The one participant at a study site without electronic documentation in the emergency who reported he received updated HIE versions every six months also stated he had regular contact with HIE management whereby he provided feedback. He reported that the HIE utilization for their department was $40 \%$. Overall, this participant reported open communication with HIE management on a friendship basis and contended that he had input into the HIE system. This same participant considered he was a key player in the function and success of the HIE in the region. This participant was in contrast to other participants who did not view receiving HIE information or providing orientation for new physicians in the ED as important. Other participants described learning about the HIE and the functions on their own.

Communication between emergency physicians. The majority of participants stated they have not discussed the HIE with other colleague within their own department or with emergency physicians in other EDs in the city and as a result they are generally unaware of HIE activity within the region. Participants that identified difficulties in navigating the system or had questions could not identify a source to help answer their questions.

\section{Interoperability}

Interoperability is, "the ability of different information technology systems and software applications to communicate, to exchange data accurately, effectively and consistently, and to use the information that has been changed" (The National Alliance for Health Information Technology, 2008, p. 10). Participants spoke of interoperability problems with the HIE on a broader level to include the EHR, such as connectivity failures or the inability to obtain data the physicians proposed was necessary. For two of the study sites not yet using electronic documentation within the ED, participants expressed issues with the EHRs from other facilities or within their own hospital, such as inaccurate and incomplete information and lost productivity.

It's a little bit difficult to actually get in a direct link into the medical record and there is proprietary problems associated with the software that they are working with.

Our EMR is kind of cumbersome. It is hard to read to find out what happened ... You have this paper chart [pointing to clipboard]. You go there [to patient], you make your note, you come back and you document. It's wasting our time. They have this tablet, which is not functional use. You can take that in the room, it's not working. 
Interoperability gaps in the HIE were identified in:

1. The ability of systems and software applications to communicate.

2. Lack of standardization in exchanged data.

3. Failure to accommodate information needs of emergency physicians.

4. Inability to access information in patient rooms within the ED.

5. Hybrid work environments whereby the physicians were alternating between EDs with HIE access and department without HIE access.

System and software communication. How the systems communicate and the software requirements were not understood. Generally, participants assumed a function or expressed need could not be operationalized, such as wanting to provide more information in the discharge summaries, but they never received an explanation if the need or desire could be fulfilled. Other participants reported that discharge summaries from some facilities were incomplete. One participant expressed frustration when he received an explanation from senior administration that their internal EMR was the 'wrong format' to communicate with the HIE system.

Standardization. Standardization was viewed as receiving consistent information with consistent presentation within a designated time frame from all the facilities. None of the participants freely expressed that the HIE was standardized but rather articulated examples of inconsistency. Reports that were deemed essential, such as discharge summaries or clinic information are frequently missing. Many participants had never been informed what information was available from each facility in the HIE database or why the presentation of reports, such as radiology reports, were not consistent between facilities.

[One hospital] doesn't categorize their x-rays in [the HIE], all they show is for instance, if you want to look at an $x$-ray from [that site], all it says is [site name] something, but it doesn't tell you what kind of $x$-ray it is. It doesn't differentiate between a CT scan, an ankle $x$-ray, or chest $x$-ray. And that makes it very, very difficult to see what is going on ... You have to go through every single $x$-ray because they are not categorized as to what kind of $x$-ray they are in order to find the one that you want. [The HIE] doesn't categorize them as CT scans or what kind of x-ray of the neck or the ankle. You gotta kind of guess. There might be ten $x$-rays and the one you are looking for might be the last one on the list but you gotta go through all of them to get to the one you want.

Although one participant 'thinks the way [the HIE] is presented is consistent,' he noted inconsistent levels of completeness. The participant stated that he 'has seen' discharge summaries from all the facilities in the HIE database, but the level of completeness of documentation was inconsistent and related the issue to physician documentation standards. 
Almost half the participants viewed the difficulties as hospital problems and not an HIE interoperability issue. Participants reported a lack of criteria standardizing HIE content and inconsistent time frames for data submission. Some participants also did not know which facilities were not using electronic documentation in the emergency and thus, emergency physician notes were never available in the HIE from these EDs. Participants expected to see the notes when using the HIE and reported this as a variation in data. Other participants were aware that scanned documents could not be submitted to the HIE database and viewed this as an interoperability problem of the systems.

That is nothing to do with them [the HIE]. They can't do anything about that. It is from the hospitals ... I would like to see it but I don't think it's possible ... Yes, the ideal would be able to see everything.

Information needs. Information needs ranged from the HIE not meeting needs to a 'wish list.' The most frequently reported need was including EKG tracings in the HIE. Participants related that being able to compare previous EKG tracing with the current presenting symptoms and EKG tracing is a priority need. The majority of participants stated the HIE system could not provide the tracings because of a lack of interoperability functions. Other cited deficits in the HIE information included inconsistent availability of reports such as emergency physician notes, discharge summaries, outpatient clinic reports, and consultant reports. Because the HIE presents screen shots of laboratory values, participants identified a need for graphing capabilities to allow trending and reduce the physician's time to view values from individual patient visits. However, some participants identified that graphing capabilities were available but not always functional.

They still don't have the capacity to put pictures of old EKGs and that's a very important thing to us. I have talked to them a bunch of times. They are trying to work it in but it takes so much memory, pictures versus text. I can see the text, what the computer describes it but actually can't see the images. But other than that most of them will have nearly everything.

Another view expressed by participants was the streamlining of the HIE and a minuscule approach to the amount of information that was necessary to preserve speed within the system. Increasing the information was seen by some participants as unnecessary for emergency medicine and would slow down the speed of the HIE when accessing information. Speed was frequently cited as a priority in emergency medicine. However, one participant stated he did get enough information even though he had to 'piece' the information together without medication histories or clinic notes.

Connectivity and equipment. While some participants stated they had enough computers in the department, other participants reported a multitude of problems other than the integer number of computers in the department. Connectivity entailed the ability to access the HIE throughout the ED, including examination rooms, but with seamless effort to accommodate workflow and no loss of speed retrieving information. Equipment 
needs involved discussing the configuration of the physical structure of the ED to provide easy computer access to the HIE, facilitate the use of portable devices to access the HIE at the bedside during patient encounters, or if there were enough computers in the department to prompt easy use of the HIE.

Portable devices were viewed as non-functional within the current EDs. Participants reported connectivity issues, slow function, cumbersome devices, or a change in practice behavior such as using computers while examining patients. Participants indicted that connectivity or the ability to use portable devices at the beside was a barrier. One participant identified that he also worked in an ED that had portable devices, but he did not use the portable devices. Other participants at the same site reported they rarely used the portable devices because of poor function.

I'd like to see [the HIE] used on an iPad. I would like to see it integrated into portable computers, mobile website ... So I wouldn't have to come over here [to the documentation area in the ED], but do it on the run. The department is all around.

The question if the department contained enough computers to access the HIE was variable and ranged from enough computers to not enough. One participant indicated there were enough computers in the department but the computer stations were not convenient due to the physical layout of the ED. For one participant working shifts in more than one ED, the variation in the number of accessible computers was noticeable. A small number of participants indicated that more computers available to the physicians would ease access issues and maybe promote more use of the HIE.

Hybrid work environments. For participants working in more than one ED, they encountered hospitals with or without access to the HIE and a variety of EHR systems. This created hybrid practice habits whereby participants adjusted to making clinical decisions without the benefit of the HIE. Although participants working in hybrid environments verbalized that the HIE was beneficial, one of the participants proclaimed, 'and they don't have [the HIE] and I wish they did;' however, none of the participants stated that the absence of the HIE altered their decision making practices when compared to having access to the HIE. One participant had only been using the HIE for four months and indicted the HIE was 'a luxury, very, very helpful.'

One participant worked shifts in three different EDs and was unaware that one of the EDs had HIE access. However, he maintained access to the HIE with a work around.

I don't think we have access there. Maybe they are on the system but I am a part timer there so I don't know much about it ... I never used [the HIE] from there.

Not all EHR experiences are positive with participants commenting on their displeasure with electronic documentation, particularly the length and inaccuracies of EHR reports. However, for some participants their negative opinions of the EHR did not 
influence their use of the HIE. Participants at one site reported they did not like electronic documentation and were 'not looking forward' to the EHR implementation in the ED in the near future. One participant verbalized his displeasure with using the EHR, but indicated he used the HIE $35 \%$ of the time and found the HIE very helpful.

I work the same at [another hospital with an EHR], and it takes me, to do a case, 10 minutes on a single chart and here it takes me a minute or two maximum to do a T- sheet. So I think it is a huge, huge difference. Doing manually is so much quicker than doing the computer. There is no question about that. I am a good candidate for your [interview] because I work at two different places because they have a computer there [ED electronic documentation] and we don't have it here ... The product, every product is different ... Writing is much quicker.

The other is that the danger with the electronic records to just check everything, check nothing, so you have patients with mechanical heart values that have no murmurs and patient who have been hemiplegic for the last 20 years move all four extremities and so that is not uncommon.

\section{Theme 4: Recognizing the Benefits and Success Factors}

All the participants reported benefits of using the HIE, including participants who were considered low users within the department. One participant used the analogy of three pillars to describe the benefits and successes of the HIE by improving patient care and safety, increasing efficiency, and realizing cost saving.

\section{Avoiding Duplicative Testing and Unnecessary Ordering}

All the participants prioritized avoiding duplicative testing and omitting unnecessary ordering as a benefit of using the HIE, which results in achieving the three pillars. However, the levels of avoidance varied from substantial to minimal. The majority of participants stated they avoid duplicative ordering of CT scans. Lowering the number of CTs an individual receives improves patient safety by reducing unnecessary exposure to radiation, particularly when duplicative orders can be eliminated. However, two of the participants stated that the HIE did not reduce their practice of ordering CT scans, while one participant declared the reduction was minimal.

They have had enough radiation to glow in the dark ... there does become a point where [the patient] had six negative CT scans in the last month, enough is enough.

When discussing the reduction in diagnostics, other than CT scans, participants reported a decrease; however, their responses were not as explicit related to laboratory testing. Participants had difficulty estimating the number of tests avoided or not duplicated. Some participants claimed that the reduction in the overall number of 
diagnostics is not significant. Participants frequently viewed that averting the ordering of laboratory tests was 'not as critical' since the values 'tend to change more' and thus, required repeat ordering. However, participants generally proposed that patient care is improved by only providing what is necessary for treatment and eliminating not only duplicate ordering of CTs but also other diagnostics.

Radiation, yes. We definitely don't do as many as we would have, or X-rays, because of [the HIE]. I mean we just don't, because if you know that they have had one, well, you had this for back pain ... Lab tests also, and stress tests and caths. Everything is decreased because of it. It saves money, because if you know that they just had a cardiac cath at another hospital a month ago, you're not going to repeat it.

For me, [the HIE] minimizes me repeating tests, makes me get a patient to the right doctor, make the right decision efficiently.

\section{Efficiency in Patient Flow}

Moving patients through the ED faster benefits the patient and improves time efficiency so that emergency physicians can see more patients. Cost savings for the ED were reported when physicians avoided duplicative ordering, but some participants also reported that as a result of omitting duplicative ordering the physician saw more patients.

One participant reported that the HIE frequently did not reduce the number of diagnostics ordered, but the information does change the disposition of the patient and influences the time the patient spends in the ED. ED visits are decreased when patients are managed more efficiently. Participants reported that patients who frequently visit EDs can be referred back to their primary care provider and drug seekers are deterred from future visits when they realize emergency physicians 'can communicate with other hospitals, they will go to their primary care doctor.'

I think [the HIE] helps you a lot to figure out who are genuine patients, who are not genuine, who are looking for drugs, and who have been to different EDs and what has been done. So I think it would be of benefit for all the doctors.

\section{Conundrum of Protocols}

When discussing duplicative ordering, participants argued that internal protocols within individual EDs are a conundrum with both beneficial and problematic outcomes. The benefits consisted of expediting patients through the ED, which allows physicians to see more patients and also beneficial for patients by reducing their waiting time in the ED. However, participants also viewed protocols as problematic due to unnecessary ordering of diagnostics. 
The benefits of protocols was disputed as being more of a public relations initiative to reduce patient wait times in the ED, especially since the inception of hospitals posting wait times on the internet for the public. One participant argued that the problem with protocols is the increased ordering of unnecessary tests, which is not in the best interests of quality of care and escalates costs.

I don't get to order the tests on the patients. They are already ordered for me. And you don't want to get me talking about that because I think we are ordering far too many tests on too many patients all the time. But if I had control over this environment I would order a lot fewer tests and it would have nothing to do with me seeing the tests that have already been ordered someplace else. I would order them because I think the patients don't need them in the first place. They're on protocols. It is just because the premium is being placed by administrative individuals who think that speed is of paramount importance. And that it is the premium ... they're wanting to be so market competitive and so customer service savvy that they feel that ordering, getting things done quickly is the best thing that might happen for a patient when they come here. And in some ways, it is, but in other ways, it can be dangerous, it's very wasteful, and it's unnecessary, but we're not doing things scientifically all of the time now.

\section{Fiscal Benefits}

The amount of resources saved as a result of using the HIE varied when participants attempted to estimate the reduction in volume of ordered diagnostics, transmitted records via fax or non-electronic methods, or number of medical records reviewed. On the negative side, participants reported decreased productivity with internal electronic documentation, which necessitated the hiring of scribes to help the emergency physicians recoup some of the lost productivity. Two of the four study sites employed scribes in the ED. In addition, participants frequently identified the laborious practice of printing HIE information as a common practice, which adds costs to the department.

Reducing diagnostics. Participants estimated that the volume of duplicative ordering of diagnostics was reduced from 'several a month' to maybe 'one or two per month.' CT scans were the most frequently cited diagnostic; however, most participants acknowledged the recent publicity surrounding the amounts of radiation patients are receiving and the associated number of CTs, which may have influenced the focus on CTs. Participants did not estimate the cost savings and some participants stated the HIE did not reduce the number of CTs ordered.

[The HIE] hasn't decreased my CTs at all. Because my lawsuits are still coming in. So, I don't care about you know, I'm ordering them. 
Obtaining medical records. Obtaining medical records is inefficient, laborious, and often impossible or very slow to obtain after hours when medical record offices are closed. Participants reported that the HIE was efficient in both time and labor when compared with obtaining paper medical records.

Reviewing records. Participants claimed that knowing the correct primary care physician is important when admitting patients, arranging referrals, or trying to provide seamless delivery of care. Because patients do not know or cannot remember their primary care provider's name when in the ED, participants stated they have to admit the patient to the on-call physician. Admitting a patient to the wrong physician creates problems, including less than seamless delivery of care for the patient, but also wasted time resolving the confusion and ensuing conflict between hospitalists, emergency physicians, and the patient's primary care physician. Participants proposed that if the HIE consistently contained the name of the patient's primary provider, the problem 'would be alleviated.'

Before the HIE, hours [were] spent in medical records room looking through charts and finding information.

Lost productivity with EHRs and HIEs. Participants reported that using the EHR and accessing electronic documentation in the HIE reduced the productivity of the emergency physician. One participant stated that productivity of an emergency physician was reduced by $30 \%$. Even with the addition of scribes, participants contended that productivity is reduced when using electronic documentation compared with handwriting physician notes. Participants also stated that because the HIE was not user-friendly or intuitive to navigate, retrieving information 'slows them down.' Other problems were encountered when emergency physicians tried to find information in the HIE from unfamiliar EHR systems.

[The HIE] is slow, it is a slow process... I would love it if it were faster and easier to use.

In general, if [the EHR] is implemented really well it seems to be in about a year's time you get back to the level of productivity you used to have. If it is implemented poorly then you never get that back.

\section{Theme 5: Current View}

Participants verbalized that many changes were needed if the HIE was to be successful and sustainable in the long-term. The most cited change was the HIE system needed to be more user friendly and less cumbersome to use. Other frequently cited changes were the addition of emergency physician notes, including scanned copies of handwritten notes from non-electronic documenting emergencies, the addition of both 
EKG tracings and radiographic images to the HIE, and pharmacy integration to provide medication histories. Some participants stated they had never seen a physician note in the HIE, while other participants stated the physician note would not change their decisions or stated that 'it's usually not very helpful.' Other participants argued that the emergency physician note was very beneficial. Not all participants verbalized a need for the physician note, but participants were unanimous that EKG tracings in the HIE would be a valuable addition.

For sites using electronic documentation, participants proposed integrating the medication administration record containing the patient's medication history in the EHR with pharmacies. Incomplete medication histories were frequently mentioned as an ongoing problem in EDs. One study site used additional resources by hiring pharmacists in the ED as a solution to handling patient medication histories. When pharmacists were not available, or in the study sites not employing pharmacists in the ED, nursing resources were used to identify medications that was 'pain-staking and time consuming.' The participants also reported that because they lacked a complete medication history the patient may inadvertently be sent home on duplicative medications.

The question of how much information should be in the HIE resulted in divergent responses. Some participants wanted all the patient information that was available placed in the HIE while other participants wanted a minimal amount. Some participants proposed a separate ED module that would only contain ED pertinent information, which accommodates a user-friendly approach for emergency physicians accessing information. These participants stated that excess information in the HIE beyond what was needed for emergency medicine would only slow accessing and retrieving information and discourage use. Other participants viewed separate modules as an additional expense and unnecessary.

Participants in EDs with an electronic documentation system proposed integration of the EHR with the HIE, which would be more user-friendly and provide easier access to the HIE. Participants stated this might increase use of the HIE. Other changes proposed by participants were collated into the following categories:

1. Correct design flaws.

2. Standardize information contained in the HIE.

3. Provide HIE access to physicians at the same time physician is credentialed.

4. Integrate pharmacies and state pharmacy systems.

5. Provide functional portable devices.

6. Ensure speed in accessing information.

7. Provide education and regular hands-on training sessions for emergency physicians.

8. Design HIE and EHR systems to alert emergency physicians if patients have an HIE record.

9. Expand the HIE to include all hospitals in the city and extend beyond to the patient catchment area, including clinics, ambulatory centers, physician offices, psychiatric centers, and radiology clinics. 
10. Seek emergency physician feedback and input into the function and design of the HIE.

When participants were asked if they wanted the HIE removed, all participants verbalized that they did not want to see the HIE removed from their daily clinical practice. The responses varied from 'overall, the HIE was doing a pretty good job of getting everything the emergency physician needed to know' to 'there are a lot of gaps and the system needs a lot of tightening.' However, other participants also expressed that although the HIE had 'drawbacks, the system was in the best interest of patients.' Some participants viewed beyond the EHR to a personal health record and a national HIE system.

I think they can continue to try to make [the HIE] so if you go anywhere within the United States ... if I get sick in California, somebody can put my social security number in and know exactly my allergies, what medications I am on, and my past medial history. That helps the physician, I think it helps us treat the patient. It helps when I know, especially if you're in a car wreck and you can't speak for yourself ... I think that would be great if we could do that for every person in the United States. Every hospital, any hospital I went to.

Generally, participants expressed that the HIE had more positives than negatives although they frequently expressed considerable frustration with the less than optimal accessibility. They all verbalized that the HIE must be more user-friendly for the emergency physicians to increase use of the system. Some participants were reserved in their response when asked if they would miss the HIE.

It is just not as easy for me as it should be.

I probably would [miss the HIE], I don't know how much. It probably would hamper it just a little bit but I don't know how much it would hamper it but it probably would.

One participant viewed the HIE as a success and a great model. Another participant verbalized that he had concerns that patients would feel their confidentially was betrayed if they fully understood that information was being transmitted among facilities, but he also reported that other patients 'are actually pretty happy when you can pull [information] up.'

\section{Theme 6: Non-Use or Reduced-Use of the HIE}

The reasons participants reported why emergency physicians either do not want to non-use the HIE or have reduced usage rates were collated into nine categories. The most cited reason was the HIE is not user-friendly and impedes the workflow of the emergency physician (Table 4-3). The categories for reconciling non-use of the HIE are: 
Table 4-3. Participant Reasons Why Emergency Physicians Do Not Use the HIE or Have Reduced Usage Rates

\begin{tabular}{|c|c|c|c|c|}
\hline Participant & Reason \#1 & Reason \#2 & Reason \#3 & Reason \#4 \\
\hline 1 & Bias $^{\mathrm{a}}$ & Champion $^{\mathrm{b}}$ & Medical legal $^{\mathrm{c}}$ & $-{ }^{k}{ }^{k}$ \\
\hline 2 & User-friendly $^{\mathrm{d}}$ & Culture change $\mathrm{e}^{\mathrm{e}}$ & ---- & ---- \\
\hline 3 & User-friendly & ---- & ---- & ---- \\
\hline 4 & Competition $^{\mathrm{f}}$ & Education $^{\mathrm{g}}$ & User- friendly & Champion \\
\hline 5 & Revenue $^{\mathrm{h}}$ & Time $^{\mathrm{i}}$ & Medical legal & ---- \\
\hline 6 & ---- & ---- & ---- & ---- \\
\hline 7 & Education & Competition & ---- & ---- \\
\hline 8 & User-friendly & Education & Culture change & Medical legal \\
\hline 9 & Medical legal & User-friendly & Time & Culture change \\
\hline 10 & No privileges ${ }^{\mathrm{j}}$ & Medical legal & ---- & ---- \\
\hline 11 & User-friendly & User-friendly & Medical legal & ---- \\
\hline 12 & User-friendly & Time & Bias & Revenue \\
\hline 13 & User-friendly & Culture change & ---- & ---- \\
\hline 14 & No privileges & User-friendly & ---- & ---- \\
\hline 15 & Time & ---- & ---- & ---- \\
\hline
\end{tabular}

a Bias: Participants do not want to bias their own medical opinion.

${ }^{\mathrm{b}}$ Champion: Lack of a HIE champion.

${ }^{c}$ Medical legal: Threat of lawsuits.

${ }^{\mathrm{d}}$ User-friendly: Difficulty accessing HIE.

${ }^{\mathrm{e}}$ Culture change: Change in practice to using the HIE.

${ }^{\mathrm{f}}$ Competition: Competition between facilities for patients.

${ }^{g}$ Education: Lack of HIE education or hands-on training.

${ }^{\mathrm{h}}$ Revenue: No incentive for providers to decrease ordering diagnostics.

${ }^{i}$ Time: EDs are too busy; not enough time to access HIE.

${ }^{j}$ No privileges: Failure to grant emergency physicians access privileges to HIE.

k ----: Participant did not provide a response. 
1. Failing to provide a user-friendly HIE coupled with time constraints.

2. Medical legal concerns.

3. Biasing clinical decisions.

4. Lacking HIE education.

5. Changing the culture.

6. Competition.

7. Lost revenue.

8. Lacking HIE champions.

9. Failure to grant access privileges.

\section{Failing to Provide a User-Friendly HIE Coupled with Time Constraints}

Participants reported that difficulty in accessing the HIE is accountable for marginal or no use of the HIE system. The HIE should be 'easily accessible' with a minimal number of computer 'clicks' to gain access. The more clicks needed, the less likely the emergency physician will take time to access the information. EDs are busy and any 'extra steps' are considered 'hard to do.' Excess time spent accessing the HIE is 'laborious' and the participants viewed the HIE as a tool that is not user-friendly. Navigation within the HIE is 'cumbersome' and not 'intuitive.' Participants expressed frustration if information is missing, such as late data entries, because time spent to access the HIE is wasted time; time that could have been spent seeing more patients. Participants using electronic documentation in the emergency contended the HIE and EMR should be 'integrated' to facilitate ease of use and eliminate extra steps. Even one extra click during a busy emergency shift was voiced as being 'painful.' Participants stated that, 'the easier the access, the more I would use the HIE.'

\section{Medical Legal Concerns}

Medical malpractice suits are a reality for emergency physicians and a 'terrible problem' that impacts the way physicians practice medicine, which participants described as 'defensive medicine.' Although there are 'blatant problems' such as wrong site surgery, participants expressed a need for solutions, such as how some states have instituted caps and malpractice rates have decreased, or tort reform. Other participants contended that even with the medical legal ramification, they 'can document what they see and what they have' from the HIE; however, each case is dependent on the scenario. Other participants indicated that some emergency physicians would not rely on the results from other facilities because of the threat of lawsuits in the event they 'missed something.' Participants stated that they must do what is necessary to 'legally protect myself.'

I have asked doctors, why are you repeating these tests? This patient just had this test a few days ago. Well, I'm responsible now. The patient comes to me. What if I miss something. What if they missed something over there. So that is the what if. 
Participants also stated that in addition to the medical legal concerns, emergency physicians must report suspected drug diversions and accessing the HIE provides an audit trail. The reporting is time consuming and with so many patients visiting the emergencies and seeking drugs, emergency physicians avoid using the HIE. Other participants identified that they accessed the State prescription monitoring database and not the HIE.

With it being [State] law right now that if you suspect drug diversion you have to report it. That if you look up on the HIE and you see a patient had 12 visits for tooth pain ... you kind of now have lost the plausible deniability that you didn't think they may be diverting drugs or drug seeking. I believe the HIE is tracked when you log in so there is a paper trail there, so if you do that and see that, and don't report it you could be criminally liable. I don't know how much that's an actual thought about why we are using it versus the theoretical, I think that mostly it's an extra step.

I mean to me [the HIE] is much akin to the State ... prescription-monitoring database. That's been one of the greatest things that they've done in ages. Where I can see what narcotics have been prescribed to somebody and I use that multiple times a day on patients that have chronic pain issues or requesting medication refills for narcotics and stuff.

\section{Biasing Clinical Decisions}

Participants verbalized that emergency physicians want to form their own clinical decisions and not 'bias' their opinions by accessing the HIE. The physician will do 'what they need to do' and may or may not take the time to access the HIE. Other participants argued they will not bias themselves by reviewing the HIE prior to seeing the patient because that is not how they were taught to practice medicine. They want to form their own opinions.

\section{Lacking HIE Education}

Participants identified the lack of education and hands-on training as a reason emergency physician are not using the HIE. Because the HIE is 'cumbersome' to use and difficult to 'navigate,' some participants indicated that without someone to show them how to use the functions and speed up their access, they just do not have the time to use the HIE or learn the functions on their own. Some participants stated that they have 'heard almost nothing about the HIE' and without educational sessions there is no opportunity to learn more about the system and how the HIE can benefit them in clinical practice. 


\section{Changing the Culture}

Participants stated that using the HIE in daily clinical practice is a culture change. Emergency physicians were practicing emergency medicine before the implementation of the HIE and in their opinion they were 'doing fine,' or they 'think they do not need' the HIE. Other participants identified that the extra steps to access the HIE would not change their clinical decisions and that habit influences how physicians practice. Habits take a 'long time to break' and as a result, participants stated there are emergency physicians that have never used the HIE.

I know one [emergency physician] in particular that doesn't use [the HIE] at all, he doesn't even have access to it. He doesn't want to have access to it because he looks at it as another step and delay in getting information that is not really going to change what he does anyway.

\section{Competition}

Participants identified that competition between facilities and the importance of patient loyalty to a facility are factors that influence the use of the HIE. Hospital systems are businesses and are not always willing to share patient information that might result in patients using the services of other facilities. The transmission of patient information to all the facilities in the HIE database is open to the scrutiny of other professionals with some participants proposing there is the additional 'fear of malpractice.'

Hospital systems, especially hospital systems, who are competitors still like to keep their own. There is one of the barriers. I know this sounds crazy, but one of the barriers is that there is a feeling that if another physician or another health care provider looks at what I did, he may not like it, and then you open a door. That open door is, well he didn't do it right. Does that open up a malpractice issue or standard of care issue? So there's always that fear. I think that a lot of it is still based on a fear of malpractice.

\section{Lost Revenue}

In this economy, participants stated that revenue sources to the hospital and to the ED are factors that influence how the HIE is used. In areas with more insured patients, there is no incentive for providers not to order the maximum amount of diagnostics as a revenue generator. Patients with insurance will receive more diagnostics because of the billing potential and revenue; whereas, with the uninsured there is no benefit to ordering more tests. Providers in high insurance areas do not want to access the HIE since they will not use the information in the HIE to avoid ordering diagnostics. 


\section{Lacking HIE Champions}

Participants viewed that having an HIE champion in the ED may influence other emergency physicians to use the HIE and increase usage rates. The facilities are not communicating and therefore the successes one department experiences and how they reduced barriers, such as 'decreasing the number of clicks' to access the HIE, is not being shared between emergencies. Participants identified that champions are necessary in the departments to assist emergency physicians and increase usage, but only one participant identified himself as a champion of the HIE. This participant was not using electronic documentation in the ED.

\section{Failure to Grant Access Privileges}

One of the greatest deterrents to non-use of the HIE is failing to grant access privileges to emergency physicians. Two participants did not have access and participants identified other physicians within the departments who did not have access for a variety of reasons. Some physicians did not want access while others found the process too complicated and time consuming. The participants also reported that some emergency physicians were 'not aware' of the HIE in the departments. Participants without access relied on nurses to extract HIE information with one participant reporting he 'looked over the nurse's shoulder' to see HIE information, while other facilities did not grant access to nurses. Increasing the HIE usage is dependent on every emergency physician having access. 


\section{CHAPTER 5. DISCUSSION}

\section{Introduction}

This chapter discusses the study findings along with related literature, and truthfulness and credibility. A plausible interpretation is presented of an analytic explanation of the phenomena health information exchange (HIE) usability among emergency physicians. The chapter concludes with limitations, and implications and recommendations for practice and further research. This grounded theory qualitative study generated a substantive theory that explored and explained how and why emergency physicians use HIEs from the point of view of emergency physicians.

Following is a discussion of the results with details that support the conceptindicator model (Charmaz, 2006), which is a method of theory construction whereby the researcher uses the concepts grounded in empirical data to explain the relationships of the emergency physician participants in the phenomena of HIE usability. This study generated a substantive theory.

\section{Summary of Study}

The purpose of this qualitative study was to understand the perspectives of emergency physicians concerning the usability of HIEs. Usability is the effective, efficient, and satisfied use of the HIE with which emergency physicians can achieve delivery of medical care specific to emergency medicine in emergency departments. The fundamental question was how do emergency physicians use the HIE in making clinical decisions? The researcher's intent was a better understanding of how emergency physicians decided to use the HIE in making clinical decisions. This understanding would provide insights about how to achieve HIE usability for emergency physicians. Satisfied end-users who view the HIE as effective and efficient should use the HIE more.

However, this requires removing challenges and barriers while recognizing more benefits to using the HIE, and addressing the underlying reasons for not using the HIE. Understanding the complexities of using the HIE and providing solutions to increase usability of the HIE may influence greater use of the HIE in clinical decisions with demonstrated positive outcomes for patients.

Rich and thick data were collected using theoretical sampling following the guidelines of grounded theory. Contextual factors were identified using the paradigm of conditions, interactions/emotions, and consequences that helped link categories (Corbin $\&$ Strauss, 2008). For instance, multiple barriers or challenges when using the HIE causes emergency physicians to become frustrated and dissatisfied when faced with overcrowded waiting rooms with the consequence that they abandon using the HIE. Segments of data were coded using first cycle (in-vivo, initial) and second cycle coding methods (focused, theoretical), along with analytic memos. Analyzing the data followed the process of generating, developing, and verifying concepts using constant comparative 
analysis. The aims of the analysis were progressive from description, conceptual ordering, and developing theory. Concepts from the coded segments were developed into categories, core categories, themes, and finally an overarching theoretical scheme visualized in a conceptual framework that generated a substantive theory.

A substantive theory was generated that using the HIE among emergency physicians is the process of rationalizing non-use and reconciling challenges and benefits (Figure 5-1). The antecedent condition of usability was identified from the participants during the interviews as a typical day in the ED and why the participants accessed the HIE and under what conditions. Six major themes emerged from the data analysis of this study:

1. All the participants indicated they used the HIE; however, the characteristics of the HIE user, frequency of use, experience using HIE systems, and who had privileges to access the HIE were variable.

2. The majority of participants reported that the HIE influenced clinical decisions, but there are many factors, including information missing in the HIE or the inability to track, compare, and trend data results. Although accessing the HIE was a factor in the reaction time needed to intervene in a patient episode, other factors such as duplicative testing was characterized along with the concept that the HIE can be life-saving.

3. All the participants expressed struggling with the many challenges and barriers of using the HIE, but the core barrier to consistent and higher usage was the difficulty in accessing the HIE.

4. All the participants cited benefits of using the HIE but highlighted avoidance of duplicative testing, increasing efficiency in patient flow, and decreasing costs.

5. All the participants reported that changes are needed in the current HIE system in order for usage to increase.

6. The majority of participants expressed an opinion as to why some emergency physicians did not use or had low usage rates of the HIE. The reasons for not using or reduced-use of the HIE were collated into nine categories.

Theoretical sufficiency occurred when the properties, dimensions, and variations of each category were sufficiently developed for the purposes of this research and that further sampling was adding little to the conceptualization (Corbin \& Strauss, 2008). "In reality, a researcher could go on collecting data forever, adding new properties and dimensions to categories ... accept what has not been covered as one of the limitations of the study" (Corbin \& Strauss, 2008, p. 149). 
Using the HIE among Emergency Physicians Is the Process of Rationalizing Non-Use and Reconciling Challenges and Benefits

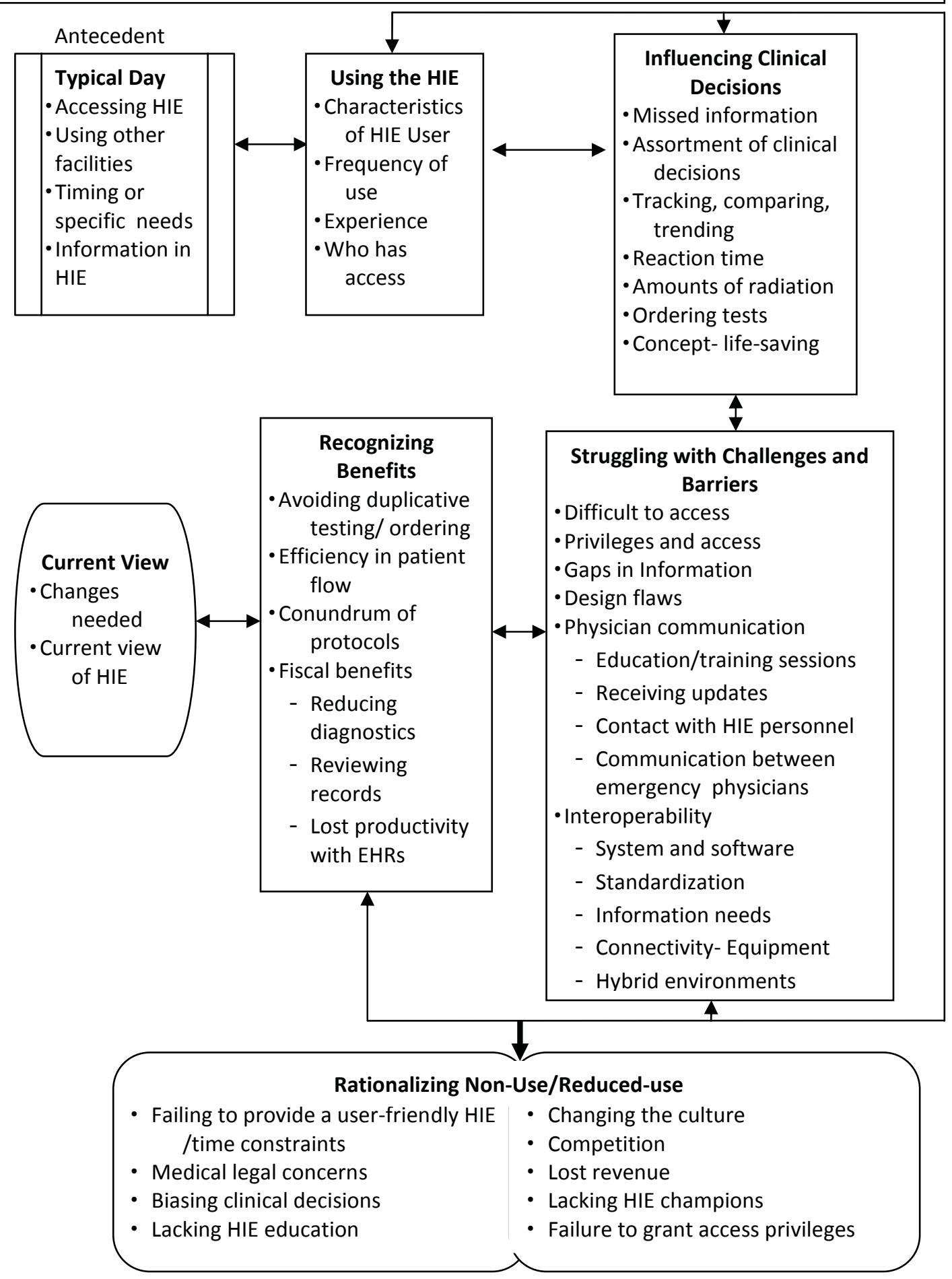

Figure 5-1. Substantive Theory: Process of Rationalizing Non-Use and Reconciling Challenges and Benefits 
The overriding finding in this study discovered that emergency physicians gave good reasons why the HIE is not being used for the majority of patients while these physicians also reconciled the challenges and benefits of using the HIE to explain the role of HIEs in making clinical decisions. There is a disconnect in the necessity of using the HIE to make clinical decisions and any negative outcomes that may occur in patients from not using the HIE. Generally, emergency physicians viewed the HIE as not being user-friendly and that they probably do not use the HIE as much as they could for making clinical decisions. The perspective of the emergency physicians was the ED environment is too busy and because the HIE is less than user-friendly as needed by physicians to practice emergency medicine, the HIE disrupts workflow and is a deterrent to consistent high usage in making clinical decisions.

\section{Truthfulness and Credibility}

Credibility indicates, "That findings are trustworthy and believable in that they reflect participants, researchers, and readers experience with a phenomenon but at the same time the explanation is only one of many possible plausible interpretations possible from the data" (Corbin \& Strauss, 2008, p. 302). Five strategies were used for increasing the truthfulness (Corbin \& Strauss, 2008; Silverman, 2006) of the findings:

1. Refutability principle: Assumptions were refuted, such as low rates of reported HIE usage among emergency physicians was the effect of the physicians not wanting to use the HIE because they saw no value in using the HIE in clinical decisions. However, using an inductive process during data analysis revealed that access was not sufficient and the process was not easy and thus a barrier to use.

2. Constant comparative method: Data segments were compared across interviews. This was accomplished by initially comparing coded segments, and as categories emerged, hypotheses were compared with the data corpus. For example, initial hypotheses questioned if HIE usage was different for physicians using the EHR to document versus physicians handwriting documentation.

3. Comprehensive data treatment: All the data were incorporated from the 15 interviews into the analysis, which generated 1135 coded segments and 192 memos. Early analysis involved intensive coding that progressed to delineating categories. In addition, simple counts were used to highlight usage rates and the descriptors of high, medium, and low use reported by the participants.

4. Searching for deviant cases or negative cases that do not fit the pattern: For example, participants were using the HIE and did not have access privileges.

5. Transcribing text: The face-to-face interviews were audio recorded, transcribed verbatim, and reviewed for accuracy by the researcher, followed by grammatical corrections and removal of utterances. Although the researcher did not transcribe to the level of conversational analysis, excessive pausing and hesitation during the 
responses were noted in memos. Brackets were used to clarify the meaning, such as participants referring to the HIE as it, and latching was used to indicate that there were no pauses between the researcher speaking and the participant speaking, which denoted that the researcher was unable to finish the question before the participant responded.

In presenting the findings, low-inference descriptors, or verbatim accounts from the participants, are used extensively to demonstrate the credibility of the researcher's interpretations. Appendices are included of interview protocol as approved by the Institutional Review Boards (Appendix $\mathbf{A}$ and $\mathbf{B}$ ) and in-depth descriptions of conceptual definitions used in the data sets (Appendix C). Additional verbatim accounts are provided to support the findings (Appendix D). During the interviews, the researcher paraphrased the participants' responses as a check for accuracy of what the researcher was interpreting from the participants' recounting of the phenomena. In addition, the methodology and findings were reviewed by committee chair, a qualitative researcher, for logical flow, depth, variation, and applicability of findings.

\section{Discussion}

This chapter, which is aligned with the antecedent condition and each of the six study themes, provides this researcher's interpretative insights into the results. The analysis of the phenomena is reconstructed into a plausible interpretation about the usability of the HIE as experienced by emergency physicians.

\section{An Analytic Explanation: HIE Usability as Experienced by Emergency Physicians}

Using the HIE is a repetitive process of emergency physicians giving good reasons for not using the HIE during an emergency shift while reconciling the many challenges with the benefits of accessing the HIE. For emergency physicians, a typical day in the ED is busy with patients frequently moving between different EDs. Emergency physicians are challenged to handle the volume of patients while moving patients quickly through the ED. The recent implementation of providing the public with wait times through the internet only encourages the public to view the ED as a non-urgent clinic rather than a specialty hospital unit for true emergencies. The emergency physician has to manage time between the urgent or true emergency patient and an overcrowded waiting room of non-urgent patients. "In Indianapolis alone, approximately 50\% of the population sought care in at least one of the EDs over the four year study ... a substantial fraction visit a separate healthcare system" (Finnell et al., 2005, p. 248).

Using the HIE is not a priority because emergency physicians are there to practice emergency medicine and that means accessing the HIE on a selected basis. There is no good time to access the HIE because there are no set criteria for when to use the HIE, whether before or after examining a patient or for a specific need such as suspicions about motives (patients seeking drugs). Emergency physicians are not primary care 
providers; however, patients visit the ED for multiple reasons other than true emergencies and expect emergency physicians to provide interventions like a primary care provider. While some patients are poor historians, other patients visit the ED rather than waiting for their next appointment with their primary care provider. The emergency physician has to piece together a medical picture for patients they are seeing for the first time and may not see again. Other patients are frequent flyers who rely on EDs for their primary source of medical care and therefore lack the seamless provision of care provided through a primary care provider. The HIE has become one more tool for emergency physicians to try avoid duplicative testing and duplicative medication prescriptions, or trying to find medical information about the patient from other facilities to piece together some sort of medical history for patients who should be seeing their primary care provider. Although the HIE is thought to produce positive outcomes, evidence is lacking to provide scientific evidence for emergency physicians, but rather the focus is on the economics of saving health care dollars. "Health information exchange could make emergency care less expensive, more efficient, and safer for patients ... several existing models predict that many of these benefits [such as quality, safety, workflow efficiency] will be realized; however, only minimal data have been generated thus far, and much more remains to be done" (Shapiro et al., 2006, p. 430). Others argue that the costs of maintaining a nationwide HIE for all people over the course of their lives will produce staggering costs. Financial implications are a major concern for all stakeholders when determining HIE adoption (Kaushal et al., 2005). Significant resource allocation is required by organizations for initial HIE implementation along with major change management strategies (Doebbeling, Chou, \& Tierney, 2006) while additional pressure is exerted on internal IT departments (Frisse, M. E., 2005).

Emergency physicians used the HIE even though it is not an easy process. They expect to find patient information if the HIE shows a visit history, but they are never quite sure what will be available because no one has informed them of the restrictions or specifics of available information, such as why some visit histories have ED physician notes and others do not. It is frustrating to take time out to access the HIE only to find gaps in the information. Minimal disruptions to workflow are essential to prevent long wait times, which are viewed as an inefficient ED. "Even a small delay in retrieving healthcare information makes that information essentially useless for emergency care ... if providers cannot retrieve information quickly, it won't be used at all" (Finnell et al., 2005, p. 246).

The process of using the HIE is further complicated because some emergency physicians have not been granted access privileges and have to find alternate means to get information from the HIE, all of which are inefficient and place further burdens on a busy ED. However, the problems are compounded by general confusion of not knowing who in or outside the ED had access. Some facilities grant privileges to nurses and consultants, while other facilities deny HIE access to nurses and consultants but gave access to scribes who are hired in the ED to document for the emergency physicians. The amount of time an emergency physician has been using the HIE or whether they documented electronically or manually does not influence HIE use. Some emergency physicians routinely work in EDs who are not in the HIE system and maybe this 
contributed to their not relying on the HIE to make clinical decisions or being indifferent when asked if they would miss the HIE; however, other emergency physicians who consistently have access to the HIE also stated that they only occasionally make clinical decisions based on information found in the HIE. "The presence of a certified EHR was not associated with adoption or implementation - a plausible scenario as HIE can and does exist with an EHR ... HIE is not a naturally occurring phenomenon when an EHR is present" (Vest, 2010, p. 801).

Emergency physicians have variable views as to what constituted high or low usage of the HIE. Is using the HIE for $5 \%$ of the patients seen during a shift high usage or is $38 \%$ or $77 \%$ ? Even though some emergency physicians may view single digit percentages of use as being high, overall the HIE was seen as a tool that provides better care and a better understanding for an improved quality of life for patients. Emergency physicians want to know what is going on with patients and what has already been ordered by taking the guesswork out of the patients' recounted medical history. Deferring further interventions by using the information in the HIE is beneficial for the patient, but also the emergency physician who can see more ED patients. Nevertheless, it appears that the majority of clinical decisions are not dependent on using the HIE because the overall self-described usage rates indicated that the HIE is accessed for less than half of the ED patients examined in a shift while other physicians admitted to never using the HIE during a shift. When identifying the need for formal evaluations of the HIE, Frisse (2010) reported early results following a HIE implementation that showed when $13 \%$ of individuals presented at one emergency department with data available from other sites from the prior month, the HIE was accessed for only $26 \%$ of the ED visits.

How the HIE influences clinical decisions is variable even though physicians agree that the more information available the better the decision-making. Patients do not always give accurate or complete information for a variety of reasons and the HIE helps the emergency physician pick-up information and piece together a clinical picture. Having even a partial history of patients' allergies, previous work-ups, or medications influences clinical decisions and at times was described as being life-saving. The information might not change what the emergency physician was going to order in a specific patient encounter, but the information helps alleviate confusion such as when a patient revealed they just had a CT scan two days ago at another ED but cannot recount the findings. The emergency physician may still order another CT but the clinical picture is clearer for an emergency physician who may not have the opportunity to re-evaluate the patient once they leave the ED. In a systematic review of literature, Fontaine (2010) stated, "The evidence that HIE improves the quality and safety of care is more limited, yet it is certainly reasonable for practices to conclude that speedier access to clinically useful information will benefit patient care and prevent errors" (p. 666).

Emergency physicians need tools such as the HIE at their disposal to make critical decisions of when to order diagnostics and when further testing could be deferred. But the reduction of diagnostics is influenced by physician practice behavior, which varied from working patients from scratch, as described by one participant, to only focusing on the immediate complaint. The issues become more complex when physicians question if 
a radiology report suffices for an image or the reality that laboratory values can change in short time frames and the emergency physician may not see the patient again to reevaluate. Shapiro et al. (2007) found in a questionnaire of emergency physicians that EKGs and X-ray images, and discharge summaries were ranked as the highest data preferred elements.

Patients demand care in EDs and as a result, the amount of radiation patients receive is being discussed. How much radiation is too much when patients are using multiple facilities? "EDs today provide much of the medical care for patients without medical insurance" (Institute of Medicine, 2006, p. 115). Although using the HIE to track and compare test results may alter a patient's disposition and is viewed as saving time and money for the overall health system, the needs to make the HIE easier to use becomes critical when pertinent information is missing, HIE functions are poorly understood, or as one emergency physician stated when reviewing EHR information, ' $20 \%$ is valuable information and the rest is just junk.' How the HIE influences a patient's clinical course in the ED has many questions. "The potential for HIE to reduce costs and improve the quality of health care in ambulatory primary care practices is well recognized but needs further empiric substantiation" (Fontaine et al., 2010, p. 655).

Emergency physicians identified multiple challenges and barriers when using the HIE, from the lack of standardization within the HIE to problems with software communication, that either restricted or deterred increased use. They want to have a usable system but for many emergency physicians the HIE is seen as both cumbersome and not intuitive. Easy and quick access is imperative if the HIE is to blend into the workflow of a busy emergency physician who has not had on-hands training or education sessions on the use and functions of the HIE. The majority of physicians do not see any efforts to make the HIE system more user-friendly, which is both discouraging and frustrating and maybe the cause of many physicians simply not showing much interest in the HIE. "It is of the utmost importance that the user interface be well designed and that clinicians be involved in the planning and implementation process" (Shapiro et al., 2007, p. 704).

The HIE is not integrated with the EHR, which further disrupts workflow. Too many clicks is viewed as a major obstacle and cited in the literature as a cause for frustration and resistance to use. "When health information technology does not have this level of customization [data exchange and aggregation support within specific environments] invariably, some group of users will be less satisfied with the number of mouse-clicks required to perform a function they commonly perform" (Johnson \& Gadd, 2007, S22). Screens logging out too quickly require frequent and repetitive logins, or too much scrolling, multiple steps retrieving information, connectivity failures, and poorly designed computer stations are all flaws and annoyances that many emergency physicians claim could have been avoided if the emergency physicians themselves had been more involved in how the HIE functions in the ED.

Pharmacists are hired in some EDs to try sort out what medications patients are taking or nurses are phoning local pharmacies trying to get a medication history to avoid 
duplicative medications, which one emergency physician described as, "pain staking and time consuming.' Inefficiencies are frequently encountered such as nurses printing information from the HIE to give to physicians only to shred the paper after the patient leaves the ED. If the HIE is truly going to function as a usable tool for emergency physicians, they need to be involved and have a voice in the process of correcting current deficiencies. They want to be involved. "Barriers to achieving such efficiencies [reduced staff time, decreased support staff] include inappropriately redesigned workflow, lack of time to train staff, lack of technical proficiency among staff, and general lack of an IT culture" (Fontaine et al., 2010, p. 664).

Many emergency physicians view technology as being able to produce efficiency, but doubts arise when physicians work in the ED without access privileges, few hospitals have ED champions to answer questions or address challenges, and the HIE does not encompass the patient catchment area causing gaps in patient information. "There is a tremendous need for HIE in the EDs, and emergency physicians believe that having access to currently siloed data at outside institutions will benefit patient care and the efficiency with which health care is delivered" (Shapiro et al., 2007, p. 704). The emergency physicians are not familiar with how the HIE operates in other hospitals in the HIE system and the physicians themselves rarely discuss the HIE within their own departments. As one emergency physician stated, 'the HIE was rolled out and it was hereuse this,' which maybe gives emergency physicians the impression that the HIE is just not a priority in a busy ED when there are no efforts to listen to the emergency physicians and improve the system. "The sparse body of research on HIE efforts and anecdotal reports indicate the barriers to HIE adoption and implantation include factors beyond simply the presence or absence of a specific technology" (Vest, 2010, p. 797).

Despite all the flaws and challenges encountered on a daily basis with the HIE, emergency physicians see many benefits, even the physicians who seem not to be high users. One emergency physician saw himself as a strong advocate and champion for the $\mathrm{HIE}$ and was able to provide input and suggestions into the system. However, he also knew he was the exception and recognized that other EDs do not provide HIE education or have the same interest level in trying to make the HIE successful. Another emergency physician described the HIE as three pillars: improving patient care and safety, increasing efficiency, and realizing cost savings. Managing patients more efficiently decreases ED visits, moves patients through the ED faster, and decreases wait times. Avoiding duplicative testing, although the levels of avoidance ranged from substantial to minimal, was the most cited benefit. However, the emergency physicians quickly point out that other measures in the ED, such as automatic protocols, are nothing more than an administrative marketing strategy to be more competitive and give the illusion of customer service by decreasing patient wait times, but in reality automatic protocols increase unnecessary tests. "HIE may result in savings for the overall health care system by reducing redundant tests and through better medical decisions making that shortens hospital stays ... actual saving from HIE-enabled improvements of care have yet to be demonstrated" (Fontaine et al., 2010, p. 664). 
The emergency physicians see other areas of efficiencies and cost savings by no longer faxing large volumes of medical records, which is both labor intensive and slow. Using the HIE to indentify primary care providers saves reviewing medical records and prevents conflicts when a patient is admitted to the wrong physician. Regardless of technology benefits, emergency physicians also see other negatives such as the productivity of an emergency physician is estimated to drop by $30 \%$ with the implementation of electronic documentation. As result, some EDs are hiring scribes to electronically document for physicians.

Many changes are needed if the HIE is to be successful and sustainable in the long-term. "The failure rate of RHIOs [regional health information organizations] reflects the difficulty of HIE sustainability in open systems. Of RHIOs known to exist in July 2006, nearly one quarter were defunct by early 2007" (Fontaine et al., 2010, p. 656-7). Providing the information emergency physicians need and want, such as EKG tracings, standardized HIE content, pharmacy integration to provide medication histories, quick and easy access with speed in extracting information, and listening to emergency physicians are necessary steps to make the HIE more usable, that is effective, efficient, and satisfied use of the HIE with which emergency physicians can achieve delivery of medical care specific to emergency medicine in EDs.

Using the HIE takes commitment to overcome the barriers no matter how many benefits are apparent. Physicians want to do the very best for every patient in the ED regardless of emergent or non-urgent status. Although there might be more positives than negatives, the considerable frustration with less than optimal accessibility was more pronounced. HIE use is not just influenced by the act of weighing benefits against barriers, but also recognizing that there is an additional layer in the complexity of using HIEs, which is understanding the underlying reasons why some emergency physicians are reluctant to use the HIE or have lower usage rates. In a study to explore why old data are not accessed more often, audit logs indicated that users in the ED accessed clinical information from previous visits less than half the time even though they knew the information was available; the study concluded that there might not be enough time to review old data (Hripcsak et al., 2007), or as noted previously, the old data is not the type of data that is now needed.

The HIE must be user-friendly, which means it must be easily accessible with the minimal number of clicks. Fewer clicks mean more speed and less disruption to the workflow in a busy ED where more time translates into seeing more patients. While easy access is a major factor, there are other reasons, such as the reality of medical malpractice that forces defensive medicine or how physicians want to form their own medical opinion without the bias of having seen the patient diagnosis in the HIE. "Liability concerns center around providers not acting on external data made available through HIE, or for acting on external data that is inaccurate. There is scant legal precedent to offer guidance about the liability of a physician who acts on clinical information made available in such situations" (Fontaine et al., 2010, p. 665). The HIE is a change and changing the culture and practice habits take a long time to break and is complicated even more without education or champions available to the ED. 
Hospitals are businesses and are not always willing to share patient information that might result in losing patients to other competitive facilities. When discussing ambulatory primary care practices, Fontaine (2010) conducted a systematic literature review and found that competition creates conflict because HIEs share patients and their data, which competing parties consider their most valuable assets; this is further compounded by a fragmented and competitive United States health system. Decreased patient volume is less revenue. There is also a suggestion that insured patients receive more tests in some facilities that are more interested in generating revenue or transmitting medical records into a central database shared by many other entities opens the records to the scrutiny of other professionals and the fear of malpractice. "Insured patients increasingly turn to the ED during times when their physician is unavailable, such as evening and weekend, and they are often sent to the ED for tests and procedures that their physician can't easily perform in the office" (Institute of Medicine, 2006, 115).

In conclusion, HIE usability is a complex phenomena that encompassed many different challenges, barriers, and benefits that each individual emergency physician has to reconcile within themselves. The decision to use the HIE in making clinical decisions is often preceded by how busy the ED is at the time or how crowded the waiting room. Accessing the HIE is time consuming and when time is at a premium the HIE is likely not going to be used. But reconciling challenges with benefits, although a repetitious occurrence, is not the whole experience for emergency physicians who also have good reasons and can rationalize why they are only using the HIE sporadically.

\section{Strengths and Limitations}

The design of this study using grounded theory differs from previous literature related to HIEs and emergency physicians whereby studies did not use face-to face interviews and the development of a substantive theory. There are few qualitative or quantitative studies exploring the perspectives of emergency physicians related to HIE usability. At the time of this study, there is an absence of grounded theory methodology studies exploring the perspectives of emergency physicians specific to HIE usability.

There were multiple factors affecting HIE usability among emergency physicians in the specific sites for this study and a limitation of this study acknowledges that the factors may vary in a different geographical area and different RHIO. However, the factors identified in the literature review for physicians in general are similar to the factors identified by the participants in this study. The similarities strengthen the findings in the study; in addition, the findings in this study were more detailed and specific to the experiences of emergency physicians. The synthesis of the issues identified specifically by emergency physicians creates an in-depth understanding of the multiple issues directly related to emergency physicians and HIE usability.

Participants in this study were selected by the researcher using purposive sampling followed by theoretical sampling, but gatekeepers were identified prior to the collection of data to facilitate the researcher's access in the EDs. The gatekeepers could 
have influenced some of the results with the participants. However, the researcher carefully reviewed the study protocol with the gatekeepers to prevent any pressure being exerted on physicians to participate. The gatekeepers (Emergency Medical Directors) were an important component of the study since their involvement reduced the researcher being viewed as an outsider.

The researcher, who has both management and clinical emergency experience, concluded that prior emergency experience was a strength because of the rapport that was quickly established with the participants. In addition, being familiar with the workflow of busy EDs and the jargon was an asset during the interviews.

The majority of participants were male, which was representative of the gender distribution of the emergency physicians in all four study sites. Therefore, the small number of female participants is not considered a limitation.

Data collection occurred over an eight-week period. Although confidentiality was stressed with the participants, it was evident during the later stages of the interviews that emergency physicians were discussing the study among themselves in each ED. The interest in the study was considered a strength by raising awareness of the HIE and functions since the emergency physicians had identified the HIE was rarely if ever discussed among the physicians in the department. However, there was also concern not to bias potential participants. At this point, theoretical sufficiency had occurred and data collection was concluded. The researcher concluded that the biasing effects were minimal based on the responses and analysis of the data.

\section{Implications and Recommendations}

The results of this study can contribute to the existing knowledge of HIE usability and to the development of practice interventions and suggestions for further research. The following recommendations are based on the findings and conclusions of this study.

\section{Practice}

This study identified a number of challenges and barriers that emergency physicians face on a daily basis if they are going to use the HIE. By identifying the deficits in using the HIE system, from the perspective of emergency physicians, a unique opportunity is presented to begin correcting the problems and increase the physicians' satisfaction with the system. Usage should increase as emergency physicians become more satisfied with the HIE. RHIOs have been failing across the nation. As the emergency physicians use the HIE more in making clinical decisions, there is an opportunity for the RHIOs to demonstrate that the HIE is making a difference, which improves the sustainable and viability of the RHIO for this region. Providing empirical evidence of the effective and efficient use of the HIE, along with the expressed and measured satisfaction of emergency physicians using the HIE, will provide evidence of 
the usability of the HIE within the emergency physician community. Administrators and managers of RHIOs can use the findings as a starting point to address design flaws and interoperability issues identified as problem areas for the emergency physicians. For example, how can granting access privileges be made simpler to ensure that all emergency physicians have HIE access? Usability is the effective, efficient, and satisfied use of the HIE with which emergency physicians can achieve delivery of medical care specific to emergency medicine in emergency departments.

The emergency physicians identified the lack of educational sessions to provide information about the functions of the HIE. For example, graphing lab values was identified as an asset; however, few participants knew the graphing function was available. Other participants indicated graphing functioned only sporadically and they did not understand why the problem occurred or who to contact. Hospital administration is afforded an opportunity to address the issues with emergency physicians and provide ED champions to provide education as needed and to advocate HIE use. However, this is dependent on hospital administration having an interest in promoting the HIE as a useful tool in creating efficiency in the overall health care system by possibly reducing duplicative services. There is the risk of possibly losing patients to other EDs, but moving patients through the ED more efficiently improves patient satisfaction and enables emergency physicians to see more patients. As identified in previously published literature by Shapiro et al. (2006), HIEs could make emergency care less expensive, more efficient, and safer for patients. Again, revenue generation for individual hospitals will be balanced with providing cost savings to the overall health care system. There is a responsibility when incorporating new technology into the work environment that appropriate measures are provided to ensure that end-users are fully familiar with the functions.

State governments initiating and implementing statewide HIE networks will benefit from this study by having a concentrated research report amalgamating the issues of HIE usability from a specific physician group rather than using findings that collate physicians as one group. Identifying one group and the specific needs of emergency physicians has the potential to focus on improvements and demonstrate patient outcomes for one area (the ED) that can be expanded to HIE successes in other areas. The current infusion of money from both the state and federal levels, into health information technology, requires identification of specific areas to focus resources to provide the empirical evidence necessary to substantiate the value of HIEs in clinical decisions and patient outcomes. However, the underlying reasons identified by emergency physicians are also applicable to other health care providers and require policy intervention. For example, what policies are needed to provide standardized guidelines that remove the fear of medical malpractice liability for health care providers who use the HIE in clinical decisions?

Emergency physicians who are advocates for using the HIE in making clinical decisions, or emergency physicians just wanting to know about the HIE, can use the study findings to raise awareness of the issues identified as both problems impeding use and the underlying reasons deterring both emergency physicians reluctant to use the HIE 
and HIE late-adopters. The study findings provide emergency physicians the opportunity to understand the issues as expressed by other emergency physicians and might open the lines of communications between emergency physicians within their own departments and with emergency physicians in other EDs. Understanding the issues as expressed by the emergency physicians themselves provides a starting point to address the issues.

\section{Research}

The published literature identifies the need for further studies. Minimal empirical evidence is available that demonstrates HIE usability and patient outcomes. This study is unique in that it provides findings on HIE usability from the point of view of emergency physicians using the HIE and obtained through face-to-face interviews. This study was limited to four study sites in one geographic area, but the study could be extended to other EDs and geographic areas. The study could also be extended to other physician groups or health services areas. Insight into the concept of HIE usability among emergency physicians as described in this study could be compared to HIE usability among other physician groups and reveal how to improve the overall usability of the HIE. Further research on strategies to improve HIE usability could contribute to improved education and implementation interventions that affect improved sustainability of the HIE system.

Providing emergency physicians with empirical evidence is necessary to change practice habits. Suggesting the HIE has benefits is not the same as providing empirical evidence. While guarding the quality of care currently being delivered to emergency patients, further research is needed to identify the conditions and processes necessary to incorporate the HIE into making clinical decision in emergency departments. Further research is needed to establish that HIE usability is the effective, efficient, and satisfied use of the HIE with which emergency physicians can achieve delivery of medical care specific to emergency medicine in emergency departments.

Recommendations include studying HIE usability in different settings and with different health care providers to broaden the population and expanding the study to quantify the phenomena with hypothesis testing and identifying variables. Although generalization is not the purpose of qualitative research, the insight and understanding of the concept of how emergency physicians use the HIE can apply to many other health care providers in other settings (transferability). In this study, the analysis was moved from generalizations about a small sample of emergency physicians to the transferability of a substantive theory of an empirical problem, HIE usability. 


\section{LIST OF REFERENCES}

Adler-Milstein, J., Bates, D. W., \& Jha, A. K. (2009). U.S. regional health information organizations: Progress and challenges. Health Affairs, 28(2), 483-492.

Adler-Milstein, J., McAfee, A. P., Bates, D. W., \& Jha, A. K. (2008). The state of regional health information organizations: Current activities and financing. Health Affairs, 27(1), w60-9.

Armijo, D., McDonnell, C., \& Werner, K. (2009, October). Electronic health record usability: Evaluation and use case framework. Agency for Healthcare Research and Quality. Retrieved February 19, 2011, from http://www.ahrq.gov

Ash, J. S., \& Guappone, K. P. (2007). Qualitative evaluation of health information exchange efforts. Journal of Biomedical Informatics, 40(6, Supplement 1), S33-S39. doi:10.1016/j.jbi.2007.08.001

Baumlin, K. M., Shapiro, J. S., Weiner, C., Gottlieb, B., Chawla, N., \& Richardson, L. D. (2010). Clinical information system and process redesign improves emergency department efficiency. Joint Commission Journal on Quality \& Patient Safety, 36(4), 179-85 (24 ref).

Blumenthal, D. (2009). Stimulating the adoption of health information technology. New England Journal of Medicine, 360(15), 1477-1479.

Burns, N., \& Grove, S. K. (2009). The practice of nursing research: Appraisal, synthesis, and generation of evidence St. Louis, Missouri: Saunders Elsevier.

Charmaz, K. (2006). Constructing grounded theory: A practical guide through qualitative analysis. Thousand Oaks, CA: Sage.

Conn, J. (2011). IT: Set for a growth surge. Modern Healthcare, 41(1), 28-29.

Corbin, J., \& Strauss, N. (2008). Basics of qualitative research $3 e$. Thousand Oaks, CA: Sage.

Doebbeling, B. N., Chou, A. F., \& Tierney, W. M. (2006). Priorities and strategies for the implementation of integrated informatics and communications technology to improve evidence-based practice. Journal of General Internal Medicine, 21(S2), S50-S57. doi:10.1111/j.1525-1497.2006.00363.x

Englebardt, S. P., \& Nelson, R. (2002). Health care informatics: An interdisciplinary approach. St Louis, Missouri: Mosby. 
Finnell, J. T., Overhage, J. M., \& McDonald, C. J. (2005). In support of emergency department health information technology. AMIA-Annual Symposium Proceedings/ AMIA Symposium, 246-250.

Fontaine, P., Ross, S. E., Zink, T., \& Schilling, L. M. (2010). Systematic review of health information exchange in primary care practices. Journal American Board Family Medicine, 23(5), 655-670.

Frisse, M. E. (2005). State and community-based efforts to foster interoperability. Health Affairs, 24(5), 1190-1196. doi:10.1377/hlthaff.24.5.1190

Frisse, M. E., \& Holmes, R. L. (2007). Estimated financial savings associated with health information exchange and ambulatory care referral. Journal of Biomedical Informatics, 40(40), S27-S32.

Frisse, M. E. (2010). Health information exchange in Memphis: Impact on the physicianpatient relationship. Journal of Law, Medicine \& Ethics, 38(1), 50-7 (23 ref). doi:10.1111/j.1748-720X.2010.00465.x

Grossman, J. M., Bodenheimer, T. S., \& McKenzie, K. (2006). Hospital-physician portals: The role of competition in driving clinical data exchange: Hospitals are giving their physicians electronic access to patient data, but market competition impedes communitywide data exchange. Health Affairs, 25(6), 1629-1636.

HIMSS EHR Usability Task Force. (2009, June). Defining and testing EMR usability: Principles and proposed methods of EMR usability evaluation and rating Healthcare Information and Management Systems Society. Retrieved February 19, 2011, from www.himss.org/content/files/HIMSS_DefiningandTestingEMRUsability.pdf

Hripcsak, G., Sengupta, S., Wilcox, A., \& Green, R. A. (2007). Emergency department access to a longitudinal medical record. Journal of the American Medical Informatics Association, 14(2), 235-238.

Hripcsak, G., Soulakis, N. D., Li, L., Morrison, F. P., Lai, A. M., Friedman, C., Calman, N. S., \& Mostashari, F. (2009). Syndromic surveillance using ambulatory electronic health records. Journal of the American Medical Informatics Association, 16(3), 354361 .

Institute of Medicine. (2006). The future of emergency care in the united states health system. Annals of Emergency Medicine, 48(2), 115-120.

Johnson, K. B., \& Gadd, C. (2007). Playing smallball: Approaches to evaluating pilot health information exchange systems. Journal of Biomedical Informatics, 40, S21S26. 
Kaelber, D. C., \& Bates, D. W. (2007). Health information exchange and patient safety. Journal of Biomedical Informatics, 40, S40-S45.

Kaushal, R., Blumenthal, D., Poon, E. G., Jha, A. K., Franz, C., Middleton, B., Glaser, J., Kuperman, G., \& Christino, M. (2005). The costs of a national health information network. Annals of Internal Medicine, 143, 165-173.

Kern, L. M., Barron, Y., Abramson, E. L., Patel, V., \& Kaushal, R. (2009). HEAL NY: Promoting interoperable health information technology in New York state. Health Affairs, 28(2), 493-504.

Kern, L. M., \& Kaushal, R. (2007). Health information technology and health information exchange in New York state: New initiatives in implementation and evaluation. Journal of Biomedical Informatics, 40(6), S17-S20.

King, N., \& Horrocks, C. (2010). Interviews in qualitative research. Thousand Oaks, CA: Sage.

Kushniruk, A. W., Triola, M. M., Borycki, E. M., Stein, B., \& Kannry, J. L. (2005). Technology induced error and usability: The relationship between usability problems and prescription errors when using a handheld application. International Journal of Medical Informatics, 74(7-8), 519-526. doi:10.1016/j.ijmedinf.2005.01.003

Mandl, K. D., Overhage, J. M., Wagner, M. W., Lober, W. B., Sebastiani, P., Mostashari, F., Pavlin, J. A., Gesteland, P. H., \& Treadwell, T. (2004). Implementing syndromic surveillance: A practical guide informed by the early experience. Journal of the American Medical Informatics Association, 11(2), 141-150.

MAXQDA10. The art of text analysis: Online tutorials. Retrieved October, 2010, from http://www.maxqda.com/service/tutorials

Maxson, E., Jain, S., McKethan, A., Brammer, C., Buntin, M., Cronin, K., Mostashari, F., \& Blumenthal, D. (2010). Beacon communities aim to use health information technology to transform the delivery of care. Health Affairs, 29(9), 1671-1677.

McIlwain, J. S., \& Lassetter, K. (2009). Building sustainable HIEs. Health Management Technology, 30(2), 8-11.

Morse, J. M., Noerager Stern, P., Corbin, J., Bowers, B., Charmaz, K., \& Clarke, A. (2009). Developing grounded theory: The second generation. Walnut Creek, CA: Left Coast Press, Inc.

Nigel, K., \& Horrocks, C. (2010). Interviews in qualitative research. Thousand Oaks, CA: Sage. 
Roulston, K. (2010). Reflective interviewing: A guide to theory and practice. Thousand Oaks, CA: Sage.

Rudin, R. S., Simon, S. R., Volk, L. A., Tripathi, M., \& Bates, D. (2009). Understanding the decisions and values of stakeholders in health information exchanges: Experiences from Massachusetts. American Journal of Public Health, 99(5), 950955.

Saba, V. K., \& McCormick, K. A. (2006). Essential of nursing informatics. New York: McGraw-Hill.

Safran, C., Bloomrosen, M., Hammond, W. E., Labkoff, S., Markel-Fox, S., Tang, P., \& Detmer, D. E. (2007). Toward a national framework for the secondary use of health data: An American medical informatics association white paper. Journal of the American Medical Informatics Association, 14(1), 1-9.

Saldana, J. (2009). The coding manual for qualitative researchers. Thousand Oaks, CA: Sage.

Shapiro, J. S., Kannry, J., Kushniruk, A. W., \& Kuperman, G. (2007). Emergency physicians' perceptions of health information exchange. Journal of the American Medical Informatics Association, 14(6), 700-705.

Shapiro, J. S., Kannry, J., Lipton, M., Goldberg, E., Conocenti, P., Stuard, S., Wyatt, B. M., \& Kuperman, G. (2006). Approaches to patient health information exchange and their impact on emergency medicine. Annals of Emergency Medicine, 48(4), 426432.

Silverman, D. (2006). Interpreting qualitative data. Thousand Oaks, CA: Sage.

Smith, P. C., Araya-Guerra, R., Bublitz, C., Parnes, B., Dickinson, L. M., Vorst, R. V., Pace, W. D., \& Westfall, J. M. (2005). Missing clinical information during primary care visits. JAMA, the Journal of the American Medical Association, 293(5), 565571.

Technology Informatics Guiding Education Reform (TIGER). (n.d.). Designing usable clinical information systems: Recommendations from the TIGER usability and clinical application design collaborative team. Retrieved May 15, 2010, from http://www.tigersummit.com/Usability_New.html

The National Alliance for Health Information Report. (2008). Report for the national coordinator for health information technology on defining key health information technology terms. Retrieved February 19, 2011, from healthit.hhs.gov/portal/server.pt/...0...0.../10_2_hit_terms.pdf 
TN Office of eHealth Initiatives. (2009, Prepared June 30). State of Tennessee Advancing statewide eHealth efforts version 2.0. Retrieved September 21, 2009, from http://www.tennesseeanytime.org/ehealth/advisoryCouncil.html

Vest, J. R. (2010). More than just a question of technology: Factors related to hospitals' adoption and implementation of health information exchange. International Journal of Medical Informatics, 79(12), 797-806. doi:10.1016/j.ijmedinf.2010.09.003

Wright, A., Soran, C., Jenter, C. A., Volk, L. A., Bates, D. W., \& Simon, S. R. (2010). Physician attitudes toward health information exchange: Results of a statewide survey. Journal of the American Medical Informatics Association : JAMIA, 17(1), 66-70. doi:10.1197/jamia.M3241 
APPENDIX A. LETTER OF INTRODUCTION 


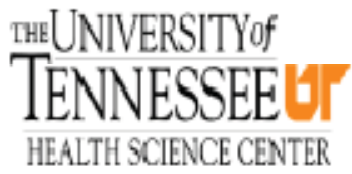

\author{
Principal Investigator \\ Shirley A. Thorn, MN, MAdmin, RN, CHE \\ UTHSC PhD Candidate \\ College of Graduate Health Science \\ Preparation Date: Sieptember 7, 2010
}

\title{
LETTER OF INTRODUCTION
}

I am conducting research for a dissertation and other publications on the topic of "Emergency Physicians' Perspectives on the Usability of Health Information Exchange". A health information exchange is defined as the patients' electronic health information that is shared between providers. I would like to ask you to volunteer to be interviewed.

The purpose of this study is to describe your views of health information exchange. The interview will allow you to speak freely so that I can obtain an in-depth understanding of your views, if you use or do not use health information exchange in everyday practice, and the challenges or benefits of health information exchange.

The interview is estimated to last less than 30 minutes and will be kept as brief as possible. All interviews will be in the emergency department or other areas of the hospital. You can select the setting most comfortable for you. Repeat interviews occur as necessary to make sure I have captured the views of emergency physicians.

Please be assured that every effort will be made to keep your information confidential; however, this cannot be guaranteed. The interview will be audio recorded to minimize note taking, but no identifiers will be placed on the audio recordings. You will not be identified in the resulting dissertation, publications, or presentations resulting from this research study. You are entirely free to decline to answer particular questions, or discontinue your participation at any time and the audio tape will be destroyed. There are no direct benefits or foreseeable risks to you for participating in this study.

This study can increase the understanding of what emergency physicians view as important factors in using health information exchanges and how they use health information exchange capabilities in care decisions. It is important to understand from the view of the emergency physician what works and does not work, and why physicians are adopting or not adopting the capabilities of health information exchanges.

Any inquiries you may have concerning this study should be directed to me at: sthorn@uthsc.edu.

Thank you for your participation and willingness to share your views.

Yours sincerelly,

Shirley A. Thom

This research study has been approved by the University of Tennessee Health Science Center Institutional

Review Board. You may contact Terrence F. Ackerman, Ph.D., UTHSC IRB Chairman at 901-448-4824 or visit the IRB website if you have any questions about your rights as a participant in this study or your rights as a research subject.

http://www.uthsc.edu/research/research compliance/RB/participant complaint.php 
TIMNEESTYA IRB NUMBER: 10-00974-XP

INNASHE IRB APPROVAL DATE: 09/09/2010

IRB EXPIRATION DATE: 09/07/2011 


\section{APPENDIX B. INSTITUTIONAL REVIEW BOARD APPROVAL}

\begin{tabular}{lr}
\hline THE UNIVERSITY OF TENNESSEE \\
Health Science Center \\
\hline \\
Institutional Review Board \\
Memphis, TN 38163 \\
Tel: $(901) 448-4824$
\end{tabular}

September 9,2010

Shirley Ann Thorn, MN, MAdmin, RN

College of Nursing

Department of Nursing

620 Lamar Alexander Building

Re: 10-00974-XP: Emergency Physicians' Perspectives on the Usability of Health Information Exchange.

Dear Ms. Thorn,

The Administrative Section of the UTHSC Institutional Review Board (IRB) has received your written acceptance of and/or response dated September 7, 2010 to the provisos outlined in our correspondence of September 7, 2010 concerning the above referenced project. The IRB determined that your application is eligible for expedited review under category (6). In accord with 45CFR46.116 (d), informed consent may be altered, with a cover statement used in lieu of an informed consent interview. The requirement to secure a signed consent form is waived under 45CFR46.117(c) (2). Willingness of the subject to participate will constitute adequate documentation of consent. The IRB has reviewed these materials and determined that they do comply with proper consideration for the rights and welfare of human subjects and the regulatory requirements for the protection of human subjects. Therefore, this letter constitutes full approval by the IRB of your application and letter of introduction dated September 7, 2010 [stamped IRB approved on September 9, 2010]. This study was approved for 12 months with an expiration date of September 7, 2011.

This study may not be initiated until you receive approval from the institution(s) where the research is being conducted.

In the event that subjects are to be recruited using solicitation materials, such as brochures, posters, webbased advertisements, etc., these materials must receive prior approval of the IRB. Any revisions in the approved application must also be submitted to and approved by the IRB prior to implementation. In addition, you are responsible for reporting any unanticipated serious adverse events or other problems involving risks to subjects or others in the manner required by the local IRB policy.

Finally, re-approval of your project is required by the IRB in accord with the conditions specified above. You may not continue the research study beyond the time or other limits specified unless you obtain prior written approval of the IRB.

Sincerely,

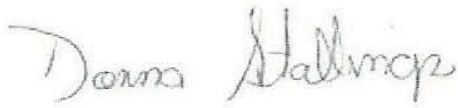

Signature applied by Donna L Stallings on 09/09/2010 09:06:55 AM CDT

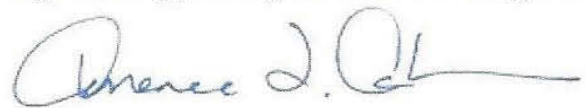

Signature applied by Terrence F Ackerman on 09/09/2010 09:09:00 AM CDT

Donna Stallings, CIM

IRB Analyst
Terrence F. Ackerman, Ph.D.

Chairman 


\section{APPENDIX C. CODING SYSTEM [1132]}

The following code system, which is the assignment of code to text, was extracted from MAXQDA as developed by the researcher.

\section{User of HIE [59]}

What does it mean to the emergency physician to be a user of HIE?

\section{Non-User of HIE [94]}

What does it mean to the emergency physician to be a non-user or reduced-user of the HIE?

How does the emergency physician describe being a reduced-user?

\section{Experience [37]}

How long has the emergency physician been using the HIE?

Do they have experience in other emergency departments or exposed to other HIE systems?

Do they currently work in more than one emergency department- that is different sites and therefore exposed to different documentation systems and health exchange capabilities?

What is their experience with EHRs?

Are they using hybrid systems, especially if working at more than one facility?

\section{Usability/Frequency of Use [43]}

How frequent does an emergency physician indicate they use the HIE?

Frequency: Low user, medium user, high user?

Percentage of times HIE used?

\section{Typical Day [59]}

What is a typical day like in the emergency room?

How is the HIE used or not used?

\section{View Chart Before [2]}

When are emergency physicians viewing the HIE, before or after seeing the patient?

How does the timing of viewing the HIE affect making clinical decisions?

\section{Information Currently Contained in HIE [42]}

What information is currently contained in the HIE?

Inadequate Information in the HIE [80]

What information would emergency physicians like to see in the HIE?

What information would emergency physicians like to see added to the HIE?

Gaps or missing information?

Changes needed to improve the HIE? 


\section{Care Decisions [102]}

How does using or not using the HIE impact how emergency physicians make care decisions?

Does using the HIE change how emergency physicians make care decisions? Yes/No

If yes, how does using the HIE impact the emergency physicians' care decisions?

What impact does not using the HIE have on care decisions?

\section{Challenges or Barriers to Use of the HIE [113]}

New: What challenges or barriers do emergency physicians experience when using the HIE?

Previous: What are the challenges or barriers for emergency physicians to the use of the HIE?

\section{Equipment [25]}

What issues with equipment impede or promote HIE use?

Are there enough computers in the department?

Portable computers?

Documentation in the patient's room?

Access challenges?

\section{Success Factors [58]}

Why is the HIE successful?

What is needed to make the HIE successful?

\section{Changes Needed [138]}

What needs to change in the HIE?

\section{Efficiencies with the HIE [112]}

What efficiencies/benefits do emergency physicians experience with the HIE?

Does the HIE give emergency physicians efficiencies?

\section{Training or Education [54]}

How much training or education did the emergency physician receive to use the HIE?

Opportunities for the emergency physician to give feedback about the HIE?

Ongoing education about the HIE?

Current View of HIE [77]

How do emergency physicians currently view the HIE?

\section{CT Scans/Headaches [2]}

How do emergency physicians view ordering scans?

Ordering of CTs for patients with chief complaint headache?

\section{Confidentiality Issue: Non-User [1]}

How do emergency physicians view confidentiality issues? 


\section{Emergency System [29]}

Comments about the emergency system, which may be related to the HIE or not related. Comments include issues, problems, or positives.

\section{Revenue [5]}

How do emergency physicians view revenue issues related to non-use? 


\section{APPENDIX D. ADDITIONAL EXEMPLARS}

The following are additional exemplars of each of the study themes, including the antecedent of a typical day, which augment and solidify the researcher's theoretical interpretation and analytic explanation of the phenomena of HIE usage among emergency physicians. The exemplar quotations of the participants are presented in italics and have been edited for readability. Ellipses indicate omitted words or sentences and brackets denote the researcher's insertions for clarity. Identifiers have been removed to protect anonymity.

\section{Antecedent Condition: Typical Day}

There are probably many different reasons [for frequent flyers using the emergency]. Some are probably looking for a warm bed, place to sleep, and a meal. Some, they have to pay a co-pay to see their doctor and it is free care here. For a lot of people, it is a free ride in and free care because they know they are never going to pay the bill.

Today was lousy with that. Where everyone was like, I couldn't get into see my doc today because it was Friday and all the offices are closed for the holiday. So I think that having a primary [provider] would decrease some of what we see but not to a large extent because I have seen probably four or five patients [today] that have seen their doc for the same problem within the last week and a half. I am still coughing or I am still in pain.

Sometimes just by the complaint. The complaint is just so stereotypical type, I'm from out of town, I got chronic pain, and it is just some issue like that.

[If I was] in charge of the health care system, there would be 15-20\% of these patient that would never be allowed to enter the door. I would have them cut off up front, maybe higher than that and say, you don't have an emergency we're not seeing you. I would have the ambulance drivers, the paramedics, be able tell patients you don't have an emergency we're not going to bring you. And there was a time when things were that way. But not anymore, but I'm not in charge of that so I live with it because otherwise I would just be miserable in my own skin so I have accepted that- that is my formality that they are going to order tests on patients and they are going to order a lot of them that are unnecessary and I'm through you know being terribly upset about it anymore, because I can't stop that train, it's out of control, its running downhill, and there are no breaks on it.

[Emergencies are now displaying wait times on the internet for the public?] Yes. I think that is ridiculous too. That's a ridiculous concept because what person who really has an emergency, what paramedic that is picking up somebody who has been in a roll over motor vehicle wreck that has a gaping hole in their chest is getting on the internet finding out which place is going to see them fastest. They're going where they need to go. And 
that is because patients who really have emergencies are getting seen and patients who may or may not have emergencies might have to wait and I think that is OK.

[Using the HIE] Absolutely, whenever the patients come in that were found down by their family, particularly if they don't use our facility most often. They often have their records somewhere else. Sometimes helpful, sometimes not, just depending on the patient, but I always look.

I guess if we had somebody just show up comatose, probably not, if somebody said yea, they were in at the [another hospital] last week for the same thing you would probably try to look something up [in the HIE]. But not on everyone.

\section{Accessing the HIE during a Typical Day}

A lot of it has to do with how frequently, when was their most recent visit, and where was the most recent visit, and those sorts of things. It is not a well-defined criteria in my mind, I just play it by ear as to what the patient tells me, what I need to know, and how can I get that information.

We want to look first at our information, see if they are our patient, did they come in frequently here, if they don't come in frequently here, then yes, I am going to look to see if there are other records available.

\section{Timing or Specific Needs}

Well, what usually will happen that triggers my use is that they'll come in and I'll either want to check and see if they have been in some place recently or if they tell me that or if I just think that they have. That's usually, or they're returning for a visit. If I think they've been somewhere else I'm just checking to see or they tell me I had a CT scan done [elsewhere] and that kind of confuses me too. They'll go to one facility, get a work up done, and then they'll decide two days later to go to another facility. That happens very frequently. If you're able to get that history, I'll go to the HIE ... and get the information I need.

I go see the patient, I talk with them, I get a history. If I look in the record, I just don't see the record until I visit the patient. Ninety percent of the time I will ask them, have you had this problem before or have you been in the hospital, have you had this test done before I pull the records.

Some people will just pull it up to see if somebody has been somewhere else for whatever reason. They're seeking drugs or something like that. If it is something, I think that if here they said that they came in with abdominal pain. They've said, I was [elsewhere] two days ago and I had all these tests done. Well I think yea, that makes sense to look it up and do that. 
I don't just pull the record at random. The only time, I hate to say this, I am on the HIE already looking at the patient. I am already seeing and I go back to the home page and I am going to see the next patient. I see they have 417 ER visits at another hospital, and 280 some visits here. Well, I am going to go to the room and see what they have in here, if it is what they have been having all these years.

\section{Information Contained in the HIE}

Labs and radiology results, and if there's a discharge summary that's a bonus.

Hone in on her chief complaint as to what happened at other facilities that she may have been to.

[Would you like to see the actual EKG tracing rather than just the report?] Absolutely, that helps a lot. An actual picture of the EKG, very subtle changes can make all the difference in the world. [Same as the radiology report, would you like to see the films?] It's not as important as an EKG. You can usually know what you need to know by getting the radiology report. They usually hymn and haw enough that you can get an idea, because it's not quite as important as it is for us. Now a neurosurgeon may say entirely different than me. It is [what] different levels for different specialties will require but from the ER standpoint to me the ER record, discharge summary, medications, lab tests, and EKG. Results basically.

We have lab, radiology, discharge summaries, $H \& P s$, it's perfect.

\section{Theme 1: Using the HIE}

I describe [the HIE] as information systems that give me access to a patient's past medical history or their medications or other modalities that will help me best take care of the patient.

It's a database for patient information and for me in particular, an HIE means outside the [internal EHR] system because we have our own internal health information system.

I have never seen ER physician notes. I have seen procedure notes, discharge summaries, sometime H\&Ps, but that's mostly what I get. I don't think ER notes.

Sometimes you think you may get a discharge summary, sometimes.

\section{Characteristics of a HIE User}

I think it [the HIE] aides in the quality of life for the patient. Because they're not medical personnel, you can't expect them to know what all their diagnoses are and what each 
physician said to them. They're not going to remember it.

So it really I mean, looking back, how we did this years ago trying to find out what our patients [had] communicated to them. To me it's mind boggling that now if I don't have [the HIE], it's like my arm's been cut off without surgery. You're going blind but having this information has changed the way we ER physicians do practice. [The HIE] all comes from the center location and we have access to it and that's great. It's like wow, you know I won't miss anything, I won't feel like I'm going blinded. I know exactly, to the best of your ability you can find out things about these patients that otherwise you wouldn't have access to.

Well yes, [the physician] said something about that, but a lot of people it doesn't mean the same to them, but to another medical professional, it means a lot to us. What another physician thought, what another told them, why they switched this medicine to this medicine, it means something to us. Whereas patients may get it, they may not get it, and this takes that guess work out of it.

I am interested in knowing if it applies to decision-making that I need to make. So, I use it on a selected basis. It is very important for certain patients who have a medical history that impacts the way that I am going to treat them here and they have no records that I can refer to in the emergency department. I guess that is the most important category, the patients that I see where we know nothing about them here. The patient is unable to give reliable information, they have been to other places that have information loaded into the system, and so I access it to gain knowledge about information that is otherwise unattainable or would take days or hours or be very difficult to obtain. The other category of patients I use it in are those that have frequently visited the emergency room and there is suspicion about their motives of being here. I am able to find out information that the patient is willingly withholding from me, in order to obtain something and in most instances either that means to get drugs here or because they have health care problems that they would otherwise not want to talk about for whatever reason, like drug usage, illicit drug use.

If they were told at some hospital that they need to follow up and get an outpatient EGD, but they never did ... They say, well I tried to call this place ... then you can get a caseworker involved ... They're still having the same problems, but they didn't follow-up so we can help them with an appointment. That helps a lot, trying to just take care of the patient. For me, to try to navigate the health care system is hard, and for our patients it is even harder than that. If they don't get any results, they come back to an emergency room. I think it helps... All the pieces to put together.

I think [the HIE] is a good tracking for where patients go and what they do and how irresponsible patients are. I think it is good to know.

I think that a lot of the patients that I'm looking up on the HIE are patients that are either self-pay or Medicaid, because those are also the people that generally tend not to have primary physicians or if they do have a physician that it's difficult for them to see him because of problems with co-pays etcetera. 
It's depending on the scenario of what the complaint is. If it's a person with multiple visits here with abdominal pain with the same theme, I'm trying to see what they have had done recently, trying not to repeat or what have you, and also trying to gather information from those who we can actually be more efficient with what is going on.

The good thing is you can look up records from other hospitals and see where people have been, what studies they've had, because a lot of times someone will go to the hospital down the street yesterday and will show up here the next day and have the same complaint. You can look it up in the HIE and find out everything they had. They had extensive workup and it was all negative and their complaint really hasn't changed greatly, you cannot repeat all the tests. Not do the two million dollar workup.

It is because our people are from a different hospital that show up in our ER from ... other places. So we have information that I can look it up, their labs, what was done recently, or was a cath, or discharge from the previous place, which otherwise without the HIE I would not have been able to do it.

[The HIE] is great for not duplicating tests, if someone just had it all done recently, it's great for trying to weed out for someone that's drug seeking, or other reasons. I would love to see it easier to use.

I learned to practice medicine by obtaining information and making a decision and using laboratory and radiographic information to either confirm what my suspicions were or to seek information that I might otherwise not be able to get that's going to have a big impact on the patient. Doctors now days, including lots of my partners, before they go in the room they've already searched the HIE system. They've looked at it, they've searched it, they know all about the patient before they get in there to me that's kinda biases your opinion before you go in and it flaws the logical process of decision making because you already know what you think you know before you go in to see the patient.

\section{Experience Using the HIE}

I don't know how much more I would use [the HIE]. I think I probably would use it more because it would be easier to. I guess achieving things right now that I have been looking at it so they [the nurses] pull it up for me so while their working I'm seeing patients so I can come back and decide and send it to them.

Well, yea. I mean it [the HIE] is nice to have. Occasionally I can make clinical decisions based on the information I get off of it by how much workup I do or do not want to do. And how much investigation of the complaints I'm going to do. But there is quite a bit of information you usually [have] already in the system here [internal EMR] and a lot of these patients have at least been here enough that you can glean from just the visit notes from [this ER] as to what's going on with them. That, it wouldn't be the end of the world to me if it [the HIE] was gone tomorrow, but is it useful, yea. Am I glad it is there yea. Do I use it that frequently no. 


\section{Who Has Access}

[Residents] use it. They jump right in because they like to, if a cath report, they like any other tests that the patients have had done at other facilities. They are coming down to the emergency room, and saying [to the emergency physician], I admitted this patient the other day and the doctor that worked the last time forgot, because what we usually do when I go in and I see a patient has been somewhere else, if I am going to admit them, I print off the main things that I consider, CT reports, cath reports and I attach them to the chart. When they go upstairs to the floor, the doctor that is taking care of them can have access to the information that I got from these other facilities. Sometimes, we forget. The resident will come down and say can you log me in and get this information. And we do.

Because one of the internal medicine attending used to do some moon lighting in the ER and he uses [the HIE] when in the ER, but he just doesn't use it when on the wards. Why, I don't know.

Any other nurse who really wants it in the ER also are granted access but most of them don't have the time or the inclination to do that. Chest pain nurses are a different story. They have been taught to look and they bring that forward to me without normally me having to ask for it. Occasionally, I say is there anything in the HIE or is there any records and they'll say we're looking but we haven't found it or I'll look for you ... Some ER nurses who have made an application to get access but not all have made application; in fact, very few of them probably have made application because I don't know if it's any particular benefit to them.

All my nurses have access so they would access the information for me and then print off what we need ... Usually I just find one per shift [nurse] and they generally pull it up for me ... [And all the nurses have access to it?] I don't know about all of them, but typically from my experience up to this point working over here, at least the charge nurse has access to it and have been able to pull it [the HIE] up.

Actually the physicians are the only ones that can [access the HIE]. It's only on our login and actually only the ones that are attending physicians. In other words, residents don't have the capabilities of doing it. It has to be, its guarded pretty closely and only emergency room physicians, like the other internists in the hospital don't have the capability.

Because we don't have accessibility between the consultants as far as the [HIE], most of the radiation involved with CT scans, which is our biggest thing, because they don't have access to it, that means we are still going to do CT scans.

\section{Theme 2: Influencing Care Decisions}

The information you glean can certainly change what you do. There's no doubt it has an impact. 
I think I use it [the HIE] a lot and so it helps me proportionally, maybe it helps me maybe once a day. But if I was only looking to it a few times a day, then I may go several days without it helping me. I can see that it is more a function of our whole system, not having all that we would want like outpatient physician notes would be very helpful.

[The HIE] changes what you are going to do for a patient.

I guess everybody's got their time management in different places and there is not a whole lot of downtime ... And I guess I would see [the HIE] as that is just one more thing that I have to do that will bog me down more, to do that on everybody. But again, having everybody's information, the more information you have on everybody the more it helps in taking care of.

\section{Missed Information}

[The HIE] helps in a lot of ways. There is a positive impact it does make in your decision-making ... Somebody with surgery from [another hospital] comes here ... I ask this patient who is the surgeon, I don't know, it is one doctor operated on me. I will pull the op note and all that and if there is any problem complication, I call the [hospital] instead of calling and shopping around looking for a doctor. A lot of times when you call the surgeon, they say, I didn't do the surgery. That is nationwide. I don't blame them. This is to find out who's the surgeon. $90 \%$ of these patients don't know who is their surgeon. I will call and say, this is what is going on with your patient and they say, send the patient over here. I don't sometimes have to repeat any tests. You know just by examining the patient and having that record pulled, all those guys say, just send him over here and we will take care of him and don't do anything.

With [the HIE] if they had abnormal EKGs, you know everything ... and if they had surgery at other places, you get access to that, it just helps you better manage the patient. And you know what you need to know.

Before we would miss a lot of the patient's problems if we didn't have [the HIE] because we were just going on what they tell us and then after some days later you realize, oh yea, they had a history. I can remember once talking to a family member about a patient two days after I saw them. They [said] did you know that she had a stroke in the past? I'm like no, I didn't know. That would have been good to know two days ago.

\section{Assortment of Clinical Decisions}

Whenever I have a question about somebody, if I'm interested in visits to other places, what all has been done workup wise at other places, are they frequent flyers, do they pass around all the emergency rooms, are they drug seeking, if they've taken chronic medical conditions that have required multiple CT scans and stuff. I want to know if they have had a bunch of work-ups in another hospital, so we don't repeat all that stuff over here. 
I am interested in knowing if [the HIE] applies to decision-making that I need to make. So, I use it on a selected basis. It is very important for certain patients who have a medical history that impacts the way that I am going to treat them here and they have no records that I can refer to in the emergency department. I guess that is the most important category, the patients that I see where we know nothing about them here. The patient is unable to give reliable information, they have been to other places that have information loaded into the system, and so I access it to gain knowledge about information that is otherwise unattainable or would take days or hours or be very difficult to obtain.

We have a lot of patients, drug seekers, that come through the emergency room and if you know that they have been to [another hospital], or somewhere like that, and just got 12 Percocet ... because patients will say, no they didn't give me anything for pain. But, you're like, oh yes they did. So that helps a lot too, because a lot of patients don't realize that we can communicate with other hospitals.

\section{Tracking, Comparing, Trending}

For certain patients, [the HIE] does make a huge impact. It allows you to spot trends that you wouldn't otherwise have any way of finding. Without the [HIE] I never would have seen a guy had four different names in the system and he actually did give a story of he had been at [another hospital] and had this work-up done, and I looked up, and under a different name he had indeed... So that went from the guy who would have been admitted, cross sectional imaging, GI consult, to this is the same pain you have been having all year. You've had every test that we have to try diagnosis this and we are not finding it, here is some nausea medicine, go home. That is one patient that shift that made a huge difference on the saving to the health care system-was probably somewhere in the 12-15,000 dollar range by not admitting him and not working him up again for the same thing he had been worked up for multiple times before.

No. I never have pulled up any graphs.

[You do get graphs?] Yes. But it is not always, and I don't know what makes the difference. Maybe it is older information or fresher information I don't know ... Like sickle cell patients. You know it nice to see they have been in the high 20 s all along and here is a 16.

It would be nice to be able to graph it to see trends and data, but that takes another level of integration that we currently don't have.

\section{Reaction Time}

[The HIE] has helped everyone here-made it easier for us to find out studies that have been done on patients. It's easier for us to find that and reference current lab values to 
previous lab values. It changes what you are going to do for a patient. You can tell if a patient is chronically sick...It tells you how fast you need to act on a patient. The patient comes in for chest pain and you look them up and they had a heart cath at this hospital three weeks ago. You know that's clean. You don't have to rush and admit the patient. So it is beneficial. A lot of the patients we see won't remember what was done to them. It's limited for now on what you can do. We can't look at all the histories of that but we can at least on critical things. It is also good in detecting drug-seeking behavior. We're in an area that we see a lot of recurring patients coming in for ulterior motives. It can help us determine whether we need to do anything further, [or] just follow up with their primary care doctor.

We get transfers here all the time from other hospitals. They come here from [other facilities] or somewhere, most of the time for whatever reason if they got a head bleed and they don't have a neurosurgeon and they will come here. We will pretty much go with their tests. Now sometimes the neurosurgeon will repeat something or in just whatever case, sometimes the doctors will want to repeat everything. But for the most part we will, for me I go with what other hospitals have. Now if it's something like a potassium or something that can change from day to day, there's something like that, you may repeat. But you know for the most part, we rely on what other hospitals, what their ratings are or whatever.

You would be surprised that they send them with one piece of paper from the nursing home that don't even have their medications.

\section{Amounts of Radiation}

There have been times where I have said, this patient just had a CT scan at [another hospital] and had a CT scan of the head and just had it yesterday. I'm not going to rescan them. I will document on the medical record that I reviewed the results of the CT scan from the medical record from [HIE].

I understand that there are some tests that you cannot replicate like a procedure, like someone had a head scan done a full five days ago at [another hospital] and I am not too compelled to do a repeat one when I don't think they need one. So I just look it up. It [the HIE] helps me out.

Like I said, I don't use it on every patient. My curiosity doesn't drive me to know everything about every patient that comes in here. If it doesn't have any impact on my decision-making, I just as soon not know. Because I don't have to know. And I don't have to know everything about every patient I see here. I don't have to make a diagnosis on these patients down here. I have to know if they have an emergency. If they do, I got to treat it and then I got to decide if they could go home or have to stay in the hospital and all this information is not always necessary for me to make that decision. 
I go by the patient's history even though I know they have been somewhere. I keep that in the back of my mind of course, but I always try to say, $O K$ what is different today and maybe two days ago the same symptoms, if I repeated the same tests it might show something today, and it didn't two days ago. So, if I think it is necessary, regardless of if they had it recently or not, I'm going to order that test.

\section{Ordering Tests}

I can use [the HIE] to form my opinion of what I am going to do. It doesn't necessarily, sometimes it changes, but like I said it's just kinda more information that I have. More than I am looking for. Everything I need from them, I am not going to do. I treat the patient and not the numbers, not the results, but it helps me to treat the patient.

I think [it is] very useful from an ER standpoint to be able to say $O K$, I know what's going on with the patient, what has been going on with the patient is this just the same, is this different, what do I need to repeat, what's already been done.

I had a patient I thought needed a MRI for a C-Spine and she didn't tell me she had one done two weeks earlier at [another hospital]. But I found that out because she told me she had been going to the [other hospital] so I went and looked. And sure enough, there was a MRI. So I cancelled the MRI.

We've looked at reports of stress tests for the chest pain center where you think you need a stress test and it hasn't been done and they don't know what you're doing. Well, it's been done within the last six months, and there is no point in repeating the stress test. Either we're going to get cardiology to cath them or treat them non-interventional wise.

[The HIE] has helped me to take care of my patient properly. Helped me avoid repeating diagnostic tests because we see the most common things here is people go from one hospital to the other. It has helped me make proper decisions to take care of my patient because like I said, if you already had an imaging study, if I need to do another one it will help me. If I need to call a surgeon. I meet the patient, do I need to do further testing. It also speeds up the process. You used to call the hospital and get medical records. Call that night, nobody is there. You know it just is very efficient to me. It helped me a lot.

A patient comes into the chest pain having typical or atypical chest pain so that they went to the hospital before or have not. We will just check out to see if they have had an evaluation with a cardiac cath, stress tests, and things of that nature, so we will not have to repeat everything and evaluate from that standpoint. That is how we do it by just looking at what kind of work-up they had at a previous hospital with regards to the chest pain and things of that nature. 


\section{Concept of Life-Saving}

It's [the HIE has] been life saving, I mean often times you will have somebody that will come in that the only thing that they have, maybe completely comatose, and the only thing they have is their drivers license. I can pull up their last name and social security number and immediately be able to figure out what's going on with this patient and where they've been and what's been going on with the patient. So that's absolutely, it's like I said, life saving ... [What do you see are the benefits, of the HIE, for your care decisions?] It's huge ... I'll give you a classic example. When somebody presents with a heart attack, the ambulance takes those patients to the nearest hospital. It may not necessarily be that patient's hospital. That patient maybe having a heart attack. The ambulance crew will take them to [names hospital]. Their doctors may be here, but they will take the patient to [the closest hospital], because they're having a heart attack. They have an acute life-threatening problem and that's the right thing to do. But that doctor has to have access to all the information here in order to be able to say, where is the patient's last cath? What's been going on with the patient? Although the history from the family is great, we need more than that as physicians. We need to know the specifics of what is going on. We have to be able to see what's going on. Now in the old days, we would fax a letter of clearance and talk to medical records, and maybe in the next three or four hours we might get something involved in with an H\&P from 1997. But to be able to have that information at your fingertips right away, that can be lifesaving. And it has been. [Have you ever seen an instance where not going into the HIE produced a negative outcome for a patient?] I have not. I can image where I would but I haven't seen it. I mean, I can image that you would certainly have to have that. But, I haven't been made aware of anything where not going in would have cost a patient a bad thing.

\section{Theme 3: Struggling with Challenges and Barriers}

But for me,[the HIE] is kind of cumbersome, it is time consuming, and that is why I don't use it as much as I would probably like.

I think navigating it is not intuitive. You will sign up for somebody and you will click on a patient and then retracing your steps is kind of cumbersome. You have to clear out the screen, go back into the scroll, and find the patient.

If it's too cumbersome, it's not going to help you.

\section{Difficult to Access}

It's just one more step trying to get to [the HIE]. Now it is more clicks and more time and so that keeps me from using it more than I guess in the sense that like others are. 
If you can cut down clicks, you'll use it more. That's an ongoing issue internally not just with this particular problem, it's also within our own programs. Once they change things, if they make more clicks we don't use it as much.

Ideally, we need a single sign-on interface with our system, so that once we log on my system, it automatically allows me privileges. And the other thing, there is a time out screen that if you don't use the HIE, then you have to log back in. That's a burden for a busy ER...The [HIE] is down to 15 minutes and if you haven't touched that screen in 15 minutes, then it logs you off. [You're not single sign-on yet?] Not with [the HIE]. It is not incorporated into our log-on ...You go into the screen where you log in and all my icons are there. My passwords are remembered so I don't have to enter a password or every little app that I need. I can get to [the HIE] from within my chart but I still have to enter my user ID and my password every time I access [the HIE].

You had to have that token to be able to get on and you also have to have your own pin. It was really not user friendly. As I said, the key in all this whether it be EHRs or HIEs is to have an effective user interface. They did not have an effective user interface and so the actual utilization of that was pretty low. Most of the hospitals were running about $7 \%$ to $10 \%$. Well, we said this is no good. We don't need to do that so the Director actually came to me and we kind of worked through some stuff and now we have it where it is seamless into the EHR through basically point and click. It's based on every hospital's normal login so whenever we get into the EHR by our normal individual login, it's an icon on our screen so we can immediately pull it up ... We actually process mapped the whole thing. We looked at every single thing and looked at every click and you know we've got it down to five to be able to get that. It's pretty good. We can't go anything more than that but we have gone from like 14 to five. A lot of that is bringing in single sign-on as a vendor to be able to deal with this and do all those clicks we normally had to do. We got to one point where we had to sign on twice in the same thing. You had to signon and then you had to sign-on again. Why would you have to sign on twice, you just signed on? And that's the thing from a physician standpoint, when time is crucial especially in an emergency department, time is crucial. You have peoples' lives on your hands. You have to have that information and now. Those 12 to five clicks, make a big difference. Also, anytime you have that you decrease your chances of failure and you increase your compliance. So those are absolute, you got to have those.

Ideally, we need a single sign-on interface with our system, so that once we log on my system, it automatically allows me privileges ... [You're not single sign-on yet?] Not with the HIE. It is not incorporated into our log-on. When I say single sign on you know what I am talking about? You go into the screen where you log in and all my icons are there. My passwords are remembered so I don't have to enter a password or every little app that I need. I can get to [the HIE] from within my chart but I still have to enter my user ID and my password every time I access the HIE.

Too many phases, interphases. Just once like I said. If I can just log into this system [internal EHR ], and here now, I have to go to this and look for the HIE, and log into that. 


\section{Privileges and Access}

It took me awhile to get through the paper work and get my own password. So after a couple of months I really used it [the HIE] a lot. Actually very, very helpful.

Some of the hospital physicians have access to it. I don't think they use it as much as we [the ER physicians] do though ... they did get a couple of the internal medicine hospitalists access to it, but they tend not to use it a lot.

The midlevels do have access. We have nurse practitioners and physician assistants ... Nurses don't have it, consultants don't have it, nobody else has it.

[It is only the ER physician using it and Nurse practitioners?] And midlevels, yes, and PAs, that's it. Even the medical students don't have access to it, I have to log them on. [Do the nurses have access?] I believe some do. I don't know if all them do. Now I know the charge nurses do because they sometimes will go print some stuff off for us as well. I believe that they do, but I do not know to what degree or what extent.

[If you admit a patient, do you know if the physicians on the units, the specialists that are seeing a patient, do they have access to the HIE that you are aware of? ] I do not know.

If we find something that was pertinent to what we were doing, we print it off ... It stays on a paper chart ... I think we as a user just throw it in the shred box afterwards.

I think it [printed material from the HIE] just stays with their chart. Or, we are just using it and we will shred it. I do not think it goes, unless perhaps there is a discharge summaries or something that we will specifically print off by request and we will send that with them. The hospitalists will get that information to print it off and carry it with them. I believe they use it as well.

When I am admitting, most of the time I will print something that is relevant.

\section{Gaps in Information}

Sometimes a patient will be at [another hospital] and we'll get a discharge summary from the hospital but won't get an ER progress note.

[You had to go into the HIE to find out there isn't a record?] Yes ... somebody had surgeries at a lot of places, you see gaps. Doctors have not done the dictation, the discharge, op note, all that stuff. If there is any delaying that to you, you won't see.

Some [hospitals] update [the HIE] the next day. If they go to a hospital now and come straight over here, I might not be able to see what they did. I'll see that they've been there but won't be able to see what they did. I think [two of the hospitals] update the 
same way we do within a couple of minutes. I think maybe one of the other hospitals, I don't know which one, updates several hours or day after.

Sometimes the notes from other facilities are kinda hard to read through.

I think [the HIE] is easy to use. I don't know if it's the reporting, whether all hospitals use it, because a patient has told me I had a cath and seems to be a reliable person, and I can't find the cath. So you know not all the information is there and I realize that. I just try to use it in the sense that I can try not to repeat things.

I wouldn't mind seeing [the HIE] expanded to some of the outlying hospitals in the region.

[The Veterans Affairs Hospitals] are not on the [HIE] system-that would be nice.

It would be great if this were a nationwide system instead of just the hospitals in this locale. Somebody just moved from California where they have been to the hospital every day.

\section{Design Flaws}

Yes, I wish I could keep [the HIE] up. That's the problem. I hate having to sign in, it is just the same problem we had when we first started having computerized data entry. We would come and leave the computer and for privacy reasons they said, well we can't let the computers stay on. [The computer] has to turn off, you got to sign back in. Well that is not conducive to emergency medicine. It may be to the floor where a person may leave that station and go someplace else and have forgotten to sign off so the computer's still open. But here, I'm gone for a matter of minutes and I'm coming back. I have to sign in every time that I need access is very cumbersome and it is not conducive to the smooth flow in operations and expediting flow through the emergency room.

Why aren't they giving me the same courtesy, or extension of privileges that I have been granted here [for the EHR]? They say they can't do that. I say, even this little bit of information tells me a whole lot as long as I don't have to log in here, I don't have to waste my time to look at this chart [in the HIE].

It logs you out, it is a timer, and that is OK with this. I am OK with that. I'm fine with it. I don't have to go back and forth there, I will just look at [the HIE] once, and then I am done.

[The HIE] stays for a certain amount of time on there and then it logs itself out for security reasons ... Most of the time, by the time I am done with it within five or six minutes, it is automatically logged out. [About a 15-minute time?] Don't know. I can't tell you. I don't know how long [the HIE] stays logged on because I've never counted but I can tell you that most of the time by the time I am done with it, it's logged out. 
I think navigating [the HIE] is not intuitive. You will sign up for somebody and you will click on a patient and then retracing your steps is kind of cumbersome. You have to clear out the screen, go back into the scroll, and find the patient. The way it is set up, it is a very small area. There are maybe 50 patients and there is only two or three on the viewer area that they have. You have to click on it and scroll through 15 to 20 names and watch it fly by and look for the name to come up. I have played with it here and I've tried to increase the size of it but I think there's a limitation of four to five inches. The names will come flying by and sometimes I will miss it and have to go back. The benefits of having all those names entered in, the current patients entered into it, I think is lost a little bit because they didn't make the screen big enough to where there were 12 to 15 names on the screen and you could just scroll down. It would be easier to go through it. Also, I think the way to click on the laboratory data, again I suspect that this is because of the feeds that they have, is cumbersome. You'll click on one and you will get a BUN and creatinine. You will click on the next one and get the electrolytes. You click on the next one and you get the CBC. Click on the next one, you get the differential. So to get one set of labs from the emerg department visit, I might have to click on four or five different links sometimes. I would rather it all just dump into one screen and just populate that screen and let me see them all at one point.

You rush in on the last patient last seen here. You don't have time to go click it at another place, log in, scroll down, pull records. Like I said, if there is already an interface on this guy I'm seeing now, you can just click on it and get it.

Everything that is slow to me is painful to me no matter what it is. And I view this [the HIE] as slow, even though it is better than it was to start with so they are making progress but it is still not where I would like it but they are making progress.

\section{Physician Communication}

\section{Physician input and feedback}

I think [the HIE] could have been enhanced even more if there had been more end-user input during the construction phase than there was. I'm not aware that there was any to be honest with you. It is kind of like, here, use this when it was rolled out ... I would enlist some end-user feedback for upgrades to the system to make[the HIE] a little bit more user friendly or get specific comments like we would like a section for H\&Ps and discharges ... I know [the HIE] have a board and I think they probably have meetings. There's

probably opportunity to go to those meetings, although I haven't seen them publicized but I know they existed. There is probably opportunities to attend if you wanted to find out but there is no outreach to try and get some feedback.

If you ask computer guys to give you something and they give it to you, often it slows things down more, is my experience. So that is why I would probably say I don't have any suggestions for improvements to them if they had asked. 


\section{Education and training sessions}

But that maybe is nice if there is a way that I can back out of one patient and pull up another one. But it is not obvious to me because it has never worked. If someone could show me that, then that may help make me faster on [the HIE].

You need education. I think that is the biggest thing here. Of course, [the HIE] is a little bit cumbersome to use. If you could make it easy to access and you go out and educate doctors in the use of this. I think [the HIE] guys did a good job-signing people on but I don't think [they] did a good job educating people about this.

I think when we have gaps, I don't think it's a problem with the [HIE] system, I think it's a problem with the hospital putting the information in. Sometimes a patient will be at [another hospital] and we'll get a discharge summary from the hospital but won't get an ER progress note. I just think it's a problem with the hospital and not the system. I think we still need a lot of education with the people at the separate hospitals.

I think a lot of it is still an education issue. It's getting [the HIE] t back out there. There's new versions that are coming out every six months, which is great. But again, that's one of these things where we use those new versions, this new education tool for more of a refresher. We do that here. I know that they don't do that at other hospitals. They just kind of say, o.k. here is another version. Well, every version that we get we have them come in and we do a re-education on all the physicians and providers. Hey, look here's what it is, here's what new. And then they get feedback, what can we do to make it better. So I think you have to have that process or else it won't be utilized. You have to keep showing the physicians how this tool can help their patients ... If you have people that are over utilizers, why are you using it so much, or under utilizers, then why aren't you using it. You want to identify those two. We have some of those. I mean we've identified a couple of those on the underside and myself who is probably on the over side. What is going on with that. You just focus on that and say, how can we make it easier for you or is there something. Do you need a refresher, need reeducation, do you need me to show you what to do, and that kind of stuff. You have to identify those in order to be able to elicit change though.

\section{Receiving updates}

We have had the people from the HIE ... or where ever they are. They've been through, it seems like a couple of times since we started [the HIE]. Get some feedback and asking similar to what you are asking, what could you use it more. [Do you see any changes after they have talked with you?] It has been the same for me. I have not noticed any changes now.

We have had a couple of people come in and talk to us... Maybe once every six months or so. [Did they give you any in-services?] Yes. Awhile back. And whenever there is an update, they will let us know. 
I know there's some little things that change on [the HIE] and every once in awhile it will be down because they are upgrading something. But I hadn't really noticed anything dramatic. I know they got the discharge summaries on a couple of years ago-that was nice. It was a little bit delay in things, like if somebody within the last 24 to 48 hours that stuff may or may not be on there from the other hospitals.

\section{Contact with HIE personnel}

They [HIE haven't in awhile. They used to [come and tell us about our usage] and they asked about [the HIE] and they followed it. I was aware that they were following the usage and they did ask why some of it had fallen off. I told them about the patient population has changed slightly. I think I am fairly constant in my level. Some doctors don't use it. It is just varying, I guess, characteristics of the doctors as to whether they think they need it or not.

But our utilization is still running about $40 \%$. I would like to get it higher than that but before it was 7\%. [Are you one of the highest in the city?] Well, I wouldn't say that, I would say yes, but I don't know the numbers from the other facilities. I just know mine. I do know it has gone up pretty significantly. But yes, the utilization rates go up as the interface gets better. I have worked in the same hospitals before with problems with the interface ... they will ask their nurse or their PA, hey can you spend 15 or 20 minutes and try and login to this system for me while I go and see a patient and maybe I can get some information from [the HIE]. And that is where [the HIE] is a miserable failure ... They're constantly updating it, they're constantly coming and asking for feedback, and they're constantly saying how can we make this better for you guys. Their coming to the physicians as the primary users and end-users that goes a long way you know. They know the players, they know the medical directors, they know system medical directors, they know all the physicians. They'll actually come up and say, hey you know Dr X who is not a director, not a system director, Dr X how can we make this better for you? And then the next thing you know it will be just exactly like Dr X really wanted it to do. We'll really listen to input. I think having the amount of institutes that we have with a good education on the importance of it, showing a demo of how it could possibly work, has been another big key. When [HIE management] started rolling this out, he basically said listen, this is my vision, this is how I really think [the HIE] is going to go. And then developed a demo and people said well that really could work. And then to really get everybody's input, everybody's buy-in to it and then take it to the physicians and say here's our product. Here is what we are thinking about doing, is this something you would like to do and how can we make it better for you? And then not to rest on their laurels, after they get this integrated in, they said well it's great you know, we have this, everybody's in, reading the medical records into the system but now we are having problems with access so let's go back and rework it. So a constant streaming has been nice. Again, the big key is to have an independent group that is not related to one of the hospitals, that doesn't have any interest whatsoever in who does what and what does who, but at the same time that's what they want to do has been key to the survival of this particular project and the success of it. As I said, this is really a model for not only regionally, but nationally as far 
as how it works. Because it is seamless, it works great. [It sounds like you are one of their key players that they are coming to you?] I would think so. I mean ... [HIE

management] and I are on a first name basis so he calls me, asks me how things are going, and I don't think that is the case [in other facilities]. I actually worked at [other hospitals in the city] before ... So I have been there, done that. And they don't and a lot of that is they just don't have anybody that specifically works with [the HIE]. It's a little more difficult to find somebody who's at the head of the class. There is a lot more, they're just a lot more spread out, so they don't have anybody they can come to specifically, to move change. So subsequently, it doesn't get utilized. I think they're still on tokens.

I tell [the ER physicians] how to sign on, they use their user name, and they sign on. It's pretty much everything is there.

So far it has just been learn on my own.

Obviously, I didn't use [the HIE] for the first few months I think just because I didn't know it existed. Pretty much. I knew, I never heard anyone talk about how helpful it was.

\section{Communication between emergency physicians}

Our ER director has been around here forever, uses it quite abit and he was the one really that I heard talk about it the most. The other guys, I never hear them talk about it.

I went a few months here before I got it and I didn't really know how helpful[the HIE] was. I had heard almost nothing about it. There were a few nurses that had access so we would go to them if we needed to know. If this was a new left bundle, you would go ask this. The chest pain nurses all had access so you would say, would you pull up a read from the old EKG. I think nobody talked about it. It is probably one, if you only used it once a month it would be more cumbersome, but in fact, I use it so much that I, it is very fast around my workflows. Obviously, I didn't use it for the first few months I think just because I didn't know it existed. Pretty much. I never heard anyone talk about how helpful it was.

I don't really talk to the other guys about how much they use[the HIE]. I just know it's very helpful sometimes, extremely helpful sometimes.

Well, the labs, you can also if I can remember. I think when you click on the labs, if you can get I think there was an icon I am not using but it can give you the lab value.

Somebody comes in with a low blood count. What you can do is, I think it is tedious, but I think there is a place where you can get a graph or like what the patient's blood work has been in the last couple of months, like the last three years. Otherwise, you have to go to each visit and pull down lab and go to the next visit, and pull the lab, another visit pull the lab. I've not used the way you can find the range of the blood work for the last couple of visits, like somebody with a creatinine. You look at their creatinine values, is it going 
up or going down. I think it is there, if I can remember when they came to talk to us initially, I think but I have not used it.

\section{Interoperability}

It is a bit of a problem because you know its access to servers, or server only. So there is some really significant changes and differences.

I mean, most of this medical record that's the problem. You know you start, it looks good but that's a work around. You see glitches because it is wasting my time. I go to the room with the paper chart, I get my information come back, and have to document it [on the computer].

\section{System and software communication}

I know when we first gave information to [the HIE], it wasn't that we didn't want to give them information in the discharge summaries, it was that the computer language didn't talk to one another such that the information wasn't as easy to transmit as the labs and $x$ ray reports. So even though we wanted to, it was a huge deal to provide that information without major investments of the language so that each computer could talk to one another. Does that make sense? Maybe some of the difficulties with the other facilities, I don't know whether they have just opted not to do it or whether that they just don't talk. I know on our end I have talked to [Senior Administration] about it and was told that we wanted to but it was the wrong format to communicate to one another.

Well, I think everybody has had exposure to physician online entry or physician medical records and so everybody has their things they like and don't like. But it is, in my mind, kind of marrying a lot of the good ideas together into one system would have made a lot of sense. Just going through and figuring out, people like this part of the EMR over at [this facility], people like this part from [another facility] ... and seeing if there is a way to bring some of that usability into the system I think could have made it even better. What they did was fine for what it presents. I did not build it and I don't know what challenges they faced in getting the data coming from three separate systems and getting it to pass through into one system and then generate it in a visible form. I am sure there it was tremendous, all the patches they had to write to put that together. I don't know what their limitations were on that side so I'm just ignorant to that to be honest with you.

I don't know what it is, but sometimes I can sit down today and put a patient in and [the HIE] will say they didn't have a visit in our ER. I just saw them two days ago and it won't be there. I don't know if it is just not there because it didn't get put in right. I have no idea why sometimes there's a gap. I don't understand it. But sometimes we do get gaps, not often, not that it's noticeable or anything like that, but I just saw that patient and I wonder why information is not in there. 


\section{Standardization}

I mean the documentation ... you have some hospitals using [electronic] health medical records like this one here, and some other hospitals use paper charts. By the time, they scan that into the computer... No, it is not standardized.

I would like images, $x$-ray images and CT scan images if I can. Compare the two. If we do a CT scan on somebody, they would like to know that. CT scans, x-rays, EKGs, most of the discharge summaries are there. Some hospitals don't put everything in there so you can't look it up that way. No [the HIE is not standardized]. What's in it is standardized. What each hospital, the way each hospital dictates its medical records is not standardized. We don't dictate our notes, so you can't see an ER note from somewhere else. We don't dictate it in the computer. Our notes are written. [Emergency departments with electronic documentation] dictate their notes, so computer and stuff is in the computer.

You know the only thing that is not standardized is clinic visits. And that is a broader problem with them because they have so many different clinics and they handle their clinic visits completely different. We'll get some of that, especially those that do an electronic record that are easy to transcribe. But for instance paper, we are not able to get paper in. I will give you a classic example like ourselves. [Another hospital] does not get my ER note. They see that we did labs, see that we did $x$-rays, and if they're admitted, they will get a $H \& P$ and a discharge summary. Anything they want as far as in-house, but they will never see my ER note because my ER note is paper.

I think what you see are a lot of varying levels of completeness from people dictating their discharge summaries. I've seen some, and there's some physicians around the city that are notorious that they'll dictate a two-paragraph discharge summary for a twoweek hospital stay. Well how useful is that. They'll say see H\&P for the details. I think what they do in the HIE just presenting them is fine.

It varies by institution as to what information is in there. [One hospital] has H\&Ps in there but their discharge summaries maybe 48-72 hours delayed getting in there. [Another hospital], they have radiology and lab. I don't think they have any discharge summaries. [Another] has discharge summaries, $H \& P s$, and reports, lab reports. You don't have ER records at other facilities, so it is variable from institution to institution what information is available.

For instance, from [names hospital with paper documentation in the ER] we can't pull up their H\&P from ER. But they can't pull up ours either. They can pull up if they get admitted or discharged or H\&P and they're discharged because they are dictated and transcribed in the medical record and then we can pull it. They are able to pull up text, just not pictures. But yes, absolutely. I get excited when I see a patient was to [hospital with EHR] because I know I will have a note from the ER doctor that will tell me exactly what was going on. 


\section{Information needs}

Also another thing that would be very useful is scanned in EKGs, because we get a interpretation, but it's not the same as, OK just looks identical versus, yea, they both had non-specific $T$ wave changes but now there is a clear pattern to them where they wasn't before.

If they were at outpatients, in clinic, they were in the ER. They specifically designate exactly where they were. But again there are some issues. They don't scan the documents into the [HIE] and then be able to pull it up ...I know it is a lot easier for them to do type than it is do pictures and I think that's a lot of it. Basically, a scan would have to be a picture document and they would have to change a lot of the software. Now the upgrade version may allow them to do that, but currently, no.

It would be nice to be able to graph it to see trends and data [in the HIE], but that takes another level of integration that we currently don't have. That's basically where everybody is on the same system, which we're not. I don't think it is going to happen because in order to be able to have those data points and be able to track, you have to have a software system that's able to track those data points individually to be able to make the graph. We don't have the capabilities of doing that right now. It is all individual, basically screen shots of the lab values.

One of the pitfalls is you don't always get discharge summaries. You don't always get information. The main things is the labs and radiology results, and if there's a discharge summary that's a bonus.

If there were outpatient providers would be very helpful. There is no outpatient records usually so if someone has their chemotherapy doctor that they see every week I have got no record of that unless they have actually have been admitted to the hospital. So consultations, outpatient consultations are often very helpful.

I don't need the whole record. I don't want the whole record. Because again more clicks more time. We have lab, radiology, discharge summaries, $H \& P$ s, it's perfect. And the ER doctor perspective. Now the future, they may want to look at different variation based on the user and who it is. In other words, they may have a different server for ER doctors and have all the information for an internist. An internist may want to see what did that ERCP that was done while the patient was in the hospital last month. I'm not really interested in that from the ER standpoint but he would be from the standpoint of managing that patient. In the future, they may want to look at different user interfaces, different values as far as access to the medical records, and different amounts of information based on the user interface. That may be another future possibility but from our standpoint who knows, I think we're $O K$.

I think we do [get enough information], the only one thing that I wish we had a little bit more access to sometimes especially in ERs, we don't list all their medications or something like that. We may leave some things out but other than that, I think it would 
probably be good if we could get access to some of their clinic documentation. But even with the ERs, if you put it all together, if they have been to another emergency room, you can kind of piece it together-what their past medical history without their clinic information.

\section{Connectivity and equipment}

Usability has a number of different factors to it. Number one is access, meaning that I have to actually have the device and I have to have access to the device, which has been kind of a challenge here. So, that's one of the biggest things. If you have multiple different users, you have to have multiple different devices. So access is the first part of usability. You have to have access to the device ... Well, access for us is just the sheer volume of users. We have a problem with the number of computers that we have and the way that we designed the emergency departments for the past, [which] has not been conducive to having access points for all the different users. We have a brand new emergency department ... an expansion. We're having to go back and redo everything because we just didn't think about the access... Not enough computers. Doesn't make easy usability for the physicians to be able to use it. Ideally, we would love for our physicians to be able to document at the bedside when we go to a completely EHR. That includes from being able to pull up medical records and say, oh, Mrs. Smith I see you that you were at [another hospital] and you had appendicitis and it looks like you had surgery. Well, we don't currently have the capability to do that. We have the wiring, but again, and the way it was designed we even have where the physician would have his back turned to the patient the entire time. That's not what you want. You don't want to see your physician's back so there are some challenges involved in implementing the EHR into the healthcare.

Some computers are just slower than others. And so it's all about speed.

We've looked at all those. It's a possibility. There is ... the smaller portable devices don't work just because of the possibility of medical record errors, meaning your cell phones, smart phones they don't. Now the iPad or something like that has a potential because of the ability to number one, write and transcribe into the medical record, and number two, just the font size is big enough to do it. It also has a pretty good firewall and is pretty easy integrated. MacIntosh and Apple have done a fairly good job as far as that. Eventually, I think we will probably have to do something. Now I have used laptops in EHRs with touch screens and pens, very cumbersome. You give up a lot on the portability. It's great to have the portability. The interface is not quite there so you lose on the productivity. You'll see very, very few physicians right now that have the capability of using laptops use them. Because again, it's difficult, portability is good but the interface is difficult. There's not an ideal device... [and when you are with the patient?] And that's where I think portable devices have a much better integration possibility in the future than we do now ... having the access at your fingertips. That's where portable devices really have their role as far as the HIEs. Because of having that access at your fingertips and not necessarily just the documentation, but more of the 
access to the information. But it has to be the right portable device. If it's too small, it is not going to help you. If it's too cumbersome, it's not going to help you. It's got to be perfect and that is why I think the iPad or something similar to that will probably be where you go. Now I have used tablet PCs before and they're flimsy. They have a tendency to be dropped so you have to have something cheap too.

[Portables that you actually take into the room, would that be beneficial for you?] I don't think so. Why, because with the patient you talk to them and examine them and then everything else you can come back to your room and do it. But, there are computers and sometimes patients ask you for a result that you don't remember off the top of your head, you can look it up and show it to them. That is OK. But I don't think I need a computer every time to go in to do this.

But [another hospital] has computers but we still do not go with the computer in the hand. They do have a hand-held there.

Laptops are always a little slower.

[Are there enough computers?] Well, not really. You have to share and sometimes you have to wait until someone gets off the computer so you can get on. I mean it's not that big, because usually there is enough work for me to do that if someone, or two or three people, are on the computer and there's not a screen access, I will do something else and come back. I'll sign on later... I work around that and then I'll come back and they're off the computer and I can get on.

[Are there enough computers?] Yes, there are three, it is good enough. We are two people usually during the day. We have enough devices.

A lot of time you may just have two computers. Like here there is more. Some hospitals have two computers, which the doctors can access. Well, you have a 30-bed [emergency] hospital, so you have to run around, going back in, sit down, and record it.

\section{Hybrid work environments}

I think it's one of those things, where I think other facilities doing [electronic ER documentation] so I can see their records benefits me ... I am not a fan of [the EHR] but I understand their needs to be some reciprocity there but just from a purely selfless standpoint, I think it is going to take longer. You end up with typically, the EMR produces pages and pages of patient records that don't convey actual information. A lot of our outlying facilities use T-sheets, which don't really convey information either, but they do it in a more efficient form because there is only two pages of none information versus eight or nine. But you will have an EMR that basically just downloads all of the interactions that took place. You have six pages of patient pillows fluffed, and not I did this because, or here are the labs in a conveniently located area. Instead its kinda flipping through, got a BNP here, I missed it, troponin here etcetera. I haven't actually 
seen a good EMR transfer worthy document. I think dictating is kind of the most efficient way and I guess if you did a text to speech. I understand why they don't do that because it is much easier to bill off the T-sheet and it is also very easy to bill off the EMR. EMR does a fantastic job of generating your level five documentation that's got the review systems and you don't miss anything, because you can't miss anything, you have to check a box somewhere on there. But in terms of is it good for billing depending on if its prompted OK for legalize. You pull up the chest template and its going to prompt you about do you think it is a PE by asking you about the welsh criteria etcetera, or you have to click off no pluritic chest pain, no family history of $x$. It doesn't really convey patient information.

\section{Theme 4: Recognizing the Benefits and Success Factors}

I'm a big fan of [the HIE]. I think that it will make for more efficient use of the health care system. I think it has a tremendous capability to improve patient care and prevent patient injury, like from repeated radiation or multiple frivolous work-ups that are just repeated over and over. I think secondarily, but importantly, it has the tremendous potential for cost savings. I mean instead of everybody operating in the silo you have connectivity between the different facilities. If someone just had something done for this same problem, and they are unhappy with it, and so they come over here three days later. I can access that information and say normally I would do this work-up but it was already just done and so they need to follow up with their primary care doctor or go onto the specialist. I mean that has potentially saved thousands of dollars in patient visits just because you're not reinventing the wheel. So I think improve patient safety, make things more efficient and improve patient care, and cost savings. I mean there are three significant pillars to build on right there ... Your overall capture of those three things at implementation. I think you would have a better system and that then it is more efficient, it is cheaper.

\section{Avoiding Duplicative Testing and Unnecessary Ordering}

Saving on radiation to the patient. The benefits are humongous.

But if I saw that he was in the hospital two days ago for the same thing and they did this, this, this and that, then I don't need to repeat it. I don't need to radiate you anymore and cause more radiation.

And as in a lot of tests if I think that the patient doesn't need it and then I can find confirmation that they have had it done elsewhere, then yes [I don't need to repeat]. You can't get the ER note if they have just been to the ER. Yes, so what I am saying is I don't order a CT on every single patient. It just is kind of a gut feeling of if you've got somebody, you have belly pain for a long time and you have had three CTs in the last month, and you can pull them up and see that they were all $O K$, then you're probably not, unless something is really a drastic change. It is just kind of every case is different but the 
more information, if you had a full note, and exam and all that yea, that would be a lot more helpful.

I think the access to data of recent workups is very helpful. I think that is a tremendous advantage that [the HIE] provides because you pulled it up and will say, they've had five CT scans this year for this problem ... I don't need the CT here.

[Do you see a decrease in the number of CTs people are getting?] You know, no. I don't think so. The problem is we get sued so much. I'm more worried, this is probably not a good way to put it, but reality is reality. I'm more worried about me getting sued than the radiation I am giving that patient. I hate, that's not a good thing you know.

[Do notice a decrease in some of your tests ordered?] Yes. [Do you think much of a drop?] Hum, no. I don't know overall probably not. I mean I guess in certain cases ... it would just depend on how I felt about the patient whether I would reduce certain things or not. But [the HIE] probably helps some I think.

I think [the HIE] would seldom keep you from doing blood work unless they had just had it two days before.

\section{Efficiency in Patient Flow}

[And what makes you go to the HIE to use it?] Only if it is going to help me to interpret studies that I've already received. Most often, it is that. So if I come back with an abnormal test, I want to know if their test has been abnormal somewhere else that would be very helpful. Or, if I am trying to decide whether to undergo another imaging. If you have somebody with belly pain and you're saying you know this belly pain seems concerning, has it been imaged recently and if you look and see that they have had a CT scan within the last few months this is very helpful sometimes. So if I am trying to talk myself out of performing some diagnostic test that is going to keep them in my ER another hour or two, I can save the entire through-put through the ER if I know that they have had testing recently.

I think we save money for the department ... By saving one less CT means less time and you see one more extra patient.

I am looking for most recent admissions, ER work-ups, significant labs, or imaging CT scans, and things of that nature so I would not have to repeat those.

In my decisions as an Emergency Provider, I think you're treating the patient's very acute illness. Often it is very specific patients, the belly pain the CT is not going to change often. Often doesn't change. It can change disposition of the patient as far as where they go. It may save some time in the ER more than change what you're going to do. 


\section{Conundrum of Protocols}

For the most part protocols are good. I like protocols. Some docs don't like them but I do, I like them. I like having stuff already waiting on me that they have already ordered.

Lab tests usually are ordered prior to me seeing the patient. So I think there are sometimes I would not have ordered tests because they just had normal labs yesterday and they haven't been vomiting or something that would make me think that they're labs would have changed in the last 24 hours. [How do the tests get ordered?] The nurses have protocols. For this patient with chest pain, these labs get ordered, which helps when we have 20 in the waiting room and so by the time they have waited four hours hey, almost everything is back.

\section{Fiscal Benefits}

\section{Reducing diagnostics}

[You are saving on some of the radiation for patients?] I think at least for patients one or two, at least three to four a month maybe. Which is to save something better than nothing.

I would say [the HIE] maybe avoids one cross sectional imaging study every couple of days ... we don't see a whole lot of people that were crossing hospital systems, kind of with the same acute problem, we do sometimes, but it's maybe two or three a shift versus 10 or 12.

I would say it maybe avoids one cross sectional imaging study every couple of days.

[Do you ever see a decrease in your CT scan ordering?] Do I, because of the use of the HIE, sometimes. And as in a lot of tests if I think that the patient doesn't need it and then I can find confirmation that they have had it done elsewhere, then yes. [Do you see much of a decrease in a month's time? ] Several I would say.

\section{Obtaining medical records}

We used to try get medical records from other facilities faxed in the middle of the night. A lot of times that is hard because medical records close at five and you have no access to any patient data at all, you don't know.

They come to a different facility and you have no access to that information other than what we traditionally use, which is time intensive. Your unit coordinator has got to fax the information over to the medical records and they pull medical records and fax it all 
back to you. It requires two or three people working unilaterally and just specifically on that one problem and it is very inefficient.

\section{Reviewing records}

There is a little blow up because someone says that is my patient ... I'll go pull a record and the patient who said this was their primary so it falls on the hospitalists because they will be criticized for that. It also falls on the emerg department because they will say, well their primary care doctor was this one and you admitted them to the hospitalist. I have to pull the chart and read through it. Our EMR is kind of cumbersome. It is hard to read to find out what happened and usually you can run it down. A lot of that would be alleviated by just being able to pull up [the HIE] and see if they have seen a primary care doctor, end of story.

Just admit them to whoever is on call ... And that person may not know the patient and they will send them home on medication that they are already on. You end up duplicating. It is like this. The system needs a lot of tightening.

\section{Lost productivity with EHRs and HIEs}

Most of the time when you're actually on a computer you spend about 50\% of your time on a computer, $25 \%$ of the time we spend doing other stuff, and $25 \%$ of the time we spend with your patients. It's a big change from when you have papered systems; actually, that goes up significantly more time with the patients. But your productivity is kind of questionable whether or not you are actually getting what the patient really needs. There's pluses and minuses to [EHRs]. We're in the process right now of just about to implement a brand-new EHR in here. We're going to stop the paper and go to $100 \%$ EHR. With Medicare, CMS indicators and guidelines for 2011, which means that you have to have it in, we will do it, and it will be combined. Not a whole lot of people are happy about it though, because you lose about $30 \%$ of their productivity from the physicians. We have gone ahead and in anticipation of that loss of $30 \%$ of productivity, we're looking at other ways to help out with working with the documentation. We have hired scribes. Scribes are basically, all they do is just go around with us and do all the documentation. Eventually, they will be doing all the computer documentation or at least most of it. That will help us recapture that $30 \%$ of lost productivity when we go to this. It gets better every year. It starts at $30 \%$ and then down the road, usually in a couple of years, it's about $10 \%$.

Like going to exit care in our automated discharge summary, used to take 10 minutes per patient, so a lot of the scribe's benefit especially at first was the fact that they could do the exit care for us so we basically had to hire someone to do what we used to do. They do other valuable things as well, but to give us back the productivity we lost by going to an electronic system. Now it is useful to be able pull up, OK this is what the patient got 
discharged with but those also are available in a form there about as easy to obtain do the scanned in records.

\section{Theme 5: Current View}

Well we're fortunate in that we have a pharmacist in the ER ... We're very fortunate. Now we don't have them at night, so you get the nurse to call Walgreens or any other pharmacy that the patient tells you they go to. It's pain staking, it's time consuming.

No, we don't have pharmacists in the emerg department. If they have a previous visit, they'll pull-up the medications from their previous visit or the nurse will go through. What usually happens here is a nurse will go through their medication list and they will enter it manually into the system.

You don't know [what medications the patient is taking]. There is no way, I mean if you can't get that, that happens a fair amount. I'm on a heart pill, I'm on a blood pressure pill, and I'm on a sugar pill and everybody is on a little pill for something. But I mean I guess if you had their pharmacy you may call and see ... and sometimes [the patient says] I got the list at home if you give me a minute I'll call and get it from my daughter's at home or something. But otherwise you just say, you don't know and so you have to say you don't know and you just have to go with what the patient has given you.

I have never seen ER physician notes. I have seen procedure notes, discharge summaries, sometime H\&Ps, but that's mostly what I get. I don't think ER notes.

[The ER physician note] just doesn't address immediate problems. No matter what is in the note, if the guy has done studies and that shows one thing and the guy comes in with a different problem or increasing problem, it's good if they have done an x-ray or they have done a radiology study or labs. But to get his note is not really going to change much usually.

I would love to see the ER physician notes. Definitely that.

[Do you see some validity in that, having an ER module?] No, I don't. It wouldn't make any difference to me. I mean all the information I think it would be duplicitous to have it in different modules. That all the information that you need is right there so whoever is looking at it doesn't matter ... And I would think it would be unnecessary to duplicate information or to have two separate systems that would be management and money waste in order to do that in my opinion.

I can see that [an ER module] would be helpful. Right now, there is not much in there usually.

When we're in this health care, the economy, and trying to decrease the usability of ERs and stuff, I can't see any negatives at all. I really can't. It's all confidential. The only 
people that are using it are health care providers, and mid-level providers. Nurses and stuff, we don't allow them unless they are a nurse practitioner to have access to other patients' information. So I don't see any negatives. If it grows from here I can only see a positive.

[The HIE] doesn't apply to every patient and that's why I don't use it on every patient. And I think the more modalities you have available to you the better off you are. The more resources you got available to make decisions, the more likely you are to make wise ones. But you don't use every modality with every patient that you see.

Yes, that it would be more user friendly and I think the better and the more I would I would use it.

[The HIE] is a great model for what we anticipate the national HIE to be. We have people come in and look at why is it a success here because it has been a failure, a miserable failure in so many different markets. I just said I think overall I am a big fan. There is certainly some drawbacks, but overall it is in the best interest of your patient. When I started practicing, I could never even image that we could get to this point. I started practicing and I was running down to the medical records room all the time trying to find out, looking through charts, and spending hours and hours and hours going this patient had an MRI that was done whatever. Or, a CT scan that was done four years ago, that showed me something. To have that availability at your fingertips is exciting.

You know there is one issue that it always kind of bothers me a little bit is I think there is an expectation by the patient of confidentiality. I know when they come into every facility they always sign a disclaimer saying you share your medical information. In a sense I think it is betraying to the patient a little bit ... if it is a complicated patient they will go somewhere else to get a second opinion- an independent opinion. They don't expect you to take the other guys opinion and walk with it. They want you sometimes. They'll come in because they're expecting a fresh slate, and then say hey what's going on here.

Because some people have complicated hard problems ... When they have them sign the papers now, they have them sign specifically for HIE sharing, if I'm not mistaken.

\section{Theme 6: Non-Use or Reduced-Use of the HIE}

\section{Failing to Provide a User-Friendly HIE Coupled with Time Constraints}

[You have said the HIE is a useful tool, what would be the reason ER physicians are not using it?] One, some people are not aware of it. You would be surprised, some doctors, you tell them you can use it, oh, how can I can use that? So one I think you guys need to be a little more out there educating people. Two, you know this having to go close this window, go open up another window. And, another one is just bad habits. Some people are just used to doing it checking out records, or just used to the old fashion way to call somebody, or they don't care to duplicate tests anyway, I don't get it here, I am just going to do the tests. 
If [the HIE] was integrated into your EMR process then that would make it a lot more seamless and would probably increase its use.

[Why are physicians not using the HIE?] Mostly I think because it is an extra step. And it's actually possibly multifactoral, it's an extra step ... You have to put in the patient's name, you have to look them up, and one night every patient is going to trigger that you need to do that. Like the patient that seems like, I had a cough and I haven't seen anyone for it. OK, that's kind of more people that we are concerned about drug diversion or people present with kind of, oh yea, I have gone to [another hospital] every three weeks for the last eight years and this is my first time [here at this hospital]. You are just trying to get up to speed and a lot of times, I think that we may feel that the history that we have gotten is sufficient to be able to be able to make a quick, does this patient need to be admitted or not. Then let someone else do the grunt work of getting the nuts and bolts of it. Especially when it gets busy, there can be sometimes more triage than fine tuning treatment.

I never talk to anybody specifically about [the HIE] but I would assume it is probably the same reason why I don't use it as much as some others. It is a little bit cumbersome, it is time consuming, and that maybe is the same reason why some don't use it as much as others do. I mean I have never talked to anyone specifically but I think the more userfriendly the more I would use it.

You go back and try to dig for the chart. That's why a lot of doctors don't even bother using [the HIE] because it is too many patients to see. I know some physicians who seldom use it.

If I am extremely busy then I don't have time to do [the HIE]. Usually what will happen is I will wait a couple of minutes until it cools down and then I can try and look at something but that could be pretty much a cause. Some hospitals have single coverage and you are very busy running around doing a lot of stuff and you don't have [time]. Some people might have the access that takes a lot longer than other hospitals, I don't know how it is in each hospital. They might not have the time to go look it up.

There are all sorts of doctors you know, some want to crank patients out at a high rate of speed, and [the HIE] would slow them down.

\section{Medical Legal Concerns}

[Law suites] are just a problem that we just have to deal with, I guess there is no way. They show if there is a cap, like states that have had some caps, the malpractice rates are down and it maybe decreases some but it is still there. These suits are such a hassle, and just even having to go and do the deposition and go through all the stuff. Even though it may not exceed my policy limits, I have to worry about them coming at my personal belongings. It is such mental [strain], it messes with your mind. I don't have an answer to 
that because there are so many things. The problem is too big, that is why we're in medicine the way it is because nobody knows the solution.

There are two things nobody will say. One, like I said, it is the difficulty accessing [the HIE]. The other one is just bad habits, and some people, it is just medical legal. What if somebody had missed something. What if, you know what I mean. Say someone goes to the hospital. You get an x-ray or CT report and the patient comes here. Some guys say, what if they missed something there. It is medical legal but nobody will say that to you.

Now this may be another reason why other physicians don't use it because we have a lot of patients, drug seekers, that come through the emergency room and if you know that they have been to [another facility] ... and just got 12 Percocet ... because patients will say, no they didn't give me anything for pain.

\section{Biasing Clinical Decisions}

The only thing that I can think of why a physician would not use [the HIE] is I think maybe they don't want to bias. Like say for instance, two days ago, you were seen at another facility for the same compliant and they did a workup and they didn't find anything. Would you not repeat the same test again based on another? Maybe that's why they wouldn't. You see what I'm saying? And you're thinking they have just been to another ER and they have this, this and this done. Why are they here so maybe they feel that might bias their opinion about that patient if they look and see if they've been to another facility recently.

I very rarely know anything about a patient before I go in the room to see them ... Because that is not how I learned to practice medicine. And so the information's good and the technology is excellent, but on the other hand, I think it has made lots of the young physicians' minds very lazy in their approach and their ability to analyze the situation because they already know before they see the patients. So when they can't figure something out they have, their brain hasn't been exercised enough to know how to figure it out if they don't have the information in front of them and sooner or later you're going to run out of data or not have access to data. And so what do you do then? How do you make decisions then? Well, you probably can get by but that's going to be a weak area in your ability to analyze and care for patients.

\section{Lacking HIE Education}

Whatever means you guys have to educate doctors, usefulness of this system. Like I said, it is a difficult habit, it's a culture. Some people just get bogged down and just want to see patients. They don't want to be bothered with accesses.

[Why would you say other physicians aren't using the HIE?] I think it's an education issue. I really do ... We don't have a flashing button that says, please access me, look at 
all the visits from all the different hospitals. I think a lot of it is still an education issue. It's getting it back out there. You know we've actually gone through that trying to figure it out. There are a couple of users and they seem to be older and they just keep forgetting. They keep forgetting they have that capability of doing it more than anything else ... just continuing to reeducate them and continuing to keep a positive attitude towards it has helped a little bit. But you know, it's still an old dog new tricks are hard.

\section{Changing the Culture}

Some doctors don't use it. It is just varying, I guess, characteristics of the doctors as to whether they think they need it or not. The doctors that use it use it and the ones that don't, don't.

Maybe good to have it but he is not going to change what he is doing here. And I say that within the realm of the specialty of emergency medicine that a lot of data that other physicians need to provide ongoing care is not really necessary for us to make decisions about. And you can leave that information retrieval to the person that is going to actually use it, where it is going to impact the patient in the long term whereas what we are doing is just really looking at what we are doing right now. And if you put the blinders on and you stay within the realm of the emergency medicine, a lot of this information retrieval belongs in the lap of somebody else. And not us.

\section{Competition}

I can see [the HIE] would make patients less loyal to a particular hospital if they knew that their records could be easily accessed somewhere else. It is certainly the right thing to do. It may. I can see from a patient's perspective if they knew they could go to any hospital in town and have their records accessed, I can absolutely see that. Most patients are loyal to providers ... They say my doctor comes here and my doctor doesn't go there so I don't know that it would actually impact many patient decisions, but certainly the potential is there.

\section{Lost Revenue}

I can tell you in certain areas that they might not want to use [the HIE] because they couldn't really care what they are going to do, they are just going to repeat it whatever. In some areas then they use it more. It is an insurance issue. People with insurance get more tests because of billings whereas the [names hospital] is mostly the uninsured and therefore not a benefit to giving extra tests.

Because you gotta do what is right for the patient and if it's ordering everything then go for it and if it's not then don't. I haven't heard anyone in administration ever say you guys need to order more tests. I know there are emergency departments where that 
definitely does happen, where there's a we've got a good payor mix and you need to maximize your revenue. If you have any question then admit them etcetera. But that's not how it works here.

\section{Lacking HIE Champions}

Initially, I think we were kind of slow getting started using [the HIE], we weren't using it as much. There was one physician that was really the champion. She used it all the time. It was like she was this mighty HIE person, but now all of us. I think we have got to know it and love it.

[Why are HIE personnel not helping other facilities with your successes?] That's a good question, I don't' know. I really don't' know the answer to that. I think it is something they should. I don't know if it's just the fact that again they don't have a strong champion. I don't know if it's an access issue, it's a communication issue between providers. I know that several different facilities, you have different champions at different facilities and therefore you have some people who may have different ideas. Some it is a little more difficult to get the word out in some other facilities as far as what they are trying to accomplish. So that may be a lot of it.

\section{Failure to Grant Access Privileges}

[When the nurses pull up the information, do they tell you what's in [the HIE] or how do you get it?] They just kind of let me look I guess over their shoulder.

There is a doctor working there, he was in Iraq and he came back, and we told him about it, but he didn't know about it. 


\section{VITA}

Shirley A. Thorn, born in 1961, received a Master in Nursing with a major in Nursing Administration from the University of Manitoba, Canada and a Master of Administration degree with a major in Public Policy from the University of Regina, Canada. She has experience in senior administration, government, education, program management, academia, and research. She has spent a number of years in various hospital settings, including pediatric and adult settings in both Canada and the United States. Her recent focus of study and research is healthcare informatics, nursing leadership, and health policy. She is a member of numerous nursing organizations, university committees, and the State of TN clinical workgroup for health information exchange development. She is currently Faculty at the University of Memphis. Shirley successfully defended her dissertation March 9, 2011 to fulfill the requirements for the degree of Doctor of Philosophy, with a major in nursing. 